\title{
FUNCTIONAL CALCULUS IN FINITE TYPE I VON NEUMANN ALGEBRAS
}

\author{
PIOTR NIEMIEC
}

\begin{abstract}
A certain class of matrix-valued Borel matrix functions is introduced and it is shown that all functions of that class naturally operate on any operator $T$ in a finite type I von Neumann algebra $\mathcal{M}$ in a way such that uniformly bounded sequences $f_{1}, f_{2}, \ldots$ of functions that converge pointwise to 0 transform into sequences $f_{1}[T], f_{2}[T], \ldots$ of operators in $\mathcal{M}$ that converge to 0 in the *-strong operator topology. It is also demonstrated that the double *-commutant of any such operator $T$ which acts on a separable Hilbert space coincides with the set of all operators of the form $f[T]$ where $f$ runs over all function from the aforementioned class. Some conclusions concerning so-called operator-spectra of such operators are drawn and a new variation of the spectral theorem for them is formulated.
\end{abstract}

\section{INTRODUCTION}

A classical (continuous or Borel) functional calculus for normal operators is a powerful tool in operator theory (let us mention only a single deep application, namely, the functional calculus for selfadjoint operators is involved in the most common proof of Kaplansky's density theorem for von Neumann algebras; see 28, Theorem 5.3.5 in [25], Theorem 1.9.1 in [48] or Theorem II.4.8 in [50]). On the one hand, this calculus has intuitive properties (such as $(g \circ f)[N]=g[f[N]]$ where $N$ is a normal operator and $f, g: \mathbb{C} \rightarrow \mathbb{C}$ are two Borel functions) and is fairly flexible, and on the other hand, is, in a sense, as wide as possible and sufficient enough. Indeed, if $N$ is a bounded normal operator on a separable Hilbert space, then the set of all operators of the form $f[N]$ where $f$ runs over all bounded Borel functions $f: \mathbb{C} \rightarrow \mathbb{C}$ coincides with the double commutant of $N$ (thanks to the Fuglede theorem and Theorem 8.10 in Chapter IX of [8]). The aim of this paper is to generalize the concept of functional calculus to a much wider class of operators (and their tuples) - those which belong to (or are affiliated with) finite type I von Neumann algebras. Typical examples of such operators are (finite square) block matrices of commuting normal operators, and direct sums of such block matrices. To formulate our main result, we need to introduce necessary notions and fix the notation.

For any $n>0$, let $\mathscr{M}_{n}$ and $\mathscr{U}_{n}$ denote, respectively, the $C^{*}$-algebra of all complex $n \times n$ matrices and its unitary group. Further, let $\mathscr{M}$ stand for the topological disjoint union $\bigsqcup_{n=1}^{\infty} \mathscr{M}_{n}$ of the spaces $\mathscr{M}_{n} . \mathscr{M}_{\text {is a }}$ Polish (that is, separable completely metrizable) space. For any $X \in \mathscr{M}_{n}$ we put $d(X) \stackrel{\text { def }}{=} n$. Further, for each $U \in \mathscr{U}_{n}$, the matrix $U . X$ is defined as $U X U^{-1}$. Finally, if, in addition, $Y \in \mathscr{M}_{k}$,

2010 Mathematics Subject Classification. Primary 47A60; Secondary 47C15.

Key words and phrases. Finite type I von Neumann algebra; operator affiliated with a von Neumann algebra; matrix-valued matrix function; double *-commutant.

The author gratefully acknowledges the assistance of the Polish Ministry of Sciences and Higher Education grant NN201 546438 for the years 2010-2013. 
$X \oplus Y$ denotes a block matrix $\left(\begin{array}{cc}X & 0 \\ 0 & Y\end{array}\right) \in \mathscr{M}_{n+k}$. Additionally, $\|X\|$ stands for the operator norm of $X$.

A function $f: \mathscr{M} \rightarrow \mathscr{M}$ is said to be compatible if

- $d(f(X))=d(X)$ for each $X \in \mathscr{M}$;

- $f(U . X)=U . f(X)$ for any $X \in \mathscr{M}$ and $U \in \mathscr{U}_{d(X)}$;

- $f(X \oplus Y)=f(X) \oplus f(Y)$ for all $X, Y \in \mathscr{M}$.

The function $f$ is locally bounded if for any positive real constant $R$,

$$
\sup \{\|f(X)\|: X \in \mathscr{B}(R)\}<\infty
$$

where $\mathscr{B}(R) \stackrel{\text { def }}{=}\{X \in \mathscr{M}:\|X\| \leqslant R\}$. Polynomials in two noncommuting variables $X$ and $X^{*}$ are classical examples of locally bounded compatible functions.

Finally, let $\mathscr{F}_{1,1}^{\text {loc }}$ denote the family of all locally bounded compatible Borel functions $f: \mathscr{M} \rightarrow \mathscr{M}$. It is easy to see that $\mathscr{F}_{1,1}^{\text {loc }}$ is a unital $*$-algebra when all algebraic operations are defined pointwise.

The main result of the paper for single operators reads as follows. (Below id denotes the identity map on $\mathscr{M}$ and $\mathcal{B}(\mathcal{H})$ is the $C^{*}$-algebra of all bounded linear operators on a Hilbert space $\mathcal{H}$.)

1.1. Theorem. Let $T$ be an operator in a finite type I von Neumann algebra $\mathcal{M}$ that acts on a Hilbert space $\mathcal{H}$. There exists a unique unital $*$-homomorphism $\mathscr{F}_{1,1}^{\text {loc }} \ni f \mapsto f[T] \in \mathcal{B}(\mathcal{H})$ such that $\mathrm{id}[T]=T$ and

(bc) whenever $f_{n} \in \mathscr{F}_{1,1}^{\text {loc }}$ are uniformly bounded on each of the sets $\mathscr{B}(R)$ and converge pointwise to $f \in \mathscr{F}_{1,1}^{\text {loc }}$, then $f_{n}[T]$ converge to $f[T]$ in the *-strong topology of $\mathcal{B}(\mathcal{H})$.

Moreover, $f[T] \in \mathcal{M}$ and $(g \circ f)[T]=g[f[T]]$ for any $f, g \in \mathscr{F}_{1,1}^{\text {loc }}$.

If, in addition, $\mathcal{H}$ is separable, then the set $\left\{f[T]: f \in \mathscr{F}_{1,1}^{\text {loc }}\right\}$ coincides with the smallest von Neumann algebra on $\mathcal{H}$ that contains $T$.

In Theorem 3.1 we shall prove a counterpart of Theorem 1.1 for finite tuples of operators affiliated with a (common) finite type I von Neumann algebra.

As mentioned above, Theorem 1.1 applies to operators that are direct sums of finite block matrices of commuting operators (and, up to unitary equivalence, only to such operators). This result enables defining matrix-valued spectra of operators generating finite type I von Neumann algebras in a new and transparent way. This shall be done in Section 4. For other results related with the concept of an operatorvalued spectrum, the reader is referred to, e.g., [15], 21, 22, 29] and [32]. It turns out that the (classical) functional calculus for normal operators is a special case of the calculus introduced in Theorem 1.1. So, our concept is more general, and as flexible and wide as the former.

Compatible functions, defined above, resemble (but differ from) $n c$ functions studied in [27]. Instead of preservig unitary actions, nc functions have to respect (simultaneous) similarities. So, each nc function is compatible, but not conversely. Although the concepts of compatible and nc functions are similar, both the works ([27] and the present) are of idependent interest (and none of them inspired the other). The notion of a compatible function almost coincides with the notion of a decomposable function, introduced in [5] and studied in more detail by Hadwin [23] (see also $\S 5$ in [24]) who called the action of decomposable functions on operators a functional calculus. The main difference between our approach and Hadwin's (beside his restriction to separable spaces and ours to finite type I von Neumann algebras) is that decomposable functions are, by their very definition, defined on the algebra of all bounded operators on a separable Hilbert space and the scope of 
their operation (that is, the class of their operands) is not extended to more general mathematical objects, which is in contrast to the classical functional calculus for normal operators where the functions that take part in this calculus are scalar and scalar-valued, and the way they operate on scalars is extended to the class of all normal operators (with respective spectra). This is exactly the spirit of our approach. If we agree that matrices are 'simple objects' (in comparison to linear operators acting in infinite-dimensional spaces), the conclusion of Theorem 1.1 says that the scope of operation of (matrix-valued matrix) compatible functions may be extended, in a very reasonable and intuitive way, to the class of all operators that belong to (or generate) finite type I von Neumann algebras. Even more, decomposable functions have almost purely theoretical meaning, because they are hard to construct (apart from polynomials in two noncommuting variables $X$ and $X^{*}$ and holomorphic functions), whereas compatible functions, being merely Borel, are easy to do so (of course, they need to satisfy some additional axioms, but - as shown in Section 2 - there is a natural one-to-one correspondence, which preserves boundedness and pointwise convergence of sequences of functions, between all compatible functions and all $\mathscr{M}$-valued Borel functions defined on a certain Borel subset of $\mathscr{M}$ that preserve the degree of matrices). As decomposable functions are defined as operator-valued operator functions, there is nothing challenging in studying issues similar to condition (bc) (formulated in Theorem 1.1) for them. And it was (bc) that gave foundations for our investigations. (Actually, when we were preparing the material of the present paper, we were not aware of Hadwin's results. We learnt about them only at the final stage of the work.)

Operators that generate finite type I von Neumann algebras have been widely studied for a long time and the literature concerning this subject is rich. Here we mention only a few papers in the topic (in the chronological order): [4, [20, 44, [4], 6], $\S 3$ and $\S 4$ of Chapter 5 in [15], 46].

It is assumed that the reader is familiar with the basics on von Neumann algebras as well as operators affiliated with them. In particular, the reader should know that, according to a result due to Murray and von Neumann [36, all operators affiliated with a fixed finite type I von Neumann algebra form a *-algebra (with natural algebraic operations). For another proof, see e.g. 34. Since our work strongly depends on reduction theory of von Neumann algebras due to von Neumann himself [53, we expect that the reader knows a part of this theory that concerns finite type I algebras.

The paper is organized as follows. Section 2 discusses, in more detail and more generally, compatible functions. We prove there a result (Theorem 2.1) which the uniqueness part of Theorem 1.1 depends on. The third part is devoted to the proof of a generalization (and a strenthening; see Theorem 3.1) of Theorem 1.1 (and of Theorem 1.1 itself). Section 4 discusses a new concept of operator-valued spectra of operators. We define there the spectral measure of a finite tuple $\mathrm{T}$ of operators affiliated with a finite type I von Neumann algebra, formulate a spectral theorem for $\mathrm{T}$ (Theorem 4.11) and define the principal spectrum of $\mathrm{T}$ as well as give its characterization (Proposition 4.13), which resembles one of classical properties of the (usual) spectrum of a normal operator. It is also shown how such a tuple makes (in an ambiguous way) the underlying Hilbert space a left module over the $C^{*}$-product of all $\mathscr{M}_{n}$.

Notation and terminology. In this paper all Hilbert spaces are complex. An operator means a closed densely defined (unless otherwise stated) linear operator acting in a Hilbert space. An operator $T$ in a Hilbert space $\mathcal{H}$ is affiliated with a von Neumann algebra $\mathcal{M}$ of operators on $\mathcal{H}$ if $U T U^{-1}=T$ for any unitary operator $U$ from the commutant $\mathcal{M}^{\prime}$ of $\mathcal{M}$. The algebra of all operators affiliated with a finite 
type I von Neumann algebra $\mathcal{M}$ shall be denoted by $\hat{\mathcal{M}}$, and "+" and "." will stand for, respectively, the addition and the multiplication of $\hat{\mathcal{M}}$. When $\mathrm{T}=\left(T_{s}\right)_{s \in S}$ is a system of bounded operators on a Hilbert space $\mathcal{H}$, we use $\mathcal{W}(T)$ to denote the smallest von Neumann algebra on $\mathcal{H}$ that contains each of the operators $T_{s}$. If $T_{s}$ are merely closed and densely defined in $\mathcal{H}$ (and still $\mathrm{T}=\left(T_{s}\right)_{s \in S}$ ), by $\mathcal{W}^{\prime}(\mathrm{T})$ we denote the set of all bounded operators $X$ on $\mathcal{H}$ for which $X T_{s} \subset T_{s} X$ and $X^{*} T_{s} \subset T_{s} X^{*}$ for all $s \in S$. For any $\operatorname{ring} \mathscr{R}, \mathcal{Z}(\mathscr{R})$ stands for the center of $\mathscr{R}$. We use $\mathcal{W}^{\prime \prime}(\mathrm{T})$ to denote the commutant $\left(\mathcal{W}^{\prime}(\mathrm{T})\right)^{\prime}$ of $\mathcal{W}^{\prime}(\mathrm{T})$. Recall that both $\mathcal{W}^{\prime}(\mathrm{T})$ and $\mathcal{W}^{\prime \prime}(\mathrm{T})$ are von Neumann algebras on $\mathcal{H}$, and that $\mathcal{W}^{\prime \prime}(\mathrm{T})=\mathcal{W}(\mathrm{T})$ (by von Neumann's double commutant theorem) provided T consists of bounded operators. Whenever $x$ is a normal element in a unital $C^{*}$-algebra and $u: D \rightarrow \mathbb{C}$ (where $D$ is a set in $\mathbb{C}$ ) is a continuous function whose domain $D$ contains the spectrum $\sigma(x)$ of $x$, by $u[x]$ we denote the effect of acting of $u$ on $x$ within the classical functional calculus. For any topological space $X, \mathfrak{B}(X)$ is reserved to denote the $\sigma$-algebra of all Borel sets in $X$, that is, $\mathfrak{B}(X)$ is the smallest $\sigma$-algebra of subsets of $X$ that contains all open sets. A function $f: X \rightarrow Y$ between topological spaces $X$ and $Y$ is Borel if $f^{-1}(B) \in \mathfrak{B}(X)$ for any $B \in \mathfrak{B}(Y)$.

All notations and terminologies introduced earlier in this section are obligatory. Additionally, we denote by $I_{n}$ the unit $n \times n$ matrix.

\section{Compatible matrix FUnCtions}

First we shall extend the concept of compatible functions introduced in the previous part. To this end, we reserve $\ell$ and $\ell^{\prime}$ to denote lengths of tuples of matrices (as well as of operators). So, $\ell$ and $\ell^{\prime}$ are arbitrary positive integers. Let $\mathscr{M}^{(\ell)}$ stand for the topological disjoint union $\bigsqcup_{n=1}^{\infty} \mathscr{M}_{n}^{\ell}$ of the product spaces $\mathscr{M}_{n}^{\ell}$. $\mathscr{M}^{(\ell)}$ is a Polish (that is, separable completely metrizable) space. Recall that $\mathscr{M}^{(1)}=\mathscr{M}$. The space $\mathscr{M}^{(\ell)}$ shall be equipped with ingredients $d$ (the degree map), " $\oplus$ " (the addition) and "." (the unitary action) defined below (according to the terminology of [42], $\left(\mathscr{M}^{(\ell)}, d, \oplus,.\right)$ is a tower $)$. For any $\mathrm{A}=\left(A_{k}\right)_{k=1}^{\ell} \in \mathscr{M}^{(\ell)}$ we denote by $d(\mathrm{~A})$ a common degree of the matrices $A_{k}$ (so, $d(\mathrm{~A}) \stackrel{\text { def }}{=} n$ for $\mathrm{A} \in \mathscr{M}_{n}^{\ell}$ ). Further, for each $U \in \mathscr{U}_{d(\mathrm{~A})}$, the tuple $U$.A is defined as $\left(U A_{k} U^{-1}\right)_{k=1}^{\ell} \in \mathscr{M}^{(\ell)}$ (observe that $d(U . \mathrm{A})=d(\mathrm{~A}))$. Finally, if, in addition, also $\mathrm{B}=\left(B_{k}\right)_{k=1}^{\ell}$ is a member of $\mathscr{M}^{(\ell)}$, the tuple $\mathrm{A} \oplus \mathrm{B}$ is defined coordinatewise, that is, $\mathrm{A} \oplus \mathrm{B} \stackrel{\text { def }}{=}\left(A_{k} \oplus B_{k}\right)_{k=1}^{\ell}$. Additionally, $\|\mathrm{A}\|$ will stand for the maximum of all $\left\|A_{k}\right\|$.

When $\mathscr{S}$ is an arbitrary subset of $\mathscr{M}^{(\ell)}$, a function $f: \mathscr{S} \rightarrow \mathscr{M}^{\left(\ell^{\prime}\right)}$ is said to be compatible if

- $d(f(\mathbf{X}))=d(\mathbf{X})$ for each $\mathbf{X} \in \mathscr{S}$

- $f\left(U \cdot\left(\bigoplus_{j=1}^{s} \mathrm{X}_{j}\right)\right)=U \cdot\left(\bigoplus_{j=1}^{s} f\left(\mathrm{X}_{j}\right)\right)$ for all finite systems $\mathrm{X}_{1}, \ldots, \mathrm{X}_{s} \in \mathscr{S}$ and $U \in \mathscr{U}_{N}$ with $N=\sum_{j=1}^{s} d\left(\mathrm{X}_{j}\right)$ such that $\bigoplus_{j=1}^{s} \mathrm{X}_{j} \in \mathscr{S}$.

(Compatible functions, in a more general context, were introduced in 42 to show that certain algebras of such functions serve as models for all subhomogeneous $C^{*}$-algebras.) The function $f$ is bounded if $\left(\|f\| \stackrel{\text { def }}{=}\right.$ ) $\sup _{\mathbf{X} \in \mathscr{S}}\|f(\mathbf{X})\|<\infty$ (where $\sup (\varnothing) \stackrel{\text { def }}{=} 0)$, and $f$ is locally bounded if, for any positive real constant $R$, the restriction of $f$ to $\mathscr{S} \cap \mathscr{B}^{(\ell)}(R)$ is bounded where $\mathscr{B}^{(\ell)}(R) \stackrel{\text { def }}{=}\left\{\mathrm{X} \in \mathscr{M}^{(\ell)}:\|\mathrm{X}\| \leqslant\right.$ $R\}$. Finally, let $\mathscr{F}_{\ell, \ell^{\prime}}$ (resp. $\mathscr{F}_{\ell, \ell^{\prime}}^{l o c} ; \mathscr{F}_{\ell, \ell^{\prime}}^{b d}$ ) denote the family of all (resp. all locally bounded; all bounded) compatible Borel functions $f: \mathscr{M}^{(\ell)} \rightarrow \mathscr{M}^{\left(\ell^{\prime}\right)}$. It is easy to see that $\mathscr{F}_{\ell, \ell^{\prime}}$ is a unital $*$-algebra when the algebraic operations are defined as follows: if $f, g \in \mathscr{F}_{\ell, \ell^{\prime}}, \alpha \in \mathbb{C}, \mathbf{X} \in \mathscr{M}^{(\ell)}$ and $f(\mathrm{X})=\left(A_{1}, \ldots, A_{\ell^{\prime}}\right)$ and $g(\mathrm{X})=$ 
$\left(B_{1}, \ldots, B_{\ell^{\prime}}\right)$, then

$$
\begin{aligned}
& (f+g)(\mathrm{X}) \stackrel{\text { def }}{=}\left(A_{1}+B_{1}, \ldots, A_{\ell^{\prime}}+B_{\ell^{\prime}}\right), \\
& (\alpha \cdot f)(\mathrm{X}) \stackrel{\text { def }}{=}\left(\alpha A_{1}, \ldots, \alpha A_{\ell^{\prime}}\right), \\
& (f \cdot g)(\mathrm{X}) \stackrel{\text { def }}{=}\left(A_{1} B_{1}, \ldots, A_{\ell^{\prime}} B_{\ell^{\prime}}\right), \\
& \left(f^{*}\right)(\mathrm{X}) \stackrel{\text { def }}{=}\left(A_{1}^{*}, \ldots, A_{\ell^{\prime}}^{*}\right)
\end{aligned}
$$

(the unit of $\mathscr{F}_{\ell, \ell^{\prime}}$ is a function that is constantly equal to $\left(I_{n}, \ldots, I_{n}\right) \in \mathscr{M}_{n}^{\ell^{\prime}}$ on $\left.\mathscr{M}_{n}^{\ell}\right)$. Moreover, $\mathscr{F}_{\ell, \ell^{\prime}}$ is sequentially closed in the pointwise convergence topology of $\left(\mathscr{M}^{\left(\ell^{\prime}\right)}\right)^{\mathscr{M}^{(\ell)}}$; that is, if $f_{1}, f_{2}, \ldots \in \mathscr{F}_{\ell, \ell^{\prime}}$ converge pointwise to $f: \mathscr{M}^{(\ell)} \rightarrow \mathscr{M}^{\left(\ell^{\prime}\right)}$, then $f \in \mathscr{F}_{\ell, \ell^{\prime}}$ as well. This implies that $\mathscr{F}_{\ell, \ell^{\prime}}^{b d}$ is a unital $C^{*}$-algebra (with the norm $\|\cdot\|$ defined above). For simplicity, we put $\mathscr{F} \stackrel{\text { def }}{=} \bigcup_{\ell} \bigsqcup_{\ell^{\prime}} \mathscr{F} \ell, \ell^{\prime}$ where $\ell$ and $\ell^{\prime}$ run over all positive integers. Whenever $f_{1}, \ldots, f_{\ell^{\prime}}$ is a finite system of functions in $\mathscr{F}_{\ell, 1}$, by $\left(f_{k}\right)_{k=1}^{\ell^{\prime}}$ we shall denote their diagonal function; that is, $\left(f_{k}\right)_{k=1}^{\ell^{\prime}}: \mathscr{M}^{(\ell)} \rightarrow \mathscr{M}^{\left(\ell^{\prime}\right)}$ and $\left(f_{k}\right)_{k=1}^{\ell^{\prime}}(\mathbf{X}) \stackrel{\text { def }}{=}\left(f_{1}(\mathbf{X}), \ldots, f_{\ell^{\prime}}(\mathbf{X})\right)$ for $\mathbf{X} \in \mathscr{M}^{(\ell)}$. Finally, for any positive integer $j \leqslant \ell, \pi_{j}^{(\ell)}: \mathscr{M}^{(\ell)} \rightarrow \mathscr{M}$ will stand for the projection onto the $j$ th coordinate; that is, $\pi_{j}^{(\ell)}\left(X_{1}, \ldots, X_{\ell}\right) \stackrel{\text { def }}{=} X_{j}$.

Below we collect three additional properties of the class $\mathscr{F}$, which are relevant in our further investigations. Their proofs are straightforward and thus we skip them.

- If $f_{1}, \ldots, f_{\ell^{\prime}}$ is a finite collection of functions that belong to $\mathscr{F}_{\ell, 1}$, then $\left(f_{k}\right)_{k=1}^{\ell^{\prime}} \in \mathscr{F}_{\ell, \ell^{\prime}}$.

- If $f$ and $g$ belong to, respecrtively, $\mathscr{F}_{\ell, \ell^{\prime}}$ and $\mathscr{F}_{\ell^{\prime}, \ell^{\prime \prime}}$, then $g \circ f$ is well defined and belongs to $\mathscr{F}_{\ell, \ell^{\prime \prime}}$.

- If $f \in \mathscr{F}_{\ell, 1}$ is such that $f(\mathrm{X})$ is an invertible matrix for any $\mathrm{X} \in \mathscr{M}^{(\ell)}$, then the function $(f)^{-1}: \mathscr{M}^{(\ell)} \rightarrow \mathscr{M}$ defined by $(f)^{-1}(\mathrm{X}) \stackrel{\text { def }}{=}(f(\mathrm{X}))^{-1}$ belongs to $\mathscr{F}_{\ell, 1}$ as well.

The main result of this section is the following

2.1. Theorem. Let $\mathscr{F}_{0}$ denote one of $\mathscr{F}_{\ell, 1}$ or $\mathscr{F}_{\ell, 1}^{l o c}$. Assume $\mathscr{E}$ is a unital *subalgebra of $\mathscr{F}_{0}$ which satisfies each of the following conditions:

(E0) $\pi_{j}^{(\ell)} \in \mathscr{E}$ for each positive integer $j \leqslant \ell$;

(E1) if all values of $f \in \mathscr{E}$ are invertible matrices and $(f)^{-1} \in \mathscr{F}_{0}$, then $(f)^{-1}$ belongs to $\mathscr{E}$;

(E2) whenever $f_{n} \in \mathscr{E}$ are uniformly bounded and converge pointwise to a function $f \in \mathscr{F}_{0}$, then $f \in \mathscr{E}$.

Then $\mathscr{E}=\mathscr{F}_{0}$.

Although the above result is intuitive, its proof is a little bit complicated and based on a certain selector theorem which shall be formulated after introducing necessary notions.

A tuple $\mathrm{X} \in \mathscr{M}^{(\ell)}$ is reducible if there exist $\mathrm{A}, \mathrm{B} \in \mathscr{M}^{(\ell)}$ and a unitary matrix $U \in \mathscr{U}_{d(\mathrm{X})}$ for which $\mathrm{X}=U .(\mathrm{A} \oplus \mathrm{B})$; otherwise $\mathrm{X}$ is irreducible (see 42]; the latter is similar to the notion of an irreducible representation of a $C^{*}$-algebra). Two tuples $\mathrm{A}$ and $\mathrm{B}$ in $\mathscr{M}^{(\ell)}$ are said to be unitarily equivalent, in symbols $\mathrm{A} \equiv \mathrm{B}$, if $\mathrm{B}=V$. $\mathrm{A}$ for some $V \in \mathscr{U}_{d(\mathrm{~A})}$ (in particular, $d(\mathrm{~A})=d(\mathrm{~B})$ provided $\left.\mathrm{A} \equiv \mathrm{B}\right)$. A set $\mathscr{K} \subset \mathscr{M}^{(\ell)}$ is called by us a kernel of $\mathscr{M}^{(\ell)}$ if $\mathscr{K}$ is a selector for irreducible tuples (with respect to the unitary action); that is, $\mathscr{K}$ is a kernel if it consists of irreducible tuples and for any irreducible tuple $\mathrm{X} \in \mathscr{M}^{(\ell)}$ there is a unique tuple $\mathrm{Y} \in \mathscr{K}$ such that $\mathrm{X} \equiv \mathrm{Y}$. 
What we need is the next result. We recall that a subset of a topological space is $\sigma$-compact if it is a countable union of compact sets.

2.2. Proposition. For any $\ell, \mathscr{M}^{(\ell)}$ contains a $\sigma$-compact kernel.

The proof presented below is in the spirit of the argument from [47. The existence of kernels that are $\mathscr{G}_{\delta}$-sets in $\mathscr{M}^{(\ell)}$ may readily be deduced from Corollary 1 in $\S 2$ of Chapter XIV in [30] or from [7].

Proof. Denote by $\mathcal{Q}$ the collection of all polynomials in $2 \ell$ noncommuting variables whose all coefficients belong to $\mathbb{Q}+\mathrm{i} \mathbb{Q}$, and by $\mathscr{I}^{(\ell)}$ the set of all tuples $\mathrm{X} \in \mathscr{M}^{(\ell)}$ that are irreducible. It is easy to show that $\mathscr{I}^{(\ell)}$ is an open set in $\mathscr{M}^{(\ell)}$ and therefore

$$
\mathscr{I}^{(\ell)} \text { is } \sigma \text {-compact. }
$$

Note that a tuple $\left(X_{1}, \ldots, X_{\ell}\right) \in \mathscr{M}_{n}^{\ell}\left(\subset \mathscr{M}^{(\ell)}\right)$ is irreducible iff the set

$$
\left\{p\left(X_{1}, \ldots, X_{\ell}, X_{1}^{*}, \ldots, X_{\ell}^{*}\right): p \in \mathcal{Q}\right\}
$$

is dense in $\mathscr{M}_{n}$. Further, let $\mathscr{V}_{0}$ be the set of all matrices $X=\left[x_{j k}\right] \in \mathscr{M}$ such that: (ax1) $x_{j k}+\bar{x}_{k j}=0$ for all distinct $j$ and $k$;

(ax2) $\operatorname{Re}\left(x_{j j}\right)>\operatorname{Re}\left(x_{k k}\right)$ whenever $j>k$;

(ax3) $x_{1 k}$ is a positive real number for $k>1$.

Let us note here the following property of $\mathscr{V}_{0}$ :

(P0) if $X \in \mathscr{V}_{0}, Y=\left[y_{j k}\right] \in \mathscr{M}$ and $U \in \mathscr{U}_{d(X)}$ are such that

$(\operatorname{ax} 0 ') Y=U . X ;$ and

$\left(\mathrm{ax} 1^{\prime}\right) y_{j k}+\bar{y}_{k j}=0$ for all distinct $j$ and $k$; and

$\left(\operatorname{ax} 2{ }^{\prime}\right) \operatorname{Re}\left(y_{j j}\right) \geqslant \operatorname{Re}\left(y_{k k}\right)$ whenever $j \geqslant k$; and

(ax3') $y_{1 k}$ is a nonnegative real number for $k>1$,

then $Y=X$ and $U$ is a scalar multiple of the unit matrix.

To show $(\mathrm{P} 0)$, assume $X=\left[x_{j k}\right]$. Since $Y=U . X$, we conclude that $(n \stackrel{\text { def }}{=}) d(X)=$ $d(Y)$ and $Y+Y^{*}=U \cdot\left(X+X^{*}\right)$. Consequently, the spectra of $X+X^{*}$ and $Y+Y^{*}$ (that is, the sets of all their eigenvalues) coincide. But (ax1) and (ax1') imply that both $X+X^{*}$ and $Y+Y^{*}$ are diagonal matrices. So, (ax2) and (ax2') yield that $\operatorname{Re}\left(x_{j j}\right)=\operatorname{Re}\left(y_{j j}\right)$ for each $j \in\{1, \ldots, n\}$, which means that $X+X^{*}=Y+Y^{*}$. Hence $U$ commutes with a diagonal matrix all of whose diagonal entries are different. We infer that $U$ is a diagonal matrix as well. So, if $n>1$ and $\lambda_{1}, \ldots, \lambda_{n}$ are the consecutive entries of the diagonal of $U$, then $y_{1 k}=\lambda_{1} x_{1 k} \bar{\lambda}_{k}$ for each $k>1$. Since both the numbers $y_{1 k}$ and $x_{1 k}$ are real and nonnegative and $x_{1 k} \neq 0$ (see (ax3) and (ax3')), we see that $\lambda_{k}=\lambda_{1}$, which means that $U$ is a scalar multiple of the identity matrix and, consequently, $Y=X$. The proof of $(\mathrm{P} 0)$ is complete.

Now put $\mathscr{V} \stackrel{\text { def }}{=}\left\{U . X: X \in \mathscr{V}_{0}, U \in \mathscr{U}_{d(X)}\right\}$. We claim that $\mathscr{V}$ is open in $\mathscr{M}$. To convince oneself of that, it suffices to check that $\mathscr{V} \cap \mathscr{M}_{N}$ is open in $\mathscr{M}_{N}$ for each $N>0$. Suppose, on the contrary, that $\mathscr{V} \cap \mathscr{M}_{N}$ is not open. This means that there is $X \in \mathscr{V} \cap \mathscr{M}_{N}$ and a sequence of matrices $X_{n} \in \mathscr{M}_{N} \backslash \mathscr{V}$ that converge to $X$. Since any selfadjoint matrix is unitarily equivalent to diagonal, we see that there is $U_{n} \in \mathscr{U}_{N}$ such that $U_{n} .\left(X_{n}+X_{n}^{*}\right)$ is a diagonal matrix whose consecutive diagonal entries are monotone increasing (that is, nondecreasing). Further, there is a diagonal matrix $V_{n} \in \mathscr{U}_{N}$ for which all entries, apart from the first, of the first row of $\left(V_{n} U_{n}\right) \cdot X_{n}$ are real and nonnegative. Observe that then $\left(V_{n} U_{n}\right) \cdot\left(X_{n}+\right.$ $\left.X_{n}^{*}\right)=U_{n} \cdot\left(X_{n}+X_{n}^{*}\right)$ (because $V_{n}$ and $U_{n} \cdot\left(X_{n}+X_{n}^{*}\right)$ are diagonal). Passing to a subsequence, we may and do assume that $W_{n} \stackrel{\text { def }}{=} V_{n} U_{n}$ converge to $W \in \mathscr{U}_{N}$. Then the matrix $Y \stackrel{\text { def }}{=} W . X$ coincides with $\lim _{n \rightarrow \infty} W_{n} \cdot X_{n}$ and therefore has properties (ax1')-(ax3'). But $X \in \mathscr{V}$ which means that $X^{\prime} \stackrel{\text { def }}{=} U . X$ belongs to $\mathscr{V}_{0}$ for some 
$U \in \mathscr{U}_{N}$. So, $Y=\left(W U^{-1}\right) \cdot X^{\prime}$ and $(\mathrm{P} 0)$ shows that $Y=X^{\prime}$. This means that $Y \in \mathscr{V}_{0}$, from which we infer that all but a finite number of the matrices $W_{n} . X_{n}$ also belong to $\mathscr{V}_{0}$, which contradicts the assumption that $X_{n} \notin \mathscr{V}$. So, $\mathscr{V}$ is indeed open.

Of course, $\mathscr{V}$ meets each of $\mathscr{M}_{n}$. Now arrange all members of $\mathcal{Q}$ in a sequence $p_{1}, p_{2}, \ldots$ and for any positive integer $n$ denote by $\mathscr{I}_{n}$ the set of all $\mathbf{X}=$ $\left(X_{1}, \ldots, X_{\ell}\right) \in \mathscr{I}^{(\ell)}$ such that $p_{n}\left(X_{1}, \ldots, X_{\ell}, X_{1}^{*}, \ldots, X_{\ell}^{*}\right) \in \mathscr{V}$. Since $\mathscr{V}$ is open, we see that each of $\mathscr{I}_{n}$ is open in $\mathscr{I}^{(\ell)}$. Moreover, we conclude from the previous remarks that the sets $\mathscr{I}_{n}$ cover $\mathscr{I}^{(\ell)}$. We put $\mathscr{D}_{n} \stackrel{\text { def }}{=} \mathscr{I}_{n} \backslash \mathscr{I}_{n-1}$ where $\mathscr{I}_{0} \stackrel{\text { def }}{=} \varnothing$. Observe that:

(D1) the sets $\mathscr{D}_{n}$ are pairwise disjoint and cover $\mathscr{I}^{(\ell)}$; and

(D2) each of $\mathscr{D}_{n}$ is $\sigma$-compact (by (2-1)); and

(D3) if $\mathrm{X} \in \mathscr{D}_{n}$ and $U \in \mathscr{U}_{d(\mathrm{X})}$, then $U . \mathrm{X} \in \mathscr{D}_{n}$ as well.

Let $\mathrm{X} \in \mathscr{D}_{n}$. Since $p_{n}(\mathrm{X}) \in \mathscr{V}$, there is $U_{\mathrm{X}} \in \mathscr{U}_{d(\mathrm{X})}$ for which $U_{\mathrm{X}} \cdot p_{n}(\mathrm{X}) \in \mathscr{V}_{0}$ (moreover, $U_{\mathbf{X}}$ is unique up to a scalar multiple, thanks to $(\mathrm{P} 0)$ ). We now put

$$
\mathscr{K}^{\text {def }}=\left\{U_{X} \cdot X: X \in \mathscr{I}^{(\ell)}\right\} .
$$

We see that $\mathscr{K}$ consists of irreducible tuples and for any $\mathrm{X} \in \mathscr{I}^{(\ell)}, \mathscr{K}$ contains a tuple unitarily equivalent to $\mathbf{X}$ (by (D1)). Further, notice that if $U_{\mathbf{X}} \cdot \mathbf{X}$ belongs to $\mathscr{D}_{n}$, then $\mathrm{X} \in \mathscr{D}_{n}$ as well (by (D3)) and hence $p_{n}\left(U_{\mathrm{X}} \cdot \mathrm{X}\right)=U_{\mathbf{X}} \cdot p_{n}(\mathrm{X}) \in \mathscr{V}_{0}$. This shows that $p_{n}(\mathrm{~A}) \in \mathscr{V}_{0}$ for any $\mathrm{A} \in \mathscr{K} \cap \mathscr{D}_{n}$. Now assume $\mathrm{A}$ and $\mathrm{B}$ are two tuples in $\mathscr{K}$ that are unitarily equivalent. Then, by (D1) and (D3), there is a unique $n$ such that $\mathrm{A}, \mathrm{B} \in \mathscr{D}_{n}$. Take $V \in \mathscr{U}_{d(\mathrm{~A})}$ for which $\mathrm{B}=V$.A. Then also $p_{n}(\mathrm{~B})=V \cdot p_{n}(\mathrm{~A})$. But, as shown above, both $p_{n}(\mathrm{~A})$ and $p_{n}(\mathrm{~B})$ belong to $\mathscr{V}_{0}$. Thus, we infer from $(\mathrm{P} 0)$ that $V$ is a scalar multiple of the unit matrix, and hence $\mathrm{Y}=\mathrm{X}$. This shows that $\mathscr{K}$ is a kernel. It remains to check that $\mathscr{K} \cap \mathscr{D}_{m} \cap \mathscr{M}_{n}^{\ell}$ is $\sigma$-compact for each $m$ and $n$ (because then $\mathscr{K}$ itself be $\sigma$-compact). To this end, we consider the map

$$
\Phi: \mathscr{U}_{n} \times \mathscr{M}_{n}^{\ell} \ni\left(U ; X_{1}, \ldots, X_{\ell}\right) \mapsto U \cdot p_{m}\left(X_{1}, \ldots, X_{\ell}, X_{1}^{*}, \ldots, X_{\ell}^{*}\right) \in \mathscr{M}_{n} .
$$

Notice that $\Phi$ is proper (i.e., the inverse image of a compact set in $\mathscr{M}_{n}$ under $\Phi$ is compact). It is an easy observation that $\mathscr{V}_{0}$ is $\sigma$-compact (it is even locally compact) and therefore $\mathscr{L} \stackrel{\text { def }}{=} \Phi^{-1}\left(\mathscr{V}_{0}\right)$ is also $\sigma$-compact. Consequently, the set $\{U . \mathrm{X}:(U, \mathrm{X}) \in \mathscr{L}\}$ is $\sigma$-compact as well. But, the last aforementioned set coincides with $\mathscr{K}$ (because for any $\mathrm{X} \in \mathscr{D}_{m}$ the unitary matrix $U_{\mathrm{X}}$ is unique up to a scalar multiple) and we are done.

We shall also need the following lemma, which is a special case of a variation of the Stone-Weierstrass theorem for $C^{*}$-algebras proved in 38 .

2.3. Lemma. Let $X$ be a compact Hausdorff space and let $\mathcal{A}$ be a unital $C^{*}$-algebra. Let $\mathcal{E}$ be a $*$-subalgebra of $C(X, \mathcal{A})$ such that for any two points $x$ and $y$ of $X$ there is $f \in \mathcal{E}$ with $f(x)=1$ and $f(y)=0$. Then the closure of $\mathcal{E}$ in $C(X, \mathcal{A})$ coincides with the *-algebra $\Delta_{2}(\mathcal{E})$ of all maps $u \in C(X, \mathcal{A})$ such that for any $x, y \in X$ and each $\varepsilon>0$ there exists $v \in \mathcal{E}$ with $\|v(x)-u(x)\|<\varepsilon$ and $\|v(y)-u(y)\|<\varepsilon$.

The reader interested in other results in a similar spirit as above is referred to Theorem 1.4 in [16] or Corollary 11.5.3 in [11]. For other variations of the StoneWeierstrass thorem settled in $C^{*}$-algebras, consult, e.g., [17], $§ 4.7$ in [48, [35] or 45].

2.4. Proposition. Let $\mathscr{K}$ be a $\sigma$-compact kernel of $\mathscr{M}^{(\ell)}$ and let $\mathscr{F}_{0}$ denote either the *-algebra of all Borel functions $u: \mathscr{K} \rightarrow \mathscr{M}$ such that

$$
d(u(\mathbf{X}))=d(\mathbf{X}) \quad(\mathbf{X} \in \mathscr{K})
$$


or the *-algebra of all locally bounded such functions. If $\mathcal{E}$ is a unital *-subalgebra of $\mathscr{F}_{0}$ such that $\left.\pi_{k}^{(\ell)}\right|_{\mathscr{K}} \in \mathcal{E}$ for any $k \leqslant \ell$ and conditions (E1)-(E2) hold, then $\mathcal{E}=\mathscr{F}_{0}$.

Proof. Below we shall involve the concept of $\mathfrak{b}$-transform, introduced by us in [37]. Namely, for any matrix $X \in \mathscr{M}_{n}$, let $\mathfrak{b}(X) \stackrel{\text { def }}{=} X\left(I_{n}+|X|\right)^{-1}$ (where $\left.|X| \stackrel{\text { def }}{=} \sqrt{X^{*} X}\right)$. The $\mathfrak{b}$-transform for tuples of matrices (or operators) is defined coordinatewise, that is, $\mathfrak{b}\left(X_{1}, \ldots, X_{\ell}\right) \stackrel{\text { def }}{=}\left(\mathfrak{b}\left(X_{1}\right), \ldots, \mathfrak{b}\left(X_{\ell}\right)\right)$. What is relevant for us is that:

$(\mathfrak{b} 1)$ for any $\mathrm{X} \in \mathscr{M}^{(\ell)},\|\mathfrak{b}(\mathrm{X})\| \leqslant 1$;

$(\mathfrak{b} 2)$ if $\mathrm{X} \in \mathscr{M}^{(\ell)}$ is irreducible, then so is $\mathfrak{b}(\mathrm{X})$;

(b3) two tuples $\mathrm{X}$ and $\mathrm{Y}$ in $\mathscr{M}^{(\ell)}$ are unitarily equivalent iff so are $\mathfrak{b}(\mathrm{X})$ and $\mathfrak{b}(\mathrm{Y})$.

We shall use these properties below, where $\mathfrak{b}$ shall be considered as a function of $\mathscr{K}$ into $\mathscr{M}^{(\ell)}$. We shall also use $j$ to denote the unit of $\mathcal{E}\left(j(\mathrm{X})=I_{d(\mathrm{X})}\right.$ for any $\mathrm{X} \in \mathscr{K})$. More generally, for any Borel set $\mathscr{A}$ in $\mathscr{K}$, we denote by $j_{\mathscr{A}}$ the function of $\mathscr{K}$ into $\mathscr{M}$ that coincides with $j$ on $\mathscr{A}$ and vanishes at each point of $\mathscr{K} \backslash \mathscr{A}$.

We fix a compact subset $\mathscr{L}$ of $\mathscr{K}$. Our aim is to show that

(L0) $j_{\mathscr{L}} u \in \mathcal{E}$ for any bounded Borel function $u: \mathscr{K} \rightarrow \mathscr{M}$ that satisfies (2-2).

To this end, first observe that

( $\star$ ) for any two distinct element $\mathrm{X}$ and $\mathrm{Y}$ in $\mathscr{K}$, there is a polynomial $p$ in $2 \ell$ noncommuting variables with $p\left(\mathfrak{b}(\mathbf{X}), \mathfrak{b}(\mathbf{X})^{*}\right)=0$ and $p\left(\mathfrak{b}(\mathbf{Y}), \mathfrak{b}(\mathbf{Y})^{*}\right)=I_{d(\mathrm{Y})}$ (because $\mathfrak{b}(X)$ and $\mathfrak{b}(Y)$ are irreducible and unitarily inequivalent, see $(\mathfrak{b} 2)$ and $(\mathfrak{b} 3)$; cf. Proposition 4.2.5 in [11]). Since $\left.\pi_{k}^{(\ell)}\right|_{\mathscr{K}}$ belongs to $\mathcal{E}$ for any $k,(\mathrm{E} 1)-(\mathrm{E} 2)$ imply that

$$
\pi_{k}^{(\ell)} \circ \mathfrak{b} \in \mathcal{E}
$$

as well. Indeed, $j+\left(\pi_{k}^{(\ell)}\right)^{*} \pi_{k}^{(\ell)}$ belongs to $\mathcal{E}$ and has all its values invertible. Thus, it follows from (E1) that the function

$$
v_{k}: \mathscr{K} \ni\left(X_{1}, \ldots, X_{\ell}\right) \mapsto\left(I_{d\left(X_{k}\right)}+X_{k}^{*} X_{k}\right)^{-1} \in \mathscr{M}
$$

is a member of $\mathcal{E}$. Observe that $v_{k}$ is a nonnegative (selfadjoint) element of the $C^{*}$ algebra $\mathcal{E}_{b d}$ of all bounded functions in $\mathcal{E}$ whose norms do not exceed 1 ( $\mathcal{E}_{b d}$ is indeed a $C^{*}$-algebra thanks to $\left.(\mathrm{E} 2)\right)$. So, $v_{k}^{\prime} \stackrel{\text { def }}{=} u\left[v_{k}\right]$ belongs to $\mathcal{E}_{b d}$ where $u:[0,1] \rightarrow$ $[0, \infty)$ is given by $u(t)=\frac{\sqrt{t}}{\sqrt{t}+\sqrt{1-t}}$. Direct calculations show that $v_{k}^{\prime}\left(X_{1}, \ldots, X_{\ell}\right)=$ $\left(I_{d\left(X_{k}\right)}+\left|X_{k}\right|\right)^{-1}$. Consequently, $\left.\pi_{k}^{(\ell)}\right|_{\mathscr{K}} v_{k}^{\prime} \in \mathcal{E}$, which is equivalent to (2-3) . Having this, we conclude that the function $\tilde{p}: \mathscr{K} \ni \mathbf{X} \mapsto p\left(\mathfrak{b}(\mathbf{X}), \mathfrak{b}(\mathbf{X})^{*}\right) \in \mathscr{M}$ belongs to $\mathcal{E}_{b d}$ when $p$ is any polynomial in $2 \ell$ noncommuting variables. Replacing $\tilde{p}$ by $\frac{1}{2}\left(\tilde{p}+\tilde{p}^{*}\right)$, we see that for any $\mathrm{X} \in \mathscr{L}$ and $\mathrm{Y} \in \mathscr{K} \backslash \mathscr{L}$ there is a selfadjoint continuous function $f_{\mathrm{X}, \mathrm{Y}} \in \mathcal{E}_{b d}$ such that $f_{\mathrm{X}, \mathrm{Y}}(\mathrm{X})=0$ and $f_{\mathrm{X}, \mathrm{Y}}(\mathrm{Y})=I_{d(\mathrm{Y})}$ (see $\left.(\star)\right)$. We may also assume that $0 \leqslant f_{X, Y} \leqslant j$, and $f_{X, Y}(Z)=0$ and $f_{X, Y}(\mathrm{~W})=I_{d(\mathrm{~W})}$ for $\mathrm{Z}$ and $W$ from, respectively, some neighbourhoods $U_{X, Y}$ of $X$ and $V_{X, Y}$ of $Y$ (because we may replace, if needed, $f_{\mathrm{X}, \mathrm{Y}}$ by $w\left[f_{\mathrm{X}, \mathrm{Y}}\right]$ where $\left.w(t)=\max (\min (3 t-1,1), 0)\right)$. Now it follows from the compactness of $\mathscr{L}$ that, when $\mathrm{Y}$ is fixed, there are a finite number of points $\mathrm{X}_{1}, \ldots, \mathrm{X}_{n} \in \mathscr{K}$ such that $\mathscr{L} \subset \bigcup_{k=1}^{n} U_{\mathrm{X}_{k}}$. We put $g_{\mathrm{Y}} \stackrel{\text { def }}{=}$ $j-\left(\prod_{k=1}^{n} f_{\mathrm{X}_{k}, \mathrm{Y}}\right)^{*}\left(\prod_{k=1}^{n} f_{\mathrm{X}_{k}, \mathrm{Y}}\right)$. Observe that $g_{\mathrm{Y}}$ is a selfadjoint member of $\mathcal{E}$, $0 \leqslant g_{\mathrm{Y}} \leqslant j, g_{\mathrm{Y}}(\mathrm{Z})=I_{d(\mathrm{Z})}$ for all $\mathrm{Z} \in \mathscr{K}$ and $g_{\mathrm{Y}}$ vanishes at each point of the neighbourhood $W_{\mathrm{Y}} \stackrel{\text { def }}{=} \bigcap_{k=1}^{n} V_{\mathrm{X}_{k}, \mathrm{Y}}$ of $\mathrm{Y}$. Further, we infer from the separability of $\mathscr{K}$ there there is a sequence $\mathrm{Y}_{1}, \mathrm{Y}_{2}, \ldots$ of elements of $\mathscr{K} \backslash \mathscr{L}$ for which $\bigcup_{k=1}^{\infty} W_{\mathrm{Y}_{k}}=$ $\mathscr{K} \backslash \mathscr{L}$. Notice that then the functions $h_{n} \stackrel{\text { def }}{=} \prod_{k=1}^{n} g_{\mathrm{Y}_{k}}$ belong to $\mathcal{E}$, are uniformly 
bounded and converge pointwise to $j_{\mathscr{L}}$. So, (E2) yields that

$$
j_{\mathscr{L}} \in \mathcal{E} .
$$

Now let $\mathcal{E}^{\prime}$ be the collection of all continuous functions from $\mathscr{L}$ into $\mathscr{M}$ which are restrictions of some functions from $\mathcal{E}_{b d}$. We want to show that $\mathcal{E}^{\prime}$ coincides with the $C^{*}$-algebra $\mathcal{D}$ of all continuous functions $u: \mathscr{L} \rightarrow \mathscr{M}$ for which (2-2) is fulfilled for any $\mathrm{X} \in \mathscr{L}$. To this end, take $N>0$ such that $\mathscr{L} \subset \bigcup_{n=1}^{N} \mathscr{M}_{n}$, put $R \stackrel{\text { def }}{=} N$ ! and $\mathcal{A} \stackrel{\text { def }}{=} \mathscr{M}_{R}$. We define a $*$-homomorphism $\Phi: \mathcal{D} \rightarrow C(\mathscr{L}, \mathcal{A})$ by

$$
(\Phi(f))(\mathbf{X}) \stackrel{\text { def }}{=} \underbrace{f(\mathbf{X}) \oplus \ldots \oplus f(\mathbf{X})}_{R / d(\mathbf{X})} .
$$

Since $\Phi$ is isometric, it is enough to check that $\Phi\left(\mathcal{E}^{\prime}\right)=\Phi(\mathcal{D})$. The previous reasoning (which starts from $(\star)$ ) shows that for any distinct elements $\mathrm{X}$ and $\mathrm{Y}$ of $\mathscr{L}$ there is a function $f \in \mathcal{E}_{b d}$ with $f(\mathrm{X})=I_{d(\mathrm{X})}$ and $f(\mathrm{Y})=0$. Then $\Phi\left(\left.f\right|_{\mathscr{L}}\right)(\mathrm{X})=I_{R}$ and $\Phi\left(\left.f\right|_{\mathscr{L}}\right)(\mathrm{Y})=0$. So, Lemma 2.3 implies that $\Phi\left(\mathcal{E}^{\prime}\right)$ coincides with $\Delta_{2}\left(\Phi\left(\mathcal{E}^{\prime}\right)\right) \subset$ $C(\mathscr{L}, \mathcal{A})\left(\mathcal{E}^{\prime}\right.$ is a $C^{*}$-algebra as the image of a $C^{*}$-subalgebra of $\mathcal{E}_{b d}$ under a $*$ homomorphism). But, since $\mathfrak{b}(\mathrm{X})$ is irreducible, for each matrix $T \in \mathscr{M}_{d(\mathrm{X})}$ we may find a polynomial $p$ in $2 \ell$ noncommuting variables such that $\tilde{p}(\mathrm{X})=T$. All these remarks show that

$$
\left\{(u(\mathrm{X}), u(\mathrm{Y})): u \in \Phi\left(\mathcal{E}^{\prime}\right)\right\}=\{(v(\mathrm{X}), v(\mathrm{Y})): v \in \Phi(\mathcal{D})\}
$$

and consequently $\Delta_{2}\left(\Phi\left(\mathcal{E}^{\prime}\right)\right)=\Phi(\mathcal{D})$. So, $\mathcal{E}^{\prime}=\mathcal{D}$. Combining this connection with (2-4), we obtain

$(\star \star)$ if $u \in \mathscr{F}_{0}$ vanishes at each point off $\mathscr{L}$ and its restriction to $\mathscr{L}$ is continuous, then $u \in \mathcal{E}$.

Further, denote by $\mathfrak{M}$ the family of all Borel sets $\mathscr{A}$ in $\mathscr{L}$ for which $j_{\mathscr{A}} \in \mathcal{E}$. Since $\mathcal{E}$ is an algebra that satisfies (E2) and contains $j_{\mathscr{L}}$, we readily get that $\mathfrak{M}$ is a $\sigma$-algebra of subsets of $\mathscr{L}$. So, to conclude that $\mathfrak{M}=\mathfrak{B}(\mathscr{L})$, it is enough to show that each closed subset of $\mathscr{L}$ belongs to $\mathfrak{M}$. But this simply follows from $(\star \star)$. Indeed, if $\mathscr{L}_{0}$ is a closed set in $\mathscr{L}$, there is a sequence of continuous functions $v_{n}: \mathscr{L} \rightarrow[0,1]$ that converge pointwise to the characteristic function of $\mathscr{L}_{0}$. Then each of the functions $q_{n}: \mathscr{K} \rightarrow \mathscr{M}$ given by $q_{n}(\mathrm{X})=v_{n}(\mathrm{X}) j(\mathrm{X})$ for $\mathrm{X} \in \mathscr{L}$ and $q_{n}(\mathrm{X})=0$ otherwise belongs to $\mathcal{E}$, thanks to $(\star \star)$, and hence $j_{\mathscr{L}_{0}} \in \mathcal{E}$, by (E2). So, $\mathfrak{M}=\mathfrak{B}(\mathscr{L})$ or, equivalently,

$$
j_{\mathscr{A}} \in \mathcal{E} \quad(\mathscr{A} \in \mathfrak{B}(\mathscr{L})) .
$$

Finally, let $u: \mathscr{K} \rightarrow \mathscr{M}$ be a bounded Borel function that satisfies (2-2). For fixed $\varepsilon>0$, we may find sequences $\mathscr{B}_{1}, \mathscr{B}_{2}, \ldots$ of pairwise disjoint Borel subsets of $\mathscr{L}$ that cover $\mathscr{L}$ and $T_{1}, T_{2}, \ldots$ of matrices such that for any $n$ and each $\mathrm{X} \in \mathscr{B}_{n}$, $d(\mathbf{X})=d\left(T_{n}\right)$ and $\left\|u(\mathbf{X})-T_{n}\right\| \leqslant \varepsilon$ (this implies that $\left.\sup _{n \geqslant 1}\left\|T_{n}\right\|<\infty\right)$. For each $n$, the function $\xi_{n}: \mathscr{K} \rightarrow \mathscr{M}$ which is constantly equal to $T_{n}$ on $\mathscr{B}_{n}$ and vanishes at each point off $\mathscr{B}_{n}$ belongs to $\mathcal{E}$, thanks to $(\star \star)$ and (2-5). We define $u_{\varepsilon}: \mathscr{K} \rightarrow \mathscr{M}$ as the pointwise limit of the series $\sum_{n=1}^{\infty} \xi_{n}$. Since the partial sums of the aforementioned series are uniformly bounded, we infer from (E2) that $u_{\varepsilon} \in \mathcal{E}$. Finally, it follows from our construction that $\left\|j_{\mathscr{L}} u-u_{\varepsilon}\right\| \leqslant \varepsilon$ and, consequently, $j_{\mathscr{L}} u \in \mathcal{E}$ (again by (E2)). This finishes the proof of (L0).

Having (L0), we can now easily finish the proof of the proposition. First take an arbitrary bounded Borel function $u: \mathscr{K} \rightarrow \mathscr{M}$ that satisfies (2-2). Since $\mathscr{K}$ is $\sigma$-compact, we may express $\mathscr{K}$ as the union of an ascending sequence of compact subsets $\mathscr{L}_{1}, \mathscr{L}_{2}, \ldots$ of $\mathscr{K}$. Then $j_{\mathscr{L}_{n}} u \in \mathcal{E}$, by (L0) and consequently $u$, as the pointwise limit of a uniformly bounded sequence of the functions $j_{\mathscr{L}_{n}} u$, belongs to $\mathcal{E}$ as well (again by (E2)). 
Finally, we take an arbitrary Borel function $u \in \mathscr{F}_{0}$. Let $v: \mathscr{K} \rightarrow \mathscr{M}$ and $w: \mathscr{K} \rightarrow \mathscr{M}$ be defined by $v(\mathbf{X}) \stackrel{\text { def }}{=} \mathfrak{b}(u(\mathbf{X}))$ and $w(\mathbf{X}) \stackrel{\text { def }}{=}\left(I_{d(\mathbf{X})}+|u(\mathbf{X})|\right)^{-1}$. The previous paragraph proves that $v, w \in \mathcal{E}$. Now (E1) implies that also $(w)^{-1}$ belongs to $\mathcal{E}$. Since $u=v \cdot(w)^{-1}$, we conclude that $u \in \mathcal{E}$ and we are done.

2.5. Corollary. Let $\mathscr{K}$ be a $\sigma$-compact kernel of $\mathscr{M}^{(\ell)}$. Every Borel function $u: \mathscr{K} \rightarrow \mathscr{M}$ that satisfies (2-2) admits a unique extension $\hat{u}$ to a function in $\mathscr{F}_{\ell, 1}$. Moreover, the assignment $u \mapsto \hat{u}$ establishes a one-to-one correspondence between Borel functions $u: \mathscr{K} \rightarrow \mathscr{M}$ satisfying (2-2) and functions from $\mathscr{F}_{\ell, 1}$. Moreover,

(ext1) $\hat{u} \in \mathscr{F}_{\ell, 1}^{\text {bd }}$ (resp. $\hat{u} \in \mathscr{F}_{\ell, 1}^{\text {loc }}$ ) iff $u$ is bounded (resp. $u$ is locally bounded), and $\|\hat{u}\|=\|u\|$;

(ext2) Borel functions $u_{n}: \mathscr{K} \rightarrow \mathscr{M}$ (for which (2-2) hold) converge pointwise to $u: \mathscr{K} \rightarrow \mathscr{M}$ if and only if the functions $\hat{u}_{n}$ converge so to $\hat{u}$.

Proof. First of all, note that for any $\mathrm{X} \in \mathscr{M}^{(\ell)}$ there are $U \in \mathscr{U}_{d(\mathrm{X})}$ and $\mathrm{X}_{1}, \ldots, \mathrm{X}_{p} \in$ $\mathscr{K}$ (for some $p>0$ ) such that

$$
\mathrm{X}=U \cdot\left(\mathrm{X}_{1} \oplus \ldots \oplus \mathrm{X}_{p}\right)
$$

This property implies that two functions from $\mathscr{F}_{\ell, 1}$ coincide provided their restrictions to $\mathscr{K}$ do so. Now let $\mathcal{E}$ consists of all Borel functions $u: \mathscr{K} \rightarrow \mathscr{M}$ that satisfy (2-2) and extend to some (necessarily unique) function $\hat{u} \in \mathscr{F}_{\ell, 1}$. The decomposition (2-6) (for any $\mathrm{X}$ ) shows (ext1) for any $u \in \mathcal{E}$ and enables proving that $\mathcal{E}$ satisfies all assumptions of Proposition 2.4. So, $\mathcal{E}$ constists of all Borel functions $u: \mathscr{K} \rightarrow \mathscr{M}$ that satisfy (2-2) and, consequently, (ext2) holds (again by (2-6) ), which finishes the proof.

Proof of Theorem 2.1. By Proposition 2.2, there is a $\sigma$-compact kernel $\mathscr{K}$ for $\mathscr{M}^{(\ell)}$. Put $\mathcal{E}^{\prime} \stackrel{\text { def }}{=}\left\{\left.u\right|_{\mathscr{K}}: u \in \mathcal{E}\right\}$ and similarly $\mathscr{F}_{0}^{\prime} \stackrel{\text { def }}{=}\left\{\left.u\right|_{\mathscr{K}}: u \in \mathscr{F}_{0}\right\}$, and observe that conditions (E1)-(E2) are fulfilled for $\mathcal{E}^{\prime}$ and $\mathscr{F}_{0}^{\prime}$ (by Corollary 2.5). So, Proposition 2.4 yields that $\mathcal{E}^{\prime}=\mathscr{F}_{0}^{\prime}$. Thus, we conclude from Corollary 2.5 that $\mathcal{E}=\mathscr{F}_{0}$.

The next result is an immediate consequence of Corollary 2.5. The proof is skipped.

2.6. Corollary. Let $\mathscr{F}_{0}$ denote one of $\mathscr{F}_{\ell, 1}, \mathscr{F}_{\ell, 1}^{\text {loc }}$ or $\mathscr{F}_{\ell, 1}^{\text {bd }}$. The center $\mathcal{Z}\left(\mathscr{F}_{0}\right)$ of $\mathscr{F}_{0}$ coincides with the set of all functions $u \in \mathscr{F}_{0}$ such that $u(\mathrm{X})$ is a scalar multiple of the unit matrix for any irreducible $\ell$-tuple $\mathrm{X} \in \mathscr{M}^{(\ell)}$.

In the sequel we shall also need the next two results.

2.7. Lemma. Every Borel set $\mathscr{K}_{0}$ in $\mathscr{M}_{m}^{(\ell)}$ that consists of mutually unitarily inequivalent irreducible $\ell$-tuples is contained in a Borel kernel $\mathscr{K}$ of $\mathscr{M}^{(\ell)}$.

Proof. Let $\mathscr{Z}$ be the center of $\mathscr{U}_{m}$. It follows e.g. from Theorem 1.2.4 in 2] that there is a Borel set $\mathscr{D} \subset \mathscr{U}_{m}$ which meets each coset of $\mathscr{Z}$ in exactly one point. This implies that the function $\phi: \mathscr{D} \times \mathscr{K}_{0} \ni(U, \mathrm{X}) \mapsto U . \mathrm{X} \in \mathscr{M}_{m}^{(\ell)}$ is one-to-one (because members of $\mathscr{K}_{0}$ are irreducible). So, it follows from a theorem of Suslin (see Corollary A.7 in [50] or Theorem 9 in $\S 1$ of Chapter XIII in [30]) that the image $\mathscr{B}$ of $\phi$ is a Borel set in $\mathscr{M}_{m}^{(\ell)}$. Hence, the set $\mathscr{L} \stackrel{\text { def }}{=} \mathscr{M}^{(\ell)} \backslash \mathscr{B}$ is Borel in $\mathscr{M}^{(\ell)}$. Notice that $\mathscr{L} \cap \mathscr{K}_{0}=\varnothing$ and $\mathscr{L}$ is unitarily invariant, that is, $U . \mathrm{X} \in \mathscr{L}$ for any

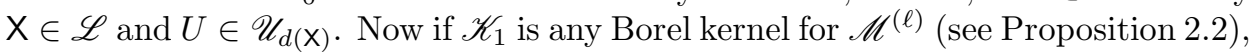
it suffices to put $\mathscr{K} \stackrel{\text { def }}{=} \mathscr{K}_{0} \cup\left(\mathscr{L} \cap \mathscr{K}_{1}\right)$ to get the kernel we searched for. 
The following result may be proved using methods and concepts of 42 . Below we give an alternative proof (especially that we shall use a part of it in the last section).

2.8. Lemma. Every Borel function $u: \mathscr{K} \rightarrow \mathscr{M}$ defined on a Borel kernel $\mathscr{K}$ of $\mathscr{M}^{(\ell)}$ and satisfying (2-2) admits a unique extension $\hat{u}$ to a function in $\mathscr{F}_{\ell, 1}$. Moreover, conditions (ext1)-(ext2) hold.

Proof. All we need to show is that $u$ admits an extension (see the proof of Corollary [2.5). Arguing as in the proof of Lemma 2.7, we see that for any $n>0$ there exists a Borel set $\mathscr{D}_{n} \subset \mathscr{U}_{n}$ which meets each coset of the center of $\mathscr{U}_{n}$ at exactly one point. We put $\mathscr{D} \stackrel{\text { def }}{=} \bigcup_{n=1}^{\infty} \mathscr{D}_{n}$. Since $\mathscr{K}$ consists of irreducible $\ell$-tuples, we see that the function $\varphi: \bigcup_{n=1}^{\infty}\left(\mathscr{D}_{n} \times\left(\mathscr{K} \cap \mathscr{M}_{n}^{\ell}\right)\right) \ni(U, \mathrm{X}) \mapsto U . \mathrm{X} \in \mathscr{M}^{(\ell)}$ is oneto-one (it is also Borel). Now let $\mathscr{K}^{\prime}$ be a $\sigma$-compact kernel of $\mathscr{M}^{(\ell)}$. Then the set $\mathscr{L} \stackrel{\text { def }}{=} \varphi^{-1}\left(\mathscr{K}^{\prime}\right)$ is Borel and the projection $\mathscr{L} \ni(U, \mathrm{X}) \mapsto \mathrm{X} \in \mathscr{K}$ onto the second coordinate is a bijection. Applying Suslin theorem (see the previous proof), we conclude that the function $g: \mathscr{K} \rightarrow \mathscr{D}$ that assigns to each $\mathrm{X} \in \mathscr{K}$ the unique unitary matrix $U \in \mathscr{D}$ such that $(U, \mathrm{X}) \in \mathscr{L}$ is Borel. Notice that then

$$
\psi: \mathscr{K} \ni \mathrm{X} \mapsto g(\mathrm{X}) . \mathrm{X} \in \mathscr{K}^{\prime}
$$

is a well defined Borel isomorphism. Now it remains to apply Corollary 2.5 to the function $u^{\prime}: \mathscr{K}^{\prime} \ni \mathrm{X} \mapsto\left(g \circ \psi^{-1}\right)(\mathrm{X}) .\left(u \circ \psi^{-1}\right)(\mathrm{X}) \in \mathscr{M}$ to obtain the extension $\hat{u} \in \mathscr{F}_{\ell, 1}$ of $u$ and then to check that $\hat{u}$ extends $u$ (which is left to the reader).

\section{Functional calculus}

This section is mainly devoted to the proof of the following

3.1. Theorem. Let $\mathcal{M}$ be a finite type I von Neumann algebra in a Hilbert space $\mathcal{H}$. For any finite tuple $\mathrm{T}=\left(T_{1}, \ldots, T_{\ell}\right)$ of operators in $\hat{\mathcal{M}}$ there exists a unique function that assigns to every function $u$ in $\mathscr{F}_{\ell, \ell^{\prime}}$ an $\ell^{\prime}$-tuple $u\left[T_{1}, \ldots, T_{\ell}\right]$ of operators that belong to $\hat{\mathcal{M}}$ in a way such that the following conditions hold:

(F1) $\pi_{j}^{(\ell)}[\mathrm{T}]=T_{j}$ for any $j \leqslant \ell$;

(F2) for any finite collection $\left\{f_{1}, \ldots, f_{\ell^{\prime}}\right\}$ of functions in $\mathscr{F}_{\ell, 1}$,

$$
\left(f_{k}\right)_{k=1}^{\ell^{\prime}}[\mathrm{T}]=\left(f_{1}[\mathrm{~T}], \ldots, f_{\ell^{\prime}}[\mathrm{T}]\right)
$$

(F3) if $f \in \mathscr{F}_{\ell, 1}^{b d}$, then $f[\mathrm{~T}]$ is bounded;

(F4) the function $\mathscr{F} \ell, 1 \ni u \mapsto u[\mathrm{~T}] \in \hat{\mathcal{M}}$ is a *-homomorphism;

(F5) whenever $u_{n} \in \mathscr{F}_{\ell, 1}^{b d}$ are uniformly bounded and converge pointwise to $u \in$ $\mathscr{F}_{\ell, 1}^{\text {bd }}$, then $u_{n}[\mathrm{~T}]$ converge to $u[\mathrm{~T}]$ in the $*$-strong operator topology of $\mathcal{B}(\mathcal{H})$.

Moreover,

(F6) $v[u[\mathrm{~T}]]=(v \circ u)[\mathrm{T}]$ for any $u \in \mathscr{F}_{\ell, \ell^{\prime}}$ and $v \in \mathscr{F}_{\ell^{\prime}, \ell^{\prime \prime}}$;

(F7) if $T_{j} \in \mathcal{M}$ for each $j$, then $u[\mathrm{~T}]$ is bounded for any $u \in \mathscr{F}_{\ell, 1}^{\text {loc }}$;

(F8) for any $u \in \mathscr{F}_{\ell, 1}, u[\mathrm{~T}] \in \hat{\mathcal{W}}$ where $\mathcal{W}=\mathcal{W}^{\prime \prime}(\mathrm{T})$;

(F9) if $\mathcal{H}$ is separable, then $\left\{u[\mathrm{~T}]: u \in \mathscr{F}_{\ell, 1}^{b d}\right\}=\mathcal{W},\left\{u[\mathrm{~T}]: u \in \mathcal{Z}\left(\mathscr{F}_{\ell, 1}^{b d}\right)\right\}=$ $\mathcal{Z}(\mathcal{W}),\left\{u[\mathrm{~T}]: u \in \mathscr{F}_{\ell, 1}\right\}=\hat{\mathcal{W}}$ and $\left\{u[\mathrm{~T}]: u \in \mathcal{Z}\left(\mathscr{F}_{\ell, 1}\right)\right\}=\mathcal{Z}(\hat{\mathcal{W}})$ (where $\left.\mathcal{W}=\mathcal{W}^{\prime \prime}(\mathrm{T})\right)$.

We precede the proof of Theorem 3.1 by a few auxiliary results.

3.2. Lemma. Let $T_{1}, \ldots, T_{\ell}$ be operators in a Hilbert space $\mathcal{H}$. The following conditions are equivalent:

(i) there is a finite type I von Neumann algebra $\mathcal{M}$ which each of $T_{j}$ is affiliated with; 
(ii) $\mathcal{W}^{\prime \prime}\left(T_{1}, \ldots, T_{\ell}\right)$ is finite and type $\mathrm{I}$.

Proof. We only need to check that (ii) is implied by (i). Observe that, when $\mathcal{M}$ is as specified in (i), $\mathcal{W}^{\prime \prime}\left(T_{1}, \ldots, T_{\ell}\right) \subset \mathcal{M}$. So, the conclusion of (ii) follows from the fact that a von Neumann subalgebra of a finite type I von Neumann algebra is also finite and type I, which may simply be deduced, e.g., from Proposition III.1.5.14 in $[3]$.

For simplicity, we introduce

3.3. Definition. An $\ell$-tuple $\mathrm{T}=\left(T_{1}, \ldots, T_{\ell}\right)$ of operators in a Hilbert space $\mathcal{H}$ is said to be $\mathrm{FTI}$ if $\mathcal{W}^{\prime \prime}(\mathrm{T})$ is finite and type I. For every unitary operator $U: \mathcal{H} \rightarrow \mathcal{K}$ (between Hilbert spaces) we use $U . T$ to denote the $\ell$-tuple $\left(U T_{1} U^{-1}, \ldots, U T_{\ell} U^{-1}\right)$ of operators in $\mathcal{K}$. A closed linear subspace $\mathcal{E}$ of $\mathcal{H}$ is reducing for $\mathrm{T}$ if $\mathcal{E}$ reduces each of $T_{j}$. If this happens, $\left.\mathrm{T}\right|_{\mathcal{E}}$ is defined as the tuple $\left(\left.T_{1}\right|_{\mathcal{E}}, \ldots,\left.T_{\ell}\right|_{\mathcal{E}}\right)$ of operators in $\mathcal{E}$. Direct sums of $\ell$-tuples of operators are defined coordinatewise; that is, if, for $s \in S, \mathrm{~T}^{(s)}=\left(T_{1}^{(s)}, \ldots, T_{\ell}^{(s)}\right)$, then $\bigoplus_{s \in S} \mathrm{~T}^{(s)}$ denotes the $\ell$-tuple $\left(\bigoplus_{s \in S} T_{1}^{(s)}, \ldots, \bigoplus_{s \in S} T_{\ell}^{(s)}\right)$. Finally, if each of $T_{j}$ is bounded, we use $\|\mathrm{T}\|$ to denote the maximum of $\left\|T_{j}\right\|$; otherwise we put $\|\mathrm{T}\| \stackrel{\text { def }}{=} \infty$.

According to the terminology of [37, the next result asserts that FTI $\ell$-tuples form an ideal. Its proof is given in 39 .

3.4. Lemma. Let $\mathrm{T}$ be an $\mathrm{FTI} \ell$-tuple of operators in a Hilbert space $\mathcal{H}$.

(A) If $U: \mathcal{H} \rightarrow \mathcal{K}$ is unitary, then $U . \mathrm{T}$ is $\mathrm{FTI}$.

(B) If $\mathcal{E} \subset \mathcal{H}$ is a (nonzero) reducing subspace for $\mathrm{T}$, then $\left.\mathrm{T}\right|_{\mathcal{E}}$ is $\mathrm{FTI}$.

(C) The direct sum of any collection of $\mathrm{FTI} \ell$-tuples is $\mathrm{FTI}$ as well.

3.5. Lemma. Let $\mathrm{T}$ be an $\ell$-tuple of bounded operators acting on a separable Hilbert space such that $\mathcal{W}(\mathrm{T})$ is type $\mathrm{I}_{m}$ (with finite $m$ ), $\mathcal{W}^{\prime}(\mathrm{T})$ is commutative and $\|\mathrm{T}\|=$ $R$. Then there exist a unital $*$-homomorphism $\mathscr{F}_{\ell, 1}^{\text {loc }} \ni u \mapsto u[\mathrm{~T}] \in \mathcal{W}(\mathrm{T})$ and a probabilistic Borel measure $\lambda$ on $\mathscr{M}^{(\ell)}$ such that (F1) holds and

$(\Lambda 0) \lambda$ is concentrated on the set $\mathscr{S}$ of all irreducible $\ell$-tuples in $\mathscr{M}_{m}^{\ell} \cap \mathscr{B}^{(\ell)}(R)$ and for any $u, v \in \mathscr{F}_{\ell, 1}^{\text {loc }}, u[\mathrm{~T}]=v[\mathrm{~T}]$ iff $u$ and $v$ are equal $\lambda$-almost everywhere; and

$(\Lambda 1)$ whenever $u_{n} \in \mathscr{F}_{\ell, 1}^{\text {loc }}$ are uniformly bounded on $\mathscr{S}$ and converge pointwise $\lambda$ almost everywhere to $u \in \mathscr{F}_{\ell, 1}^{\text {loc }}$, then $u_{n}[\mathrm{~T}]$ converge to $u[\mathrm{~T}]$ in the $*$-strong operator topology; and

$(\Lambda 2)$ if functions $u_{n} \in \mathscr{F}_{\ell, 1}^{\text {loc }}$ are uniformly bounded on $\mathscr{S}$ and $u_{n}[\mathrm{~T}]$ converge pointwise to 0 , then there is a subsequence $\left(u_{\nu_{n}}\right)_{n=1}^{\infty}$ of $\left(u_{n}\right)_{n=1}^{\infty}$ such that the functions $u_{\nu_{n}}$ converge pointwise $\lambda$-almost everywhere to the zero function in $\mathscr{F}_{\ell, 1}^{\text {loc }}$; and

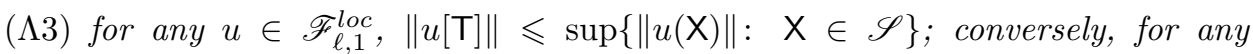
$S \in \mathcal{W}(\mathrm{T})($ resp. $S \in \mathcal{Z}(\mathcal{W}(\mathrm{T})))$ there exists $u \in \mathscr{F}_{\ell, 1}^{b d}$ (resp. $\left.u \in \mathcal{Z}\left(\mathscr{F}_{\ell, 1}^{b d}\right)\right)$ with $u[\mathrm{~T}]=S$ and $\|u\| \leqslant\|S\|$.

Proof. Since $\mathcal{W}(T)$ is a type $I_{m}$ von Neumann algebra acting on a separable Hilbert space (say $\mathcal{H}$ ) and $\mathcal{W}^{\prime}(\mathrm{T})$ is commutative, it follows from reduction theory due to von Neumann [53] (see also Chapter 14 in [26], 33.2 in [48], $\S 8$ of Chapter IV in [50]) that there exist a standard Borel space $(\Omega, \mathfrak{M})$, a probabilistic measure $\mu: \mathfrak{M} \rightarrow[0,1]$ and a unitary operator $U: \mathcal{H} \rightarrow L^{2}\left(\Omega, \mu, \mathbb{C}^{m}\right)$ such that the algebra $\left\{U S U^{-1}: S \in \mathcal{W}(\mathrm{T})\right\}$ coincides with the set of all bounded decomposable operators on $L^{2}\left(\Omega, \mu, \mathbb{C}^{m}\right)$. Recall that $L^{2}\left(\Omega, \mu, \mathbb{C}^{m}\right)$ is the Hilbert space of all measurable functions $f: \Omega \rightarrow \mathbb{C}^{m}$ for which $\left(\|f\|^{2}=\right) \int_{\Omega}\|f(\omega)\|^{2} \mathrm{~d} \mu(\omega)<\infty$, and a bounded 
operator $S$ on $L^{2}\left(\Omega, \mu, \mathbb{C}^{m}\right)$ is decomposable if there is a bounded measurable function $u: \Omega \rightarrow \mathscr{M}_{m}$ such that $S=M_{u}$ where $\left(M_{u} f\right)(\omega) \stackrel{\text { def }}{=} u(\omega) f(\omega)$ for each $\omega \in \Omega$. The proof of Theorem 14.1.10 in [26] shows that

(S) if $w_{n}: \Omega \rightarrow \mathscr{M}_{m}$ are uniformly bounded measurable functions such that $M_{w_{n}}$ converge to $M_{w}$ in the strong operator topology, then there exists a subsequence $\left(w_{\nu_{n}}\right)_{n=1}^{\infty}$ of $\left(w_{n}\right)_{n=1}^{\infty}$ such that the functions $w_{\nu_{n}}$ converge pointwise $\mu$-almost everywhere to $w$.

Now write $\mathrm{T}=\left(T_{1}, \ldots, T_{\ell}\right)$ and denote by $\xi_{j}: \Omega \rightarrow \mathscr{M}_{n}$ a bounded measurable function such that $U T_{j} U^{-1}=M_{\xi_{j}}$. We may and do assume that

$$
\left\|\xi_{j}\right\| \leqslant R
$$

for each $j$. We claim that there exists a set $Z \in \mathfrak{M}$ such that $\mu(Z)=0$ and

(\$) the function

$$
\psi: \Omega \backslash Z \ni \omega \mapsto\left(\xi_{1}(\omega), \ldots, \xi_{\ell}(\omega)\right) \in \mathscr{M}_{n}^{\ell}
$$

is one-to-one and its range $\mathscr{K}_{0}$ is contained in $\mathscr{S}$ and consists of mutually unitarily inequivalent irreducible $\ell$-tuples.

This property is well-known, but, for the reader's convenience, we give its proof, which is similar to that of item 2 of Theorem 3.4 in [15]. Since $\Omega$ is standard, there is a sequence $v_{1}, v_{2}, \ldots: \Omega \rightarrow \mathscr{M}_{m}$ of bounded measurable functions such that

(a) for any two distinct points $a$ and $b$ of $\Omega$, the set $\left\{\left(v_{j}(a), v_{j}(b)\right): j>0\right\}$ is dense in $\mathscr{M}_{m} \times \mathscr{M}_{m}$.

Further, we conclude from the property that $\mathcal{W}\left(M_{\xi_{1}}, \ldots, M_{\xi_{\ell}}\right)$ contains each of $M_{v_{n}}$, Kaplansky's density theorem and property $(\mathrm{S})$ that for any $j$ there are a sequence $p_{1}^{(j)}, p_{2}^{(j)}, \ldots$ of polynomials in $2 \ell$ noncommuting variables and a set $Z_{j} \in \mathfrak{M}$ such that $\mu\left(Z_{j}\right)=0$ and

$$
\lim _{n \rightarrow \infty} p_{n}^{(j)}\left(\xi_{1}(\omega), \ldots, \xi_{\ell}(\omega), \xi_{1}^{*}(\omega), \ldots, \xi_{\ell}^{*}(\omega)\right)=v_{j}(\omega)
$$

for any $\omega \in \Omega \backslash Z_{j}$. We put $Z \stackrel{\text { def }}{=} \bigcup_{j=1}^{\infty} Z_{j}$. Notice that $\mu(Z)=0$ and (3-3) holds for any $\omega \in \Omega \backslash Z$, which implies that $\psi$, given by (3-2), is one-to-one (because the functions $v_{n}$ separate points of $\Omega$, by (*)). Moreover, if $\omega, \omega^{\prime} \in \Omega \backslash Z$ and $W \in \mathscr{U}_{m}$ are such that $W \cdot \psi(\omega)=\psi\left(\omega^{\prime}\right)$, then $W \cdot v_{j}(\omega)=v_{j}\left(\omega^{\prime}\right)$ for any $j$ (again by (3-3)) and hence $\omega=\omega^{\prime}$ (thanks to $(\boldsymbol{*})$ ). Further, it follows from (3-1) that the range $\mathscr{K}_{0}$ of $\psi$ is contained in $\mathscr{M}_{m}^{\ell} \cap \mathscr{B}^{(\ell)}(R)$ and therefore it remains to check that each value of $\psi$ is an irreducible $\ell$-tuple. But this again follows from (3-3) and ( $)$, because the former formula implies that $\mathcal{W}(\psi(\omega))$ coincides with $\mathscr{M}_{m}$ for any $\omega \notin Z$. So, the proof of $(\boldsymbol{\omega})$ is complete.

Replacing $\Omega$ by $\Omega \backslash Z$, we may and do assume that $Z=\varnothing$. Further, since $\Omega$ and $\mathscr{M}_{m}^{\ell}$ are standard measure spaces and $\psi$ is a one-to-one measurable function, we conclude that $\mathscr{K}_{0}$ is a Borel subset of $\mathscr{M}_{m}^{\ell}$ and $\psi$ is a Borel isomorphism of $\Omega$ onto $\mathscr{K}_{0}$ (consult, for example, Corollary A.7 in [50] or Theorem 9 in $\S 1$ of Chapter XIII in [30]). So, it follows from Lemma 2.7 that there is a Borel kernel $\mathscr{K}$ of $\mathscr{M}^{(\ell)}$ which contains $\mathscr{K}_{0}$. We now define a probabilistic measure $\lambda: \mathfrak{B}\left(\mathscr{M}^{(\ell)}\right) \rightarrow[0,1]$ as the transport of $\mu$ under $\psi$; that is, $\lambda(\mathscr{B}) \stackrel{\text { def }}{=} \mu\left(\psi^{-1}(\mathscr{B})\right)$ for any Borel set $\mathscr{B}$ in $\mathscr{M}^{(\ell)}$. We see that $\lambda\left(\mathscr{K}_{0}\right)=1$ and therefore the first claim of $(\Lambda 0)$ holds (see $(\boldsymbol{\uparrow})$ ). Finally, for each $u \in \mathscr{F}_{\ell, 1}^{\text {loc }}$, we define $u[\mathrm{~T}]$ by $u[\mathrm{~T}] \stackrel{\text { def }}{=} U^{-1} M_{u \circ \psi} U(\in \mathcal{W}(\mathrm{T}))$. It is readily seen that the assignment $u \mapsto u[\mathrm{~T}]$ correctly defines a unital $*$-homomorphism for which (F1), the second claim of $(\Lambda 1)$ and the first of $(\Lambda 3)$ hold (recall that $\pi_{j}^{(\ell)} \circ \psi=\xi_{j}$ ). Furthermore, for any $S \in \mathcal{W}(\mathrm{T})$ (resp. $S \in \mathcal{Z}(\mathcal{W}(\mathrm{T}))$ ) there is a bounded Borel function $v: \Omega \rightarrow \mathscr{M}_{m}$ (resp. $\left.v: \Omega \rightarrow \mathbb{C} \cdot I_{m}\right)$ such that $U S U^{-1}=M_{v}$ 
and $\|v\| \leqslant\|S\|$. Then let $u_{0}: \mathscr{K} \rightarrow \mathscr{M}$ coincide with $v \circ \psi^{-1}$ on $\mathscr{K}_{0}$ and vanish at each point of $\mathscr{K} \backslash \mathscr{K}_{0}$. We then infer from Lemma 2.8 (and Corollary 2.6) that there is $u \in \mathscr{F}_{\ell, 1}^{b d}$ (resp. $\left.u \in \mathcal{Z}\left(\mathscr{F}_{\ell, 1}^{b d}\right)\right)$ which extends $u_{0}$ and satisfies $\|u\| \leqslant\|S\|$. This implies that $u \circ \psi=v$ and hence $u[\mathrm{~T}]=M_{v}$. So, the whole assertion of $(\Lambda 3)$ holds and thus it remains to verify conditions $(\Lambda 1)$ and $(\Lambda 2)$.

If $u_{n}$ and $u$ are as specified in $(\Lambda 1)$, then the functions $u_{n} \circ \psi$ are uniformly bounded and converge pointwise $\mu$-almost everywhere to $u \circ \psi$ (by the definition of $\lambda)$. Then for each $g \in L^{2}\left(\Omega, \mu, \mathbb{C}^{m}\right)$ we have $\left\|u_{n}(\psi(\omega)) g(\omega)\right\|^{2} \leqslant C\|g(\omega)\|^{2}$ for each $n$ and almost all $\omega \in \Omega$ (where $C$ is a positive contant independent of $n$ ) and therefore, by Lebesgue's dominated convergence theorem, $\lim _{n \rightarrow \infty} \int_{\Omega} \| u_{n}(\psi(\omega)) g(\omega)-$ $u(\psi(\omega)) g(\omega) \|^{2} \mathrm{~d} \mu(\omega)=0$. This shows that the operators $M_{u_{n} \circ \psi}$ converge to $M_{u \circ \psi}$ in the strong operator topology. Consequently, $u_{n}[\mathrm{~T}]$ converge so to $u[\mathrm{~T}]$. But also the functions $u_{n}^{*}$ are uniformly bounded on $\mathscr{S}$ and converge pointwise $\lambda$-almost everywhere to $u^{*}$. We thus conclude that $u_{n}^{*}[\mathrm{~T}]$ converge pointwise to $u[\mathrm{~T}]$, which finishes the proof of $(\Lambda 1)$.

Finally, assume $u_{n}$ are as specified in $(\Lambda 2)$. Then the functions $u_{n} \circ \psi$ are uniformly bounded and $M_{u_{n} \circ \psi}$ converge pointwise to 0 . Hence, we infer from (S) that there are a subsequence $\left(u_{\nu_{n}}\right)_{n=1}^{\infty}$ of $\left(u_{n}\right)_{n=1}^{\infty}$ and a set $B \in \mathfrak{M}$ such that $\mu(B)=1$ and $\lim _{n \rightarrow \infty} u_{\nu_{n}}(\psi(\omega))=0$ for all $\omega \in B$. Then the set $\mathscr{B} \stackrel{\text { def }}{=} \psi(B)$ is Borel in $\mathscr{M}^{(\ell)}, \lambda(\mathscr{B})=1$ and $\lim _{n \rightarrow \infty} u_{n}(\mathrm{X})=0$ for any $\mathrm{X} \in \mathscr{B}$.

Now we shall easily prove a generalization of Lemma 3.5

3.6. Proposition. Let $\mathrm{T}$ be an $\mathrm{FTI} \ell$-tuple of bounded operators acting on a separable Hilbert space with $\|\mathrm{T}\|=R$. Then there exist a unital $*$-homomorphism $\mathscr{F}_{\ell, 1}^{l o c} \ni u \mapsto u[\mathrm{~T}] \in \mathcal{W}(\mathrm{T})$ and a probabilistic Borel measure $\lambda$ on $\mathscr{M}^{(\ell)}$ such that

$\left(\Lambda 0^{\prime}\right) \lambda$ is concentrated on the set $\mathscr{S}$ of all irreducible $\ell$-tuples in $\mathscr{B}^{(\ell)}(R)$, and for any $u, v \in \mathscr{F}_{\ell, 1}^{\text {loc }}, u[\mathrm{~T}]=v[\mathrm{~T}]$ iff $u$ and $v$ are equal $\lambda$-almost everywhere and conditions $(\mathrm{F} 1)$ as well as $(\Lambda 1)-(\Lambda 3)$ hold.

Proof. Assume $\mathrm{T}$ acts on $\mathcal{H}$. There is a sequence $\mathcal{H}_{1}, \mathcal{H}_{2}, \ldots$ (finite or not) of reducing subspaces for $\mathrm{T}$ such that $\mathcal{H}=\bigoplus_{n \geqslant 1} \mathcal{H}_{n}, \mathcal{W}\left(\mathrm{T}^{(n)}\right.$ ) is type $\mathrm{I}_{p_{n}}$ (for some $p_{n}>0$ ) and $\mathcal{W}^{\prime}\left(\mathrm{T}^{(n)}\right)$ is commutative for any $n$ where $\left.\mathrm{T}^{(n)} \stackrel{\text { def }}{=} \mathrm{T}\right|_{\mathcal{H}_{n}}$ (to convince oneself that such a decomposition exists, consult, for example, Theorem 3.6.1 in 37]). Now to each of $\mathrm{T}^{(n)}$ we apply Lemma 3.5 to obtain a respective probabilistic

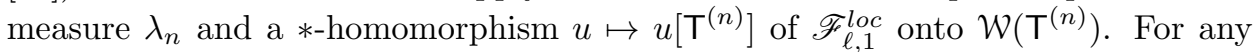
$u \in \mathscr{F}_{\ell, 1}^{l o c}$ we now put $u[\mathrm{~T}] \stackrel{\text { def }}{=} \bigoplus_{n \geqslant 1} u\left[\mathrm{~T}^{(n)}\right]$. We also put $\lambda \stackrel{\text { def }}{=} \sum_{n=1}^{\infty} 2^{-n} \lambda_{n}$. We see that conditions $\left(\Lambda 0^{\prime}\right)$ and $(\Lambda 1)$ as well as the first claim of $(\Lambda 3)$ hold (and thus $u[\mathrm{~T}]$ is a bounded operator). It is also readily seen that the assignment $u \mapsto u[\mathrm{~T}]$ correctly defines a unital $*$-homomorphism of $\mathscr{F}_{\ell, 1}^{\text {loc }}$ into $\mathcal{B}(\mathcal{H})$. To conclude that in fact $u[\mathrm{~T}] \in \mathcal{W}(\mathrm{T})$ for any $u$, we employ Theorem 2.1 the family $\mathcal{E} \stackrel{\text { def }}{=}\{u \in$

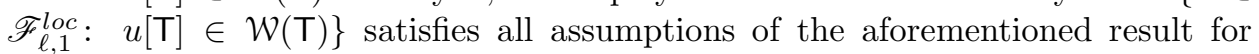
$\mathscr{F}_{0}=\mathscr{F}_{\ell, 1}^{\text {loc }}$ (thanks to $(\Lambda 1)$ ), and hence $\mathcal{E}=\mathscr{F}_{\ell, 1}^{\text {loc }}$.

We turn to $(\Lambda 2)$. Assume $u_{n}$ are as specified there. Then $u_{n}\left[\mathrm{~T}^{(m)}\right]$ converge to 0 in the strong operator topology (when $n$ tends to $\infty$ ) for each fixed $m$. So, using the diagonal argument, we conclude that there is a subsequence $\left(u_{\nu_{n}}\right)_{n=1}^{\infty}$ such that the functions $u_{\nu_{n}}$ converge pointwise $\lambda_{k}$-almost everywhere to the zero function in $\mathscr{F}_{\ell, 1}^{\text {loc }}$ for each $k$. Since the set $\mathscr{B}_{0} \stackrel{\text { def }}{=}\left\{\mathrm{X} \in \mathscr{M}^{(\ell)}: \lim _{n \rightarrow \infty}\left\|u_{\nu_{n}}(\mathrm{X})\right\|=0\right\}$ is Borel, we see from the definition of $\lambda$ that $\lambda\left(\mathscr{B}_{0}\right)=1$, which proves $(\Lambda 2)$.

Finally, we turn to the remainder of $(\Lambda 3)$. To this end, let $S \in \mathcal{W}(\mathrm{T})$ (resp. $S \in$ $\mathcal{Z}(\mathcal{W}(\mathrm{T})))$. It follows from Kaplansky's density theorem that there are polynomials 
$p_{n} \in \mathscr{F}_{\ell, 1}^{l o c}$ in $2 \ell$ noncommuting variables such that the operators $p_{n}[\mathrm{~T}]$ converge to $S$ in the strong operator topology. Then (by $(\Lambda 3)$ for $\mathrm{T}^{(k)}$ ) for any $k$ there is a function $u_{k} \in \mathscr{F}_{\ell, 1}^{b d}$ (resp. $u_{k} \in \mathcal{Z}\left(\mathscr{F}_{\ell, 1}^{b d}\right)$ ) such that $\left\|u_{k}\right\| \leqslant\|S\|$ and

$$
\lim _{n \rightarrow \infty}\left(p_{n}\left[\mathbf{T}^{(k)}\right]-u_{k}\left[\mathbf{T}^{(k)}\right]\right)=0 .
$$

We now employ $(\Lambda 2)$ for each $k$. Using again the diagonal argument and passing to a subsequence, we may assume that the matrix functions $p_{n}$ converge pointwise $\lambda_{k}$-almost everywhere to $u_{k}$ for any $k$. Let $\mathscr{D}$ consist of all $\ell$-tuples $\mathrm{X} \in \mathscr{M}^{(\ell)}$ for which the sequence $\left(p_{n}(\mathbf{X})\right)_{n=1}^{\infty}$ is convergent to a matrix (resp. to a scalar multiple of the unit matrix) whose norm does not exceed $\|S\|$. Then $\mathscr{D} \in \mathfrak{B}\left(\mathscr{M}^{(\ell)}\right)$ and $\lambda_{k}(\mathscr{D})=1$. Consequently, $\lambda(\mathscr{D})=1$ as well. Let $u^{\prime}: \mathscr{D} \rightarrow \mathscr{M}$ be the pointwise limit of the polynomials $p_{n}$. We claim that there is $u \in \mathscr{F}_{\ell, 1}^{b d}\left(\right.$ resp. $\left.u \in \mathcal{Z}\left(\mathscr{F}_{\ell, 1}^{b d}\right)\right)$ that extends $u^{\prime}$ and has the same norm as $u^{\prime}$. Assume we have such a function $u$. Then $u$ and $u_{k}$ are equal $\lambda_{k}$-almost everywhere and thus $u\left[\mathbf{T}^{(k)}\right]=u_{k}\left[\mathbf{T}^{(k)}\right]$ (by $(\Lambda 0)$ ). So, $u\left[\mathbf{T}^{(k)}\right]$ is the limit of $p_{n}\left[\mathbf{T}^{(k)}\right]$ in the strong operator topology. Consequently, $u[\mathrm{~T}]=\bigoplus_{k=1}^{\infty} u\left[\mathrm{~T}^{(k)}\right]$ is the limit of $p_{n}[\mathrm{~T}]$ and hence $S=u[\mathrm{~T}]$, which completes the proof of $(\Lambda 3)$. So, we see it is enough to show the existence of $u$.

First note that $\left\|u^{\prime}\right\| \leqslant\|S\|$, by the very definition of $\mathscr{D}$. Take a $\sigma$-compact kernel $\mathscr{K}$ of $\mathscr{M}^{(\ell)}$ and observe that:

$(\mathscr{D} 1)$ if $\mathrm{X}, \mathrm{Y} \in \mathscr{M}^{(\ell)}$ are such that $\mathrm{X} \oplus \mathrm{Y} \in \mathscr{D}$, then both $\mathrm{X}$ and $\mathrm{Y}$ belong to $\mathscr{D}$; and $(\mathscr{D} 2)$ whenever $\mathrm{X}_{1}, \ldots, \mathrm{X}_{s}$ belong to $\mathscr{D}$ and $V$ belongs to $\mathscr{U}_{N}$ with $N=\sum_{j=1}^{s} d\left(\mathrm{X}_{j}\right)$, then $V \cdot\left(\bigoplus_{j=1}^{s} \mathrm{X}_{j}\right) \in \mathscr{D}$ and $u^{\prime}\left(V \cdot\left(\bigoplus_{j=1}^{s} \mathrm{X}_{j}\right)\right)=V \cdot\left(\bigoplus_{j=1}^{s} u^{\prime}\left(\mathrm{X}_{j}\right)\right)$.

These two properties imply that for any $\mathrm{X} \in \mathscr{D}$ there are $V \in \mathscr{U}_{d(\mathrm{X})}$ and some $\mathrm{X}_{1}, \ldots, \mathrm{X}_{p} \in \mathscr{K} \cap \mathscr{D}$ with $\mathrm{X}=V \cdot\left(\bigoplus_{j=1}^{p} \mathrm{X}_{j}\right)$. Now let $u_{0}: \mathscr{K} \rightarrow \mathscr{M}$ be a function that coincides with $u^{\prime}$ on $\mathscr{K} \cap \mathscr{D}$ and vanishes at each point of $\mathscr{K} \backslash \mathscr{D}$. It follows from Corollary 2.5 (and Corollary 2.6) that there is $u \in \mathscr{F}_{\ell, 1}^{b d}$ (resp. $u \in \mathcal{Z}\left(\mathscr{F}_{\ell, 1}^{b d}\right)$ ) which extends $u_{0}$ and has the same norm as $u_{0}$. Then automatically $u$ extends $u^{\prime}$ (by $(\mathscr{D} 2)$ ) and $\|u\|=\left\|u^{\prime}\right\|$.

3.7. Remark. It follows from Proposition 3.6 that for any FTI $\ell$-tuple $\mathrm{T}$ of bounded operators on a separable Hilbert space, there exists a probabilistic Borel measure $\lambda$ on $\mathscr{M}^{(\ell)}$ such that the assignment $u \mapsto u[\mathrm{~T}]$ defines a $*$-isomorphism of $L_{c m}^{\infty}(\lambda)$ onto $\mathcal{W}(\mathrm{T})$ where $L_{c m}^{\infty}(\lambda)$ is the quotient $C^{*}$-algebra $\mathscr{F}_{\ell, 1}^{b d} / \lambda$ consisting of all equivalence classes of (all) bounded compatible Borel functions from $\mathscr{M}^{(\ell)}$ into $\mathscr{M}$ with respect to $\lambda$-almost everywhere equality.

3.8. Theorem. Let $\mathrm{T}$ be an $\mathrm{FTI} \ell$-tuple of bounded operators on a Hilbert space $\mathcal{H}$. There exists a unique unital $*$-homomorphism $\mathscr{F}_{\ell, 1}^{\text {loc }} \ni u \mapsto u[\mathrm{~T}] \in \mathcal{B}(\mathcal{H})$ such that (F1) holds and

$\left(\Lambda 1^{\prime}\right)$ whenever $u_{n} \in \mathscr{F}_{\ell, 1}^{\text {loc }}$ are uniformly bounded on $\mathscr{B}^{(\ell)}(\|\mathrm{T}\|)$ and converge pointwise to $u \in \mathscr{F}_{\ell, 1}^{\text {loc }}$, then $u_{n}[\mathrm{~T}]$ converge to $u[\mathrm{~T}]$ in the $*$-strong operator topology.

Moreover,

$\left(\Lambda 3^{\prime}\right)\|u[\mathrm{~T}]\| \leqslant\left\{\|u(\mathrm{X})\|: \quad \mathrm{X} \in \mathscr{B}^{(\ell)}(\|\mathrm{T}\|)\right\}$ and $u[\mathrm{~T}] \in \mathcal{W}(\mathrm{T})$ for any $u \in \mathscr{F}_{\ell, 1}^{\text {loc }}$; and $v[\mathrm{~T}] \in \mathcal{Z}(\mathcal{W}(\mathrm{T}))$ for each $v \in \mathcal{Z}\left(\mathscr{F}_{\ell, 1}^{\text {loc }}\right)$.

Proof. It may readily be shown (and follows from Theorem 2.2.4 in [37]) that there is a collection $\left\{\mathcal{H}_{s}\right\}_{s \in S}$ of separable reducing subspaces for $\mathrm{T}$ such that $\mathcal{H}=\bigoplus_{s \in S} \mathcal{H}_{s}$. Then each of $\left.\mathrm{T}^{(s)} \stackrel{\text { def }}{=} \mathrm{T}\right|_{\mathcal{H}_{s}}$ is FTI as well (see Lemma 3.4) and therefore we have, by Proposition 3.6. respective unital $*$-homomorphisms $u \mapsto u\left[\mathrm{~T}^{(s)}\right]$. For any $u \in \mathscr{F}_{\ell, 1}^{\text {loc }}$ 
we put $u[\mathbf{T}] \stackrel{\text { def }}{=} \bigoplus_{s \in S} u\left[\mathbf{T}^{(s)}\right]$. Condition $(\Lambda 3)$ (for each of $\mathrm{T}^{(s)}$ ) implies that $u[\mathrm{~T}]$ is bounded and the estimation for the norm of $u[\mathrm{~T}]$ specified in $\left(\Lambda 3^{\prime}\right)$ holds. It is also immediate that the assignment $u \mapsto u[\mathrm{~T}]$ correctly defines a unital $*$-homomorphism for which (F1) and $\left(\Lambda 1^{\prime}\right)$ hold. To show its uniqueness and that $u[T] \in \mathcal{W}(\mathrm{T})$ for any $u \in \mathscr{F}_{\ell, 1}^{\text {loc }}$, we employ Theorem 2.1 with $\mathscr{F}_{0}=\mathscr{F}_{\ell, 1}^{\text {loc }}$. To this end, assume $\Phi: \mathscr{F}_{\ell, 1}^{\text {loc }} \rightarrow \mathcal{B}(\mathcal{H})$ is a unital $*$-homomorphism for which respective conditions (F1) and (F5) hold and put $\mathcal{E} \stackrel{\text { def }}{=}\left\{u \in \mathscr{F}_{\ell, 1}^{\text {loc }}: \Phi(u)=u[\mathrm{~T}] \in \mathcal{W}(\mathrm{T})\right\}$. It is readily seen that $\mathcal{E}$ is a unital $*$-subalgebra of $\mathscr{F}_{\ell, 1}^{\text {loc }}$ which satisfies conditions (E0)-(E2) (mainly thanks to $\left(\Lambda 1^{\prime}\right)$ and $\left.(\mathrm{F} 5)\right)$ and therefore $\mathcal{E}=\mathscr{F}_{\ell, 1}^{\text {loc }}$, by Theorem 2.1. Finally, if $v \in$ $\mathcal{Z}\left(\mathscr{F}_{\ell, 1}^{\text {loc }}\right)$, then $v$ commutes with each of $\pi_{1}^{(\ell)},\left(\pi_{1}^{(\ell)}\right)^{*}, \ldots, \pi_{\ell}^{(\ell)},\left(\pi_{\ell}^{(\ell)}\right)^{*}$ and hence $v[\mathrm{~T}]$ commutes with each of $T_{1}, T_{1}^{*}, \ldots, T_{\ell}, T_{\ell}^{*}$ (where $\left.\left(T_{1}, \ldots, T_{\ell}\right)=\mathrm{T}\right)$. Consequently, $v[\mathrm{~T}] \in \mathcal{W}(\mathrm{T}) \cap \mathcal{W}^{\prime}(\mathrm{T})=\mathcal{Z}(\mathrm{T})$ and we are done.

Now we want to extend the functional calculus built in Theorem 3.8 to all functions in $\mathscr{F}_{\ell, 1}$. We shall do this with the aid of the next two results. The first of them is very simple and was established in [40. To simplify its statement, let us recall a suggestive notation introduced there. Whenever $\mathcal{M}$ is a fnite type I von Neumann algebra acting on $\mathcal{H},\left\{Z_{j}\right\}_{j \in J}$ is a countable collection of mutually orthogonal projections in $\mathcal{Z}(\mathcal{M})$ that sum up to the unit of $\mathcal{M}$ (in the strong operator topology) and $\left\{S_{j}\right\}_{j \in J}$ is a collection of operators in $\mathcal{M}$, a (possibly unbounded) operator $T \stackrel{\text { def }}{=} \sum_{j \in J} S_{j} Z_{j}$ is defined as follows. The domain $\mathcal{D}$ of $T$ consists of all vectors $x \in \mathcal{H}$ such that $\sum_{j \in J}\left\|S_{j} Z_{j} x\right\|^{2}<\infty$, and for each $x \in \mathcal{D}$ we put

$$
T x \stackrel{\text { def }}{=} \sum_{j \in J} S_{j} Z_{j} x .
$$

Note that the ranges of $S_{j} Z_{j}$ are mutually orthogonal and thus all summands of the series appearing in (3-4) are mutually orthogonal as well.

3.9. Lemma ([40]). Let $\mathcal{M}$ be a finite type I von Neumann algebra and $\left\{Z_{j}\right\}_{j \in J}$ be a collection of mutually orthogonal projections in $\mathcal{Z}(\mathcal{M})$ that sum up to the unit of $\mathcal{M}$.

(A) For any collection $\left\{S_{j}\right\}_{j \in J}$ of operators in $\mathcal{M}$, the operator $\sum_{j \in J} S_{j} Z_{j}$ is closed, densely defined and affiliated with $\mathcal{M}$; and $\left(\sum_{j \in J} S_{j} Z_{j}\right)^{*}=\sum_{j \in J} S_{j}^{*} Z_{j}$.

(B) For any two collections $\left\{S_{j}\right\}_{j \in J}$ and $\left\{T_{j}\right\}_{j \in J}$ of operators in $\mathcal{M}$,

$$
\begin{aligned}
\sum_{j \in J}\left(S_{j}+T_{j}\right) Z_{j} & =\left(\sum_{j \in J} S_{j} Z_{j}\right)+\left(\sum_{j \in J} T_{j} Z_{j}\right) \quad \text { and } \\
\sum_{j \in J}\left(S_{j} T_{j}\right) Z_{j} & =\left(\sum_{j \in J} S_{j} Z_{j}\right) \cdot\left(\sum_{j \in J} T_{j} Z_{j}\right)
\end{aligned}
$$

(where the operations on the right-hand sides are those in $\hat{\mathcal{M}}$ ).

For simplicity, let us call two functions $u$ and $v$ in $\mathscr{F}_{\ell, 1}$ disjoint if for any irreducible $\ell$-tuple $\mathrm{X} \in \mathscr{M}^{(\ell)}$, at least one of $u(\mathrm{X})$ and $v(\mathrm{X})$ is zero.

3.10. Lemma. Let $\mathrm{T}$ be an $\mathrm{FTI} \ell$-tuple of bounded operators on a Hilbert space $\mathcal{H}$.

(A) If $\left\{b_{j}\right\}_{j \in J}$ is a countable collection of mutually disjoint functions in $\mathscr{F}_{\ell, 1}^{b d}$ and $\sum_{j \in J} b_{j}(\mathrm{X})=I_{d(\mathrm{X})}$ for each irreducible $\mathrm{X} \in \mathscr{M}^{(\ell)}$, then $\left\{b_{j}[\mathrm{~T}]\right\}_{j \in J}$ is a collection of mutually orthogonal projections from $\mathcal{Z}(\mathcal{W}(\mathrm{T}))$ that sum up to the unit of $\mathcal{W}(\mathrm{T})$.

(B) Let $\left\{b_{j}\right\}_{j \in J}$ and $\left\{b_{s}^{\prime}\right\}_{s \in S}$ be two countable collections of mutually disjoint functions in $\mathscr{F}_{\ell, 1}^{b d}$ such that $\sum_{j \in J} b_{j}(\mathrm{X})=\sum_{s \in S} b_{s}^{\prime}(\mathrm{X})=I_{d(\mathrm{X})}$ for any irreducible 
$\mathrm{X} \in \mathscr{M}^{(\ell)}$. If $\left\{u_{j}\right\}_{j \in J}$ and $\left\{u_{s}^{\prime}\right\}_{s \in S}$ are two collections of functions in $\mathscr{F}_{\ell, 1}^{b d}$ such that

$$
\sum_{j \in J} u_{j}(\mathbf{X}) b_{j}(\mathbf{X})=\sum_{s \in S} u_{s}^{\prime}(\mathbf{X}) b_{s}^{\prime}(\mathbf{X})
$$

for any irreducible $\mathbf{X} \in \mathscr{M}^{(\ell)}$, then $\sum_{j \in J} u_{j}[\mathrm{~T}] b_{j}[\mathrm{~T}]$ and $\sum_{s \in S} u_{s}^{\prime}[\mathrm{T}] b_{s}^{\prime}[\mathrm{T}]$ coincide and are affiliated with $\mathcal{W}(\mathrm{T})$.

Proof. We start from (A). Observe that under the assumptions of (A), $b_{j}(\mathrm{X}) \in$ $\left\{0, I_{d(\mathrm{X})}\right\}$ for any $j \in J$ and irreducible $\mathrm{X} \in \mathscr{M}^{(\ell)}$. So, it follows from Corollary 2.6 that all $b_{j}$ belong to the center of $\mathscr{F}_{\ell, 1}^{\text {loc }}$. Consequently, $Z_{j} \stackrel{\text { def }}{=} b_{j}[\mathrm{~T}] \in \mathcal{Z}(\mathcal{W}(\mathrm{T}))$, by Theorem 3.8 Moreover, $b_{j}=b_{j}^{*} b_{j}$ (because both these functions coincide on the set of all irreducible $\ell$-tuples), and $b_{j} b_{j^{\prime}}=0$ for distinct $j$ and $j^{\prime}$, which implies that $Z_{j}$ are mutually orthogonal projections. Finally, since the partial sums of $\sum_{j \in J} b_{j}$ are uniformly bounded and converge pointwise to the unit $\mathfrak{j}$ of $\mathscr{F}_{\ell, 1}$, we conclude that the partial sums of $\sum_{j \in J} Z_{j}$ converge to the unit of $\mathcal{W}(\mathrm{T})$.

Now assume $b_{j}, b_{s}^{\prime}, u_{j}$ and $u_{s}^{\prime}$ are as specified in (B). We deduce from part (A) and Lemma 3.9 that both the operators $R \stackrel{\text { def }}{=} \sum_{j \in J} u_{j}[\mathrm{~T}] b_{j}[\mathrm{~T}]$ and $R^{\prime} \stackrel{\text { def }}{=}$ $\sum_{s \in S} u_{s}^{\prime}[\mathrm{T}] b_{s}^{\prime}[\mathrm{T}]$ are well defined and affiliated with $\mathcal{W}(\mathrm{T})$. To show that $R=R^{\prime}$, for any $\lambda \stackrel{\text { def }}{=}(j, s) \in \Lambda \stackrel{\text { def }}{=} J \times S$ we put $v_{\lambda} \stackrel{\text { def }}{=} b_{j} b_{s}^{\prime}$. Observe that $u_{j} v_{(j, s)}=u_{s}^{\prime} v_{(j, s)}$ (by (3-5) ), $u_{j} b_{j}=\sum_{s \in S} u_{j} v_{(j, s)}$ and similarly $u_{s}^{\prime} b_{s}^{\prime}=\sum_{j \in J} u_{s}^{\prime} v_{(j, s)}$ (and partial sums of both these series are uniformly bounded), from which we deduce that:

- $u_{j}[\mathbf{T}] v_{(j, s)}[\mathbf{T}]=u_{s}^{\prime}[\mathbf{T}] v_{(j, s)}[\mathbf{T}] ;$ and

- $u_{j}[\mathbf{T}] b_{j}[\mathbf{T}]$ is the limit of $\sum_{s \in S} u_{j}[\mathrm{~T}] v_{(j, s)}[\mathrm{T}]$ in the $*$-strong operator topology; and

- $u_{s}^{\prime}[\mathrm{T}] b_{s}^{\prime}[\mathrm{T}]$ is the limit of $\sum_{j \in J} u_{s}^{\prime}[\mathrm{T}] v_{(j, s)}[\mathrm{T}]$ in the $*$-strong operator topology.

Since the summands of each of the series that appear above have mutually orthogonal disjoint ranges, we conclude that for any $x \in \mathcal{H}$,

$$
\sum_{j \in J}\left\|u_{j}[\mathrm{~T}] b_{j}[\mathrm{~T}] x\right\|^{2}=\sum_{j \in J} \sum_{s \in S}\left\|u_{j}[\mathrm{~T}] v_{(j, s)}[\mathrm{T}] x\right\|^{2}=\sum_{s \in S}\left\|u_{s}^{\prime}[\mathrm{T}] b_{s}^{\prime}[\mathrm{T}] x\right\|^{2},
$$

which shows that the domains of $R$ and $R^{\prime}$ coincide. An analogous argument proves that for any $x$ in this common domain, $\sum_{j \in J} u_{j}[\mathbf{T}] b_{j}[\mathrm{~T}] x=\sum_{s \in S} u_{s}^{\prime}[\mathbf{T}] b_{s}^{\prime}[\mathbf{T}] x$ and we are done.

3.11. Proposition. Let $\mathrm{T}$ be an $\mathrm{FTI} \ell$-tuple of bounded operators on a Hilbert space $\mathcal{H}$. There exists a unique unital $*$-homomorphism $\mathscr{F}_{\ell, 1} \ni f \mapsto f[\mathrm{~T}] \in \hat{\mathcal{W}}$ such that (F1), (F3) and (F5) hold where $\mathcal{W} \stackrel{\text { def }}{=} \mathcal{W}(\mathrm{T})$. Moreover, if $\mathcal{H}$ is separable, then

$$
\begin{aligned}
\left\{u[\mathrm{~T}]: u \in \mathscr{F}_{\ell, 1}\right\} & =\hat{\mathcal{W}}, \\
\left\{u[\mathrm{~T}]: u \in \mathcal{Z}\left(\mathscr{F}_{\ell, 1}\right)\right\} & =\mathcal{Z}(\hat{\mathcal{W}}) .
\end{aligned}
$$

Proof. The uniqueness part, as usual, follows from Theorem 2.1 and is left to the reader. Here we focus only on the existence part. Let $\Phi: \mathscr{F}_{\ell, 1}^{\text {loc }} \ni u \mapsto u[T] \in \mathcal{W}$ be a unital $*$-homomorphism guaranteed by Theorem 3.8. Fix an arbitrary $u \in \mathscr{F}_{\ell, 1}$. For any $n>0$, let $\mathscr{B}_{n}$ consist of all irreducible $\ell$-tuples $\mathrm{X} \in \mathscr{M}^{(\ell)}$ such that $n-1 \leqslant\|u(\mathrm{X})\|<n$. Observe that $U . \mathrm{X} \in \mathscr{B}_{n}$ for each $\mathrm{X} \in \mathscr{B}_{n}$ and $U \in \mathscr{U}_{d(\mathrm{X})}$. We therefore conclude (using Corollary 2.5) that there exists $b_{n} \in \mathscr{F}_{\ell, 1}^{b d}$ which vanishes at each irreducible $\ell$-tuple from $\mathscr{M}^{(\ell)} \backslash \mathscr{B}_{n}$ and satisfies $b_{n}(\mathrm{X})=I_{d(\mathrm{X})}$ for any $\mathbf{X} \in \mathscr{B}_{n}$. Then $u b_{n} \in \mathscr{F}_{\ell, 1}^{b d}$ for all $n, b_{n}$ are mutually disjoint and $u$ is the pointwise limit of the series $\sum_{n=1}^{\infty}\left(u b_{n}\right) b_{n}$. We define $u[T]$ as $\sum_{n=1}^{\infty} \Phi\left(u b_{n}\right) \Phi\left(b_{n}\right)$. 
It follows from Lemma 3.10 that $u[T] \in \hat{\mathcal{W}}$. A standard argument proves that the aforementioned operator $u[\mathrm{~T}]$ coincides with $\Phi(u)$ for $u \in \mathscr{F}_{\ell, 1}^{l o c}$. Consequently, conditions (F1), (F3) and (F5) are fulfilled. To check that the assignment $u \mapsto u[\mathrm{~T}]$ defines a $*$-homomorphism from $\mathscr{F}_{\ell, 1}$ into $\hat{\mathcal{W}}$, fix $u$ and $v$ in $\mathscr{F}_{\ell, 1}$. We argue similarly as before. Let $\mathscr{B}_{n}^{\prime}$ constist of all irreducible $\mathrm{X} \in \mathscr{M}^{(\ell)}$ for which $n-$ $1 \leqslant \max (\|u(\mathbf{X})\|,\|v(\mathbf{X})\|)<n$ and $b_{n}^{\prime} \in \mathscr{F}_{\ell, 1}^{b d}$ be a function that vanishes at each irreducible $\ell$-tuple from $\mathscr{M}^{(\ell)} \backslash \mathscr{B}_{n}^{\prime}$ and satisfies $b_{n}^{\prime}(\mathrm{X})=I_{d(\mathrm{X})}$ for any $\mathrm{X} \in \mathscr{B}_{n}^{\prime}$. Then the functions $u b_{n}^{\prime}$ and $v b_{n}^{\prime}$ are bounded and thus Lemma 3.10 implies that $u[\mathrm{~T}]=$ $\sum_{n=1}^{\infty} \Phi\left(u b_{n}^{\prime}\right) \Phi\left(b_{n}^{\prime}\right)$ and $v[\mathrm{~T}]=\sum_{n=1}^{\infty} \Phi\left(v b_{n}^{\prime}\right) \Phi\left(b_{n}^{\prime}\right)$. A similar reasoning shows that also $(u v)[\mathrm{T}]=\sum_{n=1}^{\infty} \Phi\left(u v b_{n}^{\prime}\right) \Phi\left(b_{n}^{\prime}\right)$ and $(\alpha u+\beta v)[\mathrm{T}]=\sum_{n=1}^{\infty} \Phi\left((\alpha u+\beta v) b_{n}^{\prime}\right) \Phi\left(b_{n}^{\prime}\right)$ for all scalars $\alpha, \beta \in \mathbb{C}$. Now it is enough to apply Lemma 3.9.

We turn to the additional claim of the proposition. Assume $\mathcal{H}$ is separable. Since for the centra the proof goes similarly (because $\mathcal{Z}(\hat{\mathcal{W}})=\hat{\mathcal{Z}}$ where $\mathcal{Z}=\mathcal{Z}(\mathcal{W})$, see [40]), we shall show only the first additional conclusion. To this end, we fix $S \in \hat{\mathcal{W}}$. It was shown in 40 ] that then there exist $A \in \mathcal{W}$ and a sequence $Z_{1}, Z_{2}, \ldots$ of mutually orthogonal projections in $\mathcal{Z}(\mathcal{W})$ that sum up to the unit of $\mathcal{W}$ and satisfy $S=\sum_{n=1}^{\infty} n A Z_{n}$. Further, let $\lambda$ be a probabilistic measure as specified in Proposition 3.6. We conclude from that result that there are functions $v \in \mathscr{F}_{\ell, 1}^{b d}$ and $b_{n} \in \mathcal{Z}\left(\mathscr{F}_{\ell, 1}^{b d}\right)$ such that $v[\mathrm{~T}]=A, b_{n}[\mathrm{~T}]=Z_{n}$ and $\left\|b_{n}\right\| \leqslant 1$. Further, since $b_{n}[\mathbf{T}] b_{m}[\mathbf{T}]=0$ for distinct $n$ and $m$ and $b_{n}^{2}[\mathrm{~T}]=b_{n}[\mathrm{~T}]$, property $\left(\Lambda 0^{\prime}\right)$ yields that $b_{n} b_{m}=0$ and $b_{n}^{2}=b_{n} \lambda$-almost everywhere. Let $\mathscr{A} \in \mathfrak{B}\left(\mathscr{M}^{(\ell)}\right)$ be a set of full $\lambda$-measure on which all the aforementioned equations hold. Then $b_{n}(\mathbf{X}) \in$ $\left\{0, I_{d(\mathrm{X})}\right\}$ for any irreducible $X \in \mathscr{A}$. We leave it as an exercise that we may modify the sequence $b_{1}, b_{2}, \ldots$ to obtain mutually disjoint functions $b_{1}^{\prime}, b_{2}^{\prime}, \ldots \in \mathscr{F}_{\ell, 1}^{b d}$ such that $b_{n}^{\prime}$ and $b_{n}$ are equal $\lambda$-almost everywhere and $\sum_{n=1}^{\infty} b_{n}(\mathrm{X})=I_{d(\mathrm{X})}$ for each irreducible $\ell$-tuple $\mathrm{X} \in \mathscr{M}^{(\ell)}$. Then $b_{n}^{\prime}[\mathrm{T}]=b_{n}[\mathrm{~T}]=Z_{n}$, the pointwise limit $u$ of $\sum_{n=1}^{\infty} n v b_{n}^{\prime}$ belongs to $\mathscr{F}_{\ell, 1}$ and Lemma 3.10 yields that $u[\mathrm{~T}]=\sum_{n=1}^{\infty} n v[\mathrm{~T}] b_{n}^{\prime}[\mathrm{T}]=$ $S$, which finishes the proof.

Proof of Theorem 3.1. As usual, uniqueness follows from Theorem 2.1. First we shall show existence and after that we shall establish properties (F6)-(F9). To avoid misundestandings, the functional calculus $u \mapsto u[\mathrm{~T}]$ for $\ell$-tuples $\mathrm{T}$ of bounded operators obtained in Proposition 3.11 shall be denoted by $u \mapsto \Phi_{\mathrm{\top}}(u)$. It follows from the results of [40] that there is a sequence $\mathcal{H}_{1}, \mathcal{H}_{2}, \ldots$ of mutually orthogonal reducing subspaces for $\mathrm{T}$ such that $\mathcal{H}=\bigoplus_{n=1}^{\infty} \mathcal{H}_{n}$ and $\left.\mathrm{T}^{(n)} \stackrel{\text { def }}{=} \mathrm{T}\right|_{\mathcal{H}_{n}}$ consists of bounded operators. For any $u \in \mathscr{F}_{\ell, 1}$ we define $u[\mathrm{~T}]$ as $\bigoplus_{n=1}^{\infty} \Phi_{\mathrm{T}^{(n)}}(u)$. Since $\mathscr{F}_{\ell, 1}^{b d}$ is a $C^{*}$-algebra (and the functions $\Phi_{\mathrm{T}^{(n)}}$ are $*$-homomorphisms), we infer that $\left\|\Phi_{\mathrm{T}^{(n)}}(u)\right\| \leqslant\|u\|$ for any $u \in \mathscr{F}_{\ell, 1}^{b d}$. This shows (F3), from which one deduces (F5); whereas (F1) and (F4) are straightforward. Finally, defining, for any $u \in \mathscr{F}_{\ell, \ell^{\prime}}$, $u[\mathrm{~T}]$ as $\left(\left(\pi_{1}^{\left(\ell^{\prime}\right)} \circ u\right)[\mathrm{T}], \ldots,\left(\pi_{\ell^{\prime}}^{\left(\ell^{\prime}\right)} \circ u\right)[\mathrm{T}]\right)$, we see that $(\mathrm{F} 2)$ holds.

Further, (F7) is covered by Theorem 3.8, whereas (F8) follows from Theorem 2.1 (and (F5)). Also (F6) follows from Theorem 2.1. Indeed, it suffices to check (F6) for $\ell^{\prime \prime}=1$. To this end, we fix $u \in \mathscr{F}_{\ell, \ell^{\prime}}$ and put $\mathcal{E} \stackrel{\text { def }}{=}\left\{v \in \mathscr{F}_{\ell, 1}:(v \circ u)[\mathrm{T}]=v[u[\mathrm{~T}]]\right\}$. We infer from (F2) and (F4)-(F5) that conditions (E0)-(E2) are satisfied. Thus, $\mathcal{E}=\mathscr{F}_{\ell, 1}$ and we are done.

We turn to (F9). Let $\mathfrak{u} \in \mathscr{F}_{\ell, \ell}$ be any function such that

$$
\mathfrak{u}\left(X_{1}, \ldots, X_{\ell}\right)=\left(X_{1}\left(I_{d\left(X_{1}\right)}-\left|X_{1}\right|\right)^{-1}, \ldots, X_{\ell}\left(I_{d\left(X_{\ell}\right)}-\left|X_{\ell}\right|\right)^{-1}\right)
$$

for any $\left(X_{1}, \ldots, X_{\ell}\right) \in \mathscr{M}^{(\ell)}$ with $\left\|X_{j}\right\|<1$ for each $j$. Put $\mathrm{S} \stackrel{\text { def }}{=} \mathfrak{b}(\mathrm{T})$. Then $\mathcal{W}=\mathcal{W}(S)$. Below we shall think of the $\mathfrak{b}$-transform as of a function in $\mathscr{F}_{\ell, \ell}$. 
Straightforward calculations show that $\mathfrak{u}(\mathfrak{b}(\mathrm{X}))=\mathrm{X}$ for any $\mathrm{X} \in \mathscr{M}^{(\ell)}$. It is easy to verify that $\mathfrak{b}[T]=S$ (use the uniqueness of the square root of a nonnegative selfadjoint unbounded operator). Since $S$ consists of bounded operators, Propositions 3.6 and 3.11 yield that

$$
\begin{aligned}
\left\{f[\mathrm{~S}]: \quad f \in \mathscr{F}_{\ell, 1}^{b d}\right\} & =\mathcal{W}, \\
\left\{f[\mathrm{~S}]: f \in \mathcal{Z}\left(\mathscr{F}_{\ell, 1}^{b d}\right)\right\} & =\mathcal{Z}(\mathcal{W}), \\
\left\{f[\mathrm{~S}]: \quad f \in \mathscr{F}_{\ell, 1}\right\} & =\hat{\mathcal{W}}, \\
\left\{f[\mathrm{~S}]: \quad f \in \mathcal{Z}\left(\mathscr{F}_{\ell, 1}\right)\right\} & =\mathcal{Z}(\hat{\mathcal{W}}) .
\end{aligned}
$$

But (F6) implies that for any $f \in \mathscr{F}_{\ell, 1}, f[\mathrm{~T}]=(f \circ \mathfrak{u})[\mathrm{S}]$ and thus (F9) follows from the above formulas.

Proof of Theorem 1.1. We leave it to the reader that all conclusions of the theorem follow from the results of this section.

Also the proof of the following result is skipped.

3.12. Corollary. Let $f \in \mathscr{F}_{\ell, \ell^{\prime}}$.

(A) If $\mathrm{T}$ is an $\mathrm{FTI} \ell$-tuple of operators in $\mathcal{H}$ and $U: \mathcal{H} \rightarrow \mathcal{K}$ is a unitary operator, then $f[U . T]=U . f[\mathrm{~T}]$.

(B) If $\left\{\mathrm{T}^{(s)}\right\}_{s \in S}$ is an arbitrary collection of $\mathrm{FTI} \ell$-tuples, then $f\left[\bigoplus_{s \in S} \mathrm{~T}^{(s)}\right]=$ $\bigoplus_{s \in S} f\left[\mathrm{~T}^{(s)}\right]$.

(C) For any $\mathrm{X} \in \mathscr{M}^{(\ell)}, f[\mathrm{X}]=f(\mathrm{X})$.

\section{SPECTRAL THEOREM AND SPECTRUM}

In this section we apply the functional calculus built in the previous section to propose a new approach to so-called operator-valued spectra and some variations of the spectral theorem.

We begin with

4.1. Proposition. Let $\mathrm{T}$ be an $\mathrm{FTI} \ell$-tuple of operators. For each $n>0$, denote by $j_{n}$ the function in $\mathscr{F}_{\ell, 1}^{b d}$ which vanishes at each irreducible $\ell$-tuple in $\mathscr{M}^{(\ell)} \backslash \mathscr{M}_{n}^{\ell}$ and sends each irreducible $\ell$-tuple in $\mathscr{M}_{n}^{\ell}$ to $I_{n}$. Then $j_{n}[\mathrm{~T}]$ is the greatest selfadjoint projection $Z \in \mathcal{Z}\left(\mathcal{W}^{\prime \prime}(\mathrm{T})\right)$ such that $Z \mathcal{W}^{\prime \prime}(\mathrm{T})=\left\{Z A: A \in \mathcal{W}^{\prime \prime}(\mathrm{T})\right\}$ is type $\mathrm{I}_{n}$.

Proof. Since $\mathcal{W} \stackrel{\text { def }}{=} \mathcal{W}^{\prime \prime}(\mathrm{T})$ is finite and type $\mathrm{I}$, there is a sequence $Z_{1}, Z_{2}, \ldots$ of mutually orthogonal projections in $\mathcal{Z}(\mathcal{W})$ that sum up to the unit of $\mathcal{W}$ and are such that $Z_{n} \mathcal{W}$ is type $\mathrm{I}_{n}$ (or $Z_{n}=0$ ). Our task is to show that $j_{n}[\mathrm{~T}]=Z_{n}$. Denoting by $\mathrm{T}^{(n)}$ the restriction of $\mathrm{T}$ to the range of $Z_{n}$, we obtain that $\mathrm{T}=$ $\bigoplus_{n=1}^{\infty} \mathrm{T}^{(n)}$ and $\mathcal{W}^{\prime \prime}\left(\mathrm{T}^{(n)}\right)$ is type $\mathrm{I}_{n}$. Since $u[\mathrm{~T}]=\bigoplus_{n=1}^{\infty} u\left[\mathrm{~T}^{(n)}\right]$ for any $u \in \mathscr{F}_{\ell, 1}$ (by Corollary 3.12), we see that it suffices to check that $j_{n}\left[\mathrm{~T}^{(n)}\right]$ coincides with the unit of $\mathcal{W}^{\prime \prime}\left(\mathrm{T}^{(n)}\right)$ (that is, with $Z_{n}$ ). This reduces the issue to the case when $\mathcal{W}$ is type $\mathrm{I}_{N}$ (and we only need to verify that $j_{N}[\mathrm{~T}]$ is the unit of $\mathcal{W}$ ). As in the proof of Theorem 3.1, we conclude from the results of [40] that $\mathrm{T}=\bigoplus_{s \in S} \mathrm{~T}^{(s)}$ where each of $\mathrm{T}^{(s)}$ consists of bounded operators acting in a separable Hilbert space (and $\mathcal{W}^{\prime \prime}\left(\mathrm{T}^{(s)}\right)$ is type $\left.\mathrm{I}_{N}\right)$. Now condition $(\Lambda 0)$ of Lemma 3.5 shows that $j_{N}\left[\mathrm{~T}^{(s)}\right]=\mathfrak{j}\left[\mathrm{T}^{(s)}\right]$ for any $s \in S$ (recall that $\mathfrak{j}$ is the unit of $\mathscr{F}_{\ell, 1}$ ). Thus, again thanks to Corollary $3.12, j_{N}[\mathrm{~T}]=\mathfrak{j}[\mathrm{T}]$ and we are done.

4.2. Lemma. Let $\mathrm{T}$ be an $\mathrm{FTI} \ell$-tuple and $f$ be any function in $\mathscr{F}_{\ell, \ell^{\prime}}$. Let $u \in \mathscr{F}_{\ell, 1}^{b d}$ be a (unique) function such that for any irreducible $\ell$-tuple $\mathrm{X} \in \mathscr{M}^{(\ell)}, u(\mathrm{X})$ is 
the orthogonal projection onto the range of $\sum_{j=1}^{\ell^{\prime}}\left(\pi_{j}^{\left(\ell^{\prime}\right)}(f(\mathrm{X}))\right)^{*}\left(\pi_{j}^{\left(\ell^{\prime}\right)}(f(\mathrm{X}))\right)$. Then $f[\mathrm{~T}]=(0, \ldots, 0)$ iff $u[\mathrm{~T}]=0$.

Proof. Let $g \stackrel{\text { def }}{=} \sum_{j=1}^{\ell^{\prime}}\left(\pi_{j}^{\left(\ell^{\prime}\right)} \circ f\right)^{*} \cdot\left(\pi_{j}^{\left(\ell^{\prime}\right)} \circ f\right)$. Since $0 \leqslant\left(\pi_{j}^{\left(\ell^{\prime}\right)} \circ f\right)^{*}\left(\pi_{j}^{\left(\ell^{\prime}\right)} \circ f\right) \leqslant g$, it follows that $0 \leqslant\left(\pi_{j}^{\left(\ell^{\prime}\right)}[f[\mathrm{~T}]]\right)^{*} \cdot\left(\pi_{j}^{\left(\ell^{\prime}\right)}[f[\mathrm{~T}]]\right) \leqslant g[\mathrm{~T}]$ and, consequently, $f[\mathrm{~T}]=0$ if and only if $g[\mathbf{T}]=0$. So, we only need to show that $g[\mathrm{~T}]=0$ iff $u[\mathrm{~T}]=0$. To this end, observe that for any $\mathrm{X} \in \mathscr{M}^{(\ell)}$ there exists a positive integer $N>0$ for which $2^{-N} g(\mathrm{X}) \leqslant u(\mathrm{X}) \leqslant 2^{N} g(\mathrm{X})$. One concludes that therefore there is a sequence $b_{1}, b_{2}, \ldots \in \mathscr{F}_{\ell, 1}^{b d}$ of mutually disjoint functions that sum up to the unit matrix at each irreducible $\ell$-tuple and satisfy $2^{-n} g b_{n} \leqslant u b_{n} \leqslant 2^{n} g b_{n}$ (globally) for any $n$. So, the assertion readily follows from the fact that $u[\mathrm{~T}]=\sum_{n=1}^{\infty}\left(u b_{n}\right)[\mathrm{T}] b_{n}[\mathrm{~T}]$ and similarly $g[\mathrm{~T}]=\sum_{n=1}^{\infty}\left(g b_{n}\right)[\mathrm{T}] b_{n}[\mathrm{~T}]$.

We would like to think of $u[\mathrm{~T}]$ as of the integral $\int_{\mathscr{M}^{(\ell)}} u \mathrm{~d} E$ with respect to some spectral measure $E$ (this shall be explained in more detail in the sequel). Under such a thinking, it is a typical question of when $u[\mathrm{~T}]=0$ for a nonnegative function $u \in \mathscr{F}_{\ell, 1}$. The foregoing result reduces the above problem to functions that are selfadjoint projections (as members of $C^{*}$-algebras).

Lemma 4.2 is quite simple and intuitive. The following result is much more subtle.

4.3. Proposition. For a function $f \in \mathscr{F}_{\ell, \ell^{\prime}}$, let $v \in \mathcal{Z}\left(\mathscr{F}_{\ell, 1}^{b d}\right)$ be a (unique) function such that for any irreducible $\ell$-tuple $\mathrm{X} \in \mathscr{M}^{(\ell)}, v(\mathrm{X})=0$ if $f(\mathrm{X})=(0, \ldots, 0)$ and $v(\mathrm{X})=I_{d(\mathrm{X})}$ otherwise. Then, for any $\mathrm{FTI} \ell$-tuple $\mathrm{T}, f[\mathrm{~T}]=(0, \ldots, 0)$ iff $v[\mathrm{~T}]=0$.

Proof. Let $u \in \mathscr{F}_{\ell, 1}^{b d}$ be as specified in Lemma 4.2 from which we infer that $f[\mathrm{~T}]=0$ iff $u[\mathrm{~T}]=0$. Observe that for any irreducible $\mathrm{X} \in \mathscr{M}^{(\ell)}, u(\mathrm{~T})=0$ precisely when $v(\mathrm{X})=0$. One concludes that therefore

$$
u(\mathrm{X})=0 \Longleftrightarrow v(\mathrm{X})=0 \quad\left(\mathrm{X} \in \mathscr{M}^{(\ell)}\right) .
$$

Further, it follows from the results of $\left[40\right.$, that $\mathrm{T}=\bigoplus_{n=1}^{\infty} \mathrm{T}^{(n)}$ where each $\mathrm{T}^{(n)}$ consists of bounded operators (cf. the proof of Theorem 3.1). So, Corollary 3.12 implies that $u[\mathrm{~T}]=0$ iff $u\left[\mathrm{~T}^{(n)}\right]=0$ for all $n$ (and analogously for $v$ in place of $u$ ). This argument reduces the issue to the case when $T$ is bounded. Then $\mathrm{T}=\bigoplus_{s \in S} \mathrm{~T}^{(s)}$ where each $\mathrm{T}^{(s)}$ acts on a separable Hilbert space. So, arguing as before, we see that it suffices to prove the proposition for $\ell$-tuples $T$ of bounded operators acting on a separable Hilbert space. In that case we apply Proposition 3.6. Let a measure $\lambda$ be as specified there. It then follows from $\left(\Lambda 0^{\prime}\right)$ that $u[T]=0$ if and only if $u=0 \lambda$-almost everywhere (and the same for $v$ in place of $u$ ). So, a look at (4-1) finishes the proof.

Noticing that selfadjoint projections in $\mathcal{Z}\left(\mathscr{F}_{\ell, 1}^{b d}\right)$ correspond to unitarily invariant Borel sets of irreducible $\ell$-tuples of matrices, based on Proposition 4.3, we introduce

4.4. Definition. Let $\mathfrak{B}^{(\ell)}$ be the family of all Borel sets $\mathscr{B}$ of irreducible $\ell$-tuples of matrices such that $U . \mathrm{X} \in \mathscr{B}$ whenever $\mathrm{X} \in \mathscr{B}$ and $U \in \mathscr{U}_{d(\mathrm{X})}$ (every such a set is called unitarily invariant). $\mathfrak{B}^{(\ell)}$ is a $\sigma$-algebra of subsets of the set $\mathscr{I}^{(\ell)}$ of all irreducible $\ell$-tuples in $\mathscr{M}^{(\ell)}$. For any set $\mathscr{B} \in \mathfrak{B}^{(\ell)}$ there exists a unique function in $\mathscr{F}_{\ell, 1}$, to be denoted by $\mathfrak{j}_{\mathscr{B}}$, that vanishes at each irreducible $\ell$-tuple in $\mathscr{I}^{(\ell)} \backslash \mathscr{B}$ and sends each member of $\mathscr{B}$ to the unit matrix of a respective degree. For simplicity, we shall use $\mathscr{I}_{n}^{(\ell)}$ to denote the set $\mathscr{I}^{(\ell)} \cap \mathscr{M}_{n}^{\ell}$.

For any FTI $\ell$-tuple $\mathrm{T}$ of operators, the spectral measure of $\mathrm{T}$ is the set function $E_{\mathrm{T}}: \mathfrak{B}^{(\ell)} \rightarrow \mathcal{Z}(W w W(\mathrm{~T}))$ given by $E_{\mathrm{T}}(\mathscr{B}) \stackrel{\text { def }}{=} \mathfrak{j}_{\mathscr{B}}[\mathrm{T}]$ (that $E_{\mathrm{T}}(\mathscr{B})$ belongs to $\mathcal{Z}(\mathcal{W}(\mathrm{T}))$ follows from property (F3) in Theorem 3.1). 
We recall that an operator-valued set function $E: \mathfrak{M} \rightarrow \mathcal{B}(\mathcal{H})$ (where $\mathfrak{M}$ is a $\sigma$-algebra on a set $X$ and $\mathcal{H}$ is a Hilbert space) is said to be a spectral measure if

- $E(X)$ is the identity operator on $\mathcal{H}$; and

- $E(\sigma)$ is an orthogonal projection for any $\sigma \in \mathfrak{M}$; and

- $E\left(\sigma \cap \sigma^{\prime}\right)=E(\sigma) E\left(\sigma^{\prime}\right)$ for all $\sigma, \sigma^{\prime} \in \mathfrak{M}$; and

- whenever $\sigma_{1}, \sigma_{2}, \ldots$ are pairwise disjoint sets in $\mathfrak{M}$, the series $\sum_{n=1}^{\infty} E\left(\sigma_{n}\right)$ converges to $E\left(\bigcup_{n=1}^{\infty} \sigma_{n}\right)$ in the strong operator topology.

The proofs of the next two result are left to the reader.

4.5. Lemma. $\mathscr{I}^{(\ell)}$ is a locally compact Polish space and for any $\mathrm{FTI} \ell$-tuple $\mathrm{T}, E_{\mathrm{T}}$ is (indeed) a spectral measure. In particular, there exists the smallest set, denoted by $\operatorname{supp}\left(E_{\mathrm{T}}\right)$, among all sets $\mathscr{B} \in \mathfrak{B}^{(\ell)}$ that are relatively closed in $\mathscr{I}^{(\ell)}$ and satisfy $E_{\mathrm{T}}\left(\mathscr{I}^{(\ell)} \backslash \mathscr{B}\right)=0$.

4.6. Corollary. Let $\mathrm{T}$ be an $\mathrm{FTI} \ell$-tuple. For any two functions $u$ and $v$ in $\mathscr{F}_{\ell, \ell^{\prime}}$, the set $D(u, v) \stackrel{\text { def }}{=}\left\{\mathrm{X} \in \mathscr{I}^{(\ell)}: u(\mathrm{X}) \neq v(\mathbf{X})\right\}$ belongs to $\mathfrak{B}^{(\ell)}$, and

$$
u[\mathrm{~T}]=v[\mathrm{~T}] \Longleftrightarrow E_{\mathrm{T}}(D(u, v))=0 .
$$

4.7. Corollary. Let $\mathrm{T}$ be an $\mathrm{FTI} \ell$-tuple of operators in $\mathcal{H}$ and $u \in \mathscr{F}_{\ell, 1}$. If $u[\mathrm{~T}]$ is one-to-one, then the range of $u[\mathrm{~T}]$ is dense in $\mathcal{H}$ and there is $v \in \mathscr{F} \ell, 1$ for which $v[\mathrm{~T}]=(u[\mathrm{~T}])^{-1}$.

Proof. For simplicity, we put $S \stackrel{\text { def }}{=} u[\mathrm{~T}]$ and $\mathcal{W} \stackrel{\text { def }}{=} \mathcal{W}^{\prime \prime}(S)$. Let $S=Q|S|$ (where $|S|=\left(S^{*} S\right)^{1 / 2}$ ) be the polar decomposition of $S$ (so, $Q$ is a partial isometry whose kernel coincides with the kernel of $S$ ). Since $S \in \hat{\mathcal{W}}$, we conclude that $Q \in \mathcal{W}$. So, $Q$ is a unitary operator, being an isometry in a finite von Neumann algebra $\mathcal{W}$. Since $|S|$ is selfadjoint and one-to-one, the range of $|S|$ is dense in $\mathcal{H}$. Thus, so is the range of $S$. It is now an easy observation that $S^{-1}$ is affiliated with $\mathcal{W}$. We shall prove that $S^{-1}$ is the value of the functional calculus for T.

Put $\mathscr{Z} \stackrel{\text { def }}{=}\left\{\mathrm{X} \in \mathscr{I}^{(\ell)}: \operatorname{det}(u(\mathrm{X}))=0\right\}$. Observe that $\mathscr{Z} \in \mathfrak{B}^{(\ell)}$. We claim that $E_{\mathrm{T}}(\mathscr{Z})=0$. Suppose, on the contrary, that $E_{\mathrm{T}}(\mathscr{Z})$ is nonzero. Then there in $N>0$ such that

$$
E_{\mathrm{T}}\left(\mathscr{Z} \cap \mathscr{M}_{N}^{\ell}\right) \neq 0 .
$$

Consider a multifunction $\Psi$ on $\mathscr{D} \stackrel{\text { def }}{=} \mathscr{I}^{(\ell)} \cap \mathscr{M}_{N}^{\ell}$ which assigns to each $\ell$-tuple $\mathrm{X} \in \mathscr{D}$ the kernel of the matrix $u(\mathbf{X})$. So, $\Psi(\mathbf{X}) \neq\{0\}$ if and only if $\mathbf{X} \in \mathscr{Z}$. Equipping the set of all linear subspaces of $\mathbb{C}^{N}$ with the Effros-Borel structure (see 13, 14 or $\S 6$ in Chapter V in 50 and Appendix there), we conclude that $\Psi$ is measurable (this is a kind of folklore; it may also be simply deduced e.g. from a combination of Proposition 2.4 in [15] and Corollary A.18 in [50]). So, it follows from Effros' theory that there exist measurable functions $h_{1}, h_{2}, \ldots: \mathscr{D} \rightarrow \mathbb{C}^{N}$ such that the set $\left\{h_{n}(\mathrm{X}): n>0\right\}$ is a dense subset of $\Psi(\mathrm{X})$ for each $\mathrm{X} \in \mathscr{D}$ (to convince oneself of that, consult e.g. subsection A.16 of Appendix in [51]). We shall now modify $h_{n}$ to obtain unitarily invariant functions with analogous properties. To this end, we fix a Borel kernel $\mathscr{K}$ of $\mathscr{M}^{(\ell)}$. Let $\tau: \mathscr{D} \rightarrow \mathscr{K} \cap \mathscr{M}_{N}^{\ell}$ be the function that assigns to each $\mathrm{X} \in \mathscr{D}$ the unique point of $\left\{U . \mathrm{X}: U \in \mathscr{U}_{N}\right\} \cap \mathscr{K}$. We leave the proof that $\tau$ is Borel as an exercise. Now let $g_{n} \stackrel{\text { def }}{=} h_{n} \circ \tau$. We see that $g_{n}(U . \mathrm{X})=g_{n}(\mathrm{X})$ for any $\mathrm{X} \in \mathscr{D}$ and $U \in \mathscr{U}_{N}$. What is more, $\left\{g_{n}(\mathrm{X}): n>0\right\}=\left\{h_{n}(\mathrm{X}): n>0\right\}$ for $\mathrm{X} \in \mathscr{D} \cap \mathscr{K}$. So, since $\Psi(U . \mathrm{X})=\Psi(\mathrm{X})$ for all $\mathrm{X} \in \mathscr{D}$ and $U \in \mathscr{U}_{N}$, we conclude that

(d) $\left\{g_{n}(\mathrm{X}): n>0\right\}$ is a dense subset of $\Psi(\mathrm{X})$ for each $\mathrm{X} \in \mathscr{D}$.

But now, the sets $\left\{\mathrm{X} \in \mathscr{D}: g_{n}(\mathrm{X}) \neq 0\right\}$ belong to $\mathfrak{B}^{(\ell)}$. Hence, it follows from (4-2) and (d) that there is $g \in\left\{g_{n}: n>0\right\}$ with

$$
E_{\mathrm{T}}\left(\mathscr{Z}_{g}\right) \neq 0
$$


where $\mathscr{Z}_{g} \stackrel{\text { def }}{=}\{\mathrm{X} \in \mathscr{D}: g(\mathrm{X}) \neq 0\}$. Let $w_{0}: \mathscr{K} \rightarrow \mathscr{M}$ be defined as follows: if $\mathrm{X} \in \mathscr{K} \cap \mathscr{Z}_{g}, w_{0}(\mathrm{X})$ is the matrix which corresponds (in the canonical basis of $\mathbb{C}^{N}$ ) to a linear operator

$$
\mathbb{C}^{N} \ni \xi \mapsto \frac{\langle\xi, g(\mathrm{X})\rangle}{\langle g(\mathrm{X}), g(\mathrm{X})\rangle} g(\mathrm{X}) \in \mathbb{C}^{N}
$$

(where $\langle\cdot,-\rangle$ denotes the standard inner product in $\mathbb{C}^{N}$ ), and $w_{0}(\mathbf{X})=0 \in \mathscr{M}_{d(\mathrm{X})}$ for any $\mathrm{X} \in \mathscr{K} \backslash \mathscr{Z}_{g}$. Note that $u w_{0}$ vanishes at each point of $\mathscr{K}$. Finally, let $w \in \mathscr{F}_{\ell, 1}$ be a unique extension of $w_{0}$, guaranteed by Corollary 2.5. Then $u w$ is the zero function in $\mathscr{F}_{\ell, 1}$. So, $u[\mathrm{~T}] w[\mathrm{~T}]=0$. Since $u[\mathrm{~T}]$ is one-to-one, we see that $w[\mathrm{~T}]=$. But $\mathscr{Z}_{g} \subset\left\{\mathbf{X} \in \mathscr{M}^{(\ell)}: w(\mathbf{X}) \neq 0\right\}$ and therefore $w[\mathrm{~T}]$ is nonzero, by (4-3) and Corollary 4.6. This contradiction shows that indeed $E_{\mathrm{T}}(\mathscr{Z})=0$.

Now let $v$ be a unique function in $\mathscr{F}_{\ell, 1}$ such that $v(\mathbf{X})=(u(\mathbf{X}))^{-1}$ for $\mathbf{X} \in$ $\mathscr{I}^{(\ell)} \backslash \mathscr{Z}$ and $v(\mathrm{X})$ vanishes at each point of $\mathscr{Z}$. We then have $E_{\mathrm{T}}(D(u v, \mathfrak{j}))=$ $E_{\mathrm{T}}(D(v u, \mathfrak{j}))=0$. Consequently, Corollary 4.6 implies that both $u[\mathrm{~T}] \cdot v[\mathrm{~T}]$ and $v[\mathrm{~T}] \cdot u[\mathrm{~T}]$ coincide with the identity operator on $\mathcal{H}$. This means that $v[\mathrm{~T}]$ is the inverse of $u[\mathrm{~T}]$ as an element of the unital ring $\mathscr{F}_{\ell, 1}$. But so is $S^{-1}$ and therefore $v[\mathrm{~T}]=S^{-1}$.

4.8. Definition. For any $\mathrm{FTI} \ell$-tuple $\mathrm{T}$, the $\operatorname{set} \operatorname{supp}\left(E_{\mathrm{T}}\right)$ is called the principal spectrum of $\mathrm{T}$.

Operator-valued spectra of arbitrary bounded Hilbert space operators were studied by Ernest [15], Hadwin [21, 22] and others (see also, e.g., [29] and [32]).

Our next aim is to show that the functional calculus for FTI tuples, as for normal operators, may be obtained as an effect of integration (with respect to certain operator-valued measures). It is worth noticing here that the way we shall do this will be ambiguous, that is, it will depend on a kernel (of $\mathscr{M}^{(\ell)}$ ) we shall choose.

Since the $\sigma$-algebra $\mathfrak{B}^{(\ell)}$ consists only of unitarily invariant sets, there is no reasonable way to define the integral of functions in $\mathscr{F}_{\ell, 1}$ with respect to spectral measures $E_{\mathrm{T}}$ (because at the starting point, we only know how to integrate the functions $\mathfrak{j}_{\mathscr{B}}$, introduced in Definition 4.4, which belong to $\mathcal{Z}\left(\mathscr{F}_{\ell, 1}\right)$, and there is no reasonable way to approximate functions from $\mathscr{F}_{\ell, 1}$ by functions from $\left.\mathcal{Z}\left(\mathscr{F}_{\ell, 1}\right)\right)$. We shall overcome these difficulties with the aid of the next result. For simplicity, for any Borel subset $\mathscr{B}$ of a Borel kernel of $\mathscr{M}^{(\ell)}$, we shall use $\mathscr{U} . \mathscr{B}$ to denote the set $\left\{U . \mathrm{X}: \mathrm{X} \in \mathscr{B}, U \in \mathscr{U}_{d(\mathrm{X})}\right\}$. The proof of Lemma 2.7 shows that $\mathscr{U} . \mathscr{B} \in \mathfrak{B}^{(\ell)}$ for any such a set $\mathscr{B}$.

Everywhere below, we use $\mathscr{M}^{(*)}$ to denote the $C^{*}$-algebra product of all $\mathscr{M}_{n}$. That is, $\mathscr{M}^{(*)}$ consists of all sequences $\boldsymbol{X}=\left(X_{n}\right)_{n=1}^{\infty}$ with $X_{n} \in \mathscr{M}_{n}$ (for any $n$ ) and $(\|\boldsymbol{X}\| \stackrel{\text { def }}{=}) \sup _{n \geqslant 1}\left\|X_{n}\right\|<\infty$. Each matrix $X \in \mathscr{M}_{n}$ shall be identified with the sequence in $\mathscr{M}^{(*)}$ whose $n$th entry is $X$ and all other entries are zero. One readily checks that under such an identification $\mathscr{M}$ is a topological subspace of $\mathscr{M}^{(*)}$ (that is, the topology of $\mathscr{M}$ coincides with that inherited from $\left.\mathscr{M}^{(*)}\right)$. The unit of $\mathscr{M}^{(*)}$ shall be denoted by $\boldsymbol{E}$.

4.9. Lemma. Let $\mathscr{K}$ be a Borel kernel of $\mathscr{M}^{(\ell)}$ and $\mathrm{T}$ be an $\mathrm{FTI} \ell$-tuple of operators.

(A) The set function $E_{\mathrm{T}}^{\mathscr{K}}: \mathfrak{B}\left(\mathscr{M}^{(\ell)}\right) \rightarrow \mathcal{Z}(\mathcal{W}(\mathrm{T}))$ given by

$$
E_{\mathrm{T}}^{\mathscr{K}}(\mathscr{B}) \stackrel{\text { def }}{=} E_{\mathrm{\top}}(\mathscr{U} \cdot(\mathscr{B} \cap \mathscr{K})) \quad\left(\mathscr{B} \in \mathfrak{B}\left(\mathscr{M}^{(\ell)}\right)\right)
$$

is a spectral measure that extends $E_{\mathrm{T}}$.

(B) The function $\pi_{\mathscr{K}}: \mathscr{M}^{(*)} \rightarrow \mathcal{W}(\mathrm{T})$ given by $\pi_{\mathscr{K}}(\boldsymbol{X}) \stackrel{\text { def }}{=} \hat{c}_{\boldsymbol{X}}[\mathrm{T}]$ is a unital *homomorphism where $\hat{c}_{\boldsymbol{X}}$, for $\boldsymbol{X}=\left(X_{n}\right)_{n=1}^{\infty}$, denotes a unique function in $\mathscr{F}_{\ell, 1}^{b d}$ that is constantly equal to $X_{n}$ on $\mathscr{K} \cap \mathscr{M}_{n}^{\ell}$ for any $n$. 
Proof. To prove (A), it suffices to observe that for any $\mathscr{B}_{1}, \mathscr{B}_{2} \in \mathfrak{B}\left(\mathscr{M}^{(\ell)}\right)$, the intersection of $\mathscr{U} .\left(\mathscr{B}_{1} \cap \mathscr{K}\right)$ and $\mathscr{U} .\left(\mathscr{B}_{2} \cap \mathscr{K}\right)$ coincides with $\mathscr{U} .\left(\mathscr{B}_{1} \cap \mathscr{B}_{2} \cap \mathscr{K}\right)$ (so, in particular, disjoint sets are transformed into disjoint sets), and that $\mathscr{U} \mathscr{B}=\mathscr{B}$ for any $\mathscr{B} \in \mathfrak{B}^{(\ell)}$; whereas the conclusion of $(\mathrm{B})$ follows from Theorem 3.1 and the fact that the assignment $\boldsymbol{X} \mapsto \hat{c}_{\boldsymbol{X}}$ correctly defines a unital $*$-homomorphism of $\mathscr{M}^{(*)}$ into $\mathscr{F}_{\ell, 1}^{b d}$.

Whenever $\mathrm{T}$ is an FTI $\ell$-tuple on a Hilbert space $(\mathcal{H},\langle\cdot,-\rangle)$ and $\mathscr{K}$ is a fixed Borel kernel of $\mathscr{M}^{(\ell)}$, the function $\pi_{\mathscr{K}}$ enables us to define a multiplication of vectors in $\mathcal{H}$ by elements of $\mathscr{M}^{(*)}$. Namely, whenever $h$ is a vector in $\mathcal{H}$ and $\boldsymbol{X}$ is an arbitrary element of $\mathscr{M}^{(*)}$, we may write $\boldsymbol{X} \bullet h$ to denote the vector $\pi^{\mathscr{K}}(\boldsymbol{X}) h$. It follows from Lemma 4.9 that the map $\mathscr{M}^{(*)} \times \mathcal{H} \ni(\boldsymbol{X}, h) \mapsto \boldsymbol{X} \bullet h \in \mathcal{H}$ is bilinear and $\boldsymbol{E} \bullet h=h,(\boldsymbol{X} \boldsymbol{Y}) \bullet h=\boldsymbol{X} \bullet(\boldsymbol{Y} \bullet h)$ and $\left\langle X \bullet h, h^{\prime}\right\rangle=\left\langle h, X^{*} \bullet h^{\prime}\right\rangle$ for any $h, h^{\prime} \in \mathcal{H}$ and $\boldsymbol{X}, \boldsymbol{Y} \in \mathscr{M}^{(*)}$. Such a point of view allows us to look at the Hilbert space $\mathcal{H}$ as a module over $\mathscr{M}^{(*)}$. But then, in a classical way, we may define integration of $\mathscr{M}^{(*)}$-valued functions with respect to $\mathcal{B}(\mathcal{H})$-valued spectral measures. The concept of integrating vector-valued maps with respect to vector-valued measures (starting from a bilinear map) is well developed; see, for example, a book [10] or a pioneering paper [1] on this topic as well as further [18, 19, 33, [52, 49] and [41] (consult also [9] or Chapter IV in [12]).

To define integration, we fix a Borel kernel $\mathscr{K}$ of $\mathscr{M}^{(\ell)}$ and an FTI $\ell$-tuple T of operators in a Hilbert space $\mathcal{H}$, and put, for simplicity, $E \stackrel{\text { def }}{=} E_{\mathrm{T}}^{\mathscr{K}}$. Also, to make the presentation more transparent, we shall use the notation " $\boldsymbol{X} \bullet h$ " introduced above (where $\boldsymbol{X} \in \mathscr{M}^{(*)}$ and $h \in \mathcal{H}$ ) instead of $\pi_{\mathscr{K}}(\boldsymbol{X}) h$. Since $E$ takes values in the center of $\mathcal{W}(T)$, we obtain

$$
E(\sigma)(\boldsymbol{X} \bullet h)=\boldsymbol{X} \bullet(E(\sigma) h) \quad\left(\sigma \in \mathfrak{B}\left(\mathscr{M}^{(\ell)}\right), \boldsymbol{X} \in \mathscr{M}^{(*)}, h \in \mathcal{H}\right),
$$

which means that the operators $E(\sigma)$ are $\mathscr{M}^{(*)}$-linear (when thinking of $\mathcal{H}$ as an $\mathscr{M}^{(*)}$-module). This remark makes the concept of integration defined below more natural and typical.

First assume a Borel function $v: \mathscr{M}^{(\ell)} \rightarrow \mathscr{M}^{(*)}$ is bounded and has a countable range. We claim that then the series $\sum_{\boldsymbol{X} \in \mathscr{M}^{(*)}} \boldsymbol{X} \bullet E\left(v^{-1}(\{\boldsymbol{X}\})\right)$ is unconditionally convergent in the strong operator topology. Indeed, we may assume the range of $v$ is infinite and arrange all its elements in a one-to-one sequence $\boldsymbol{X}_{1}, \boldsymbol{X}_{2}, \ldots$ Further, put $\sigma_{n}=\mathscr{U} \cdot\left(v^{-1}\left(\left\{\boldsymbol{X}_{n}\right\}\right) \cap \mathscr{K}\right)$. For any $\boldsymbol{X} \in \mathscr{M}^{(*)}$, let $\hat{c}_{\boldsymbol{X}}$ be as specified in part (B) of Lemma 4.9. Observe that the sets $\sigma_{n}$ are pairwise disjoint and

$$
\sum_{\boldsymbol{X} \in \mathscr{M}^{(*)}} \boldsymbol{X} \cdot E\left(v^{-1}(\{\boldsymbol{X}\})\right)=\sum_{n=1}^{\infty}\left(\mathfrak{j}_{\sigma_{n}} \hat{c}_{\boldsymbol{X}_{n}}\right)[\mathrm{T}] .
$$

So, since the series $\sum_{n=1}^{\infty} \mathfrak{j}_{\sigma_{n}} \hat{c}_{\boldsymbol{X}_{n}}$ is pointwise convergent to a function in $\mathscr{F}_{\ell, 1}^{b d}$ and its partial sums are uniformly bounded (because $v$ is bounded), we conclude that the right-hand side of the above formula converges (unconditionally) in the strong operator topology (by (F5)). We define $\int_{\mathscr{M}^{(\ell)}}^{\pi_{\mathscr{K}}} v \mathrm{~d} E_{\mathrm{T}}^{\mathscr{K}}$ as the limit of the aforementioned series. Note that

$$
\int_{\mathscr{M}^{(\ell)}}^{\pi_{\mathscr{K}}} v \mathrm{~d} E_{\mathrm{T}}^{\mathscr{K}}=\tilde{v}[\mathrm{~T}]
$$

where $\tilde{v} \in \mathscr{F}_{\ell, 1}^{b d}$ is given by $\tilde{v}(\mathbf{X}) \stackrel{\text { def }}{=} \sum_{\boldsymbol{X} \in \mathscr{M}^{(*)}} \mathfrak{j}_{\mathscr{U}} \cdot\left(v^{-1}(\{\boldsymbol{X}\}) \cap \mathscr{K}\right)(\mathrm{X}) \hat{c}_{\boldsymbol{X}}(\mathrm{X})$. It is easy to check that $\tilde{v}(\mathrm{X})$ coincides with the $n$th entry of $v(\mathrm{X})$ for any $\mathrm{X} \in \mathscr{K} \cap \mathscr{M}_{n}^{\ell}$. This implies that the assignment $v \mapsto \tilde{v}$ (when $v$ runs over all Borel functions with countable ranges) defines a continuous unital $*$-homomorphism $\Phi_{0}$ whose norm does not exceed 1 . Consequently, also the function $\Psi_{0}$ which assigns to each such a 
function $v$ the integral $\int_{\mathscr{M}^{(\ell)}}^{\pi_{\mathscr{K}}} v \mathrm{~d} E_{\mathrm{T}}^{\mathscr{K}}$ is a unital $*$-homomorphism and $\left\|\Psi_{0}\right\| \leqslant 1$ (by (4-4) ). So, both $\Phi_{0}$ and $\Psi_{0}$ extend to unital $*$-homomorphisms $\Phi$ and $\Psi$ defined on the uniform closure $\mathcal{D}$ of their common domain $\mathcal{D}_{0}$. It is well-known (and follows, for example, from a classical theorem due to Pettis 44) that $\mathcal{D}$ consists of all functions $f: \mathscr{M}^{(\ell)} \rightarrow \mathscr{M}^{(*)}$ for which $f\left(\mathscr{M}^{(\ell)}\right.$ is a bounded separable subspace of $\mathscr{M}^{(*)}$ and $\psi \circ f: \mathscr{M}^{(\ell)} \rightarrow \mathbb{C}$ is Borel for any continuous linear functional $\psi$ on $\mathscr{M}^{(*)}$. (It follows from theory of Suslin spaces that the image in a metrizable space of a Polish space under a Borel function is always separable. Thus, $\mathcal{D}$ constists precisely of all bounded Borel functions from $\mathscr{M}^{(\ell)}$ into $\mathscr{M}^{(*)}$.) Since $\Psi_{0}$ is $\mathscr{M}^{(*)}$ linear (that is, $\Psi_{0}(\boldsymbol{X} \bullet v)=\boldsymbol{X} \bullet \Psi_{0}(v)$ for any $v \in \mathcal{D}_{0}$ and $\boldsymbol{X} \in \mathscr{M}^{(*)}$, which may be easily verified), we infer that also $\Psi$ is $\mathscr{M}^{(*)}$-linear. Finally, denoting by $\tilde{v}$ and $\int_{\mathscr{M}^{(\ell)}}^{\pi_{\mathscr{K}}} v \mathrm{~d} E_{\mathrm{T}}^{\mathscr{K}}$ the values at $v \in \mathcal{D}$ of, respectively, $\Phi$ and $\Psi$, equation (4-4) still hold (for all $v \in \mathcal{D}$ ).

To reduce the number of symbols, let us simplify a suggestive notation for the integral in (4-4) and write $\int^{\mathscr{K}} v \mathrm{~d} E_{\mathrm{T}}$ or $\int^{\mathscr{K}} v(\mathrm{X}) \mathrm{d} E_{\mathrm{T}}(\mathrm{X})$ instead.

4.10. Proposition. For any Borel kernel $\mathscr{K}$ and $u \in \mathscr{F}_{\ell, 1}^{b d}$,

$$
\int^{\mathscr{K}} u \mathrm{~d} E_{\mathrm{T}}=u[\mathrm{~T}]
$$

Proof. We know that $\int^{\mathscr{K}} u \mathrm{~d} E_{\mathrm{T}}=\tilde{u}[\mathrm{~T}]$. Thus, it suffices to check that $\tilde{u}=u$ (for $\left.u \in \mathscr{F}_{\ell, 1}\right)$. To this end, recall that for any $v \in \mathcal{D}_{0}, \tilde{v}(\mathrm{X})$ coincides with the $n$th entry of $v(\mathrm{X})$ for all $\mathrm{X} \in \mathscr{K} \cap \mathscr{M}_{n}^{\ell}$. Consequently, the same can be said about each $v \in \mathcal{D}$. So, we conclude that for any $u \in \mathscr{F}_{\ell, 1}^{b d}, \tilde{u}$ and $u$ coincide on $\mathscr{K}$ and hence $\tilde{u}=u$.

Proposition 4.10 implies that $\int^{\mathscr{K}} v \mathrm{~d} E_{\mathrm{T}}=\int^{\mathscr{K}} \tilde{v} \mathrm{~d} E_{\mathrm{T}}$ for each $v \in \mathcal{D}$. So, the value of the integral always belongs to $\mathcal{W}^{\prime \prime}(T)$. One may also readily derive from the above property bounded convergence theorem which asserts that $\int^{\mathscr{K}} v_{n} \mathrm{~d} E_{\mathrm{T}}$ converge in the $*$-strong operator topology to $\int^{\mathscr{K}} v \mathrm{~d} E_{\mathrm{T}}$ provided $v_{n} \in \mathcal{D}$ are uniformly bounded and converge pointwise to $v \in \mathcal{D}$.

For unbounded Borel functions $u: \mathscr{M}^{(\ell)} \rightarrow \mathscr{M}^{(*)}$ (they automatically have separable ranges), the integral $\int^{\mathscr{K}} u \mathrm{~d} E_{\mathrm{T}}$ may be defined as an operator in $\hat{\mathcal{W}}$ where $\mathcal{W}=\mathcal{W}^{\prime \prime}(\mathrm{T})$ as follows. (We argue similarly as in the proof of Proposition 3.11.) There exists a sequence $\mathscr{B}_{1}, \mathscr{B}_{2}, \infty$ of pairwise disjoint Borel sets that cover $\mathscr{M}^{(\ell)}$ and $u$ is bounded on each of them. Let $b_{n}: \mathscr{M}^{(\ell)} \rightarrow \mathscr{M}^{(*)}$ denote the characteristic function of $\mathscr{B}_{n}$. We define $\int^{\mathscr{K}} u \mathrm{~d} E_{\mathrm{T}}$ as $\sum_{n=1}^{\infty} \int^{\mathscr{K}} u b_{n} \mathrm{~d} E_{\mathrm{T}}$. Although such a definition is quite natural, it is not obvious that the value of $\int^{\mathscr{K}} u \mathrm{~d} E_{\mathrm{T}}$ is independent of the choice of the sets $\mathscr{B}_{n}$. We shall check it with the aid of (4-4). We know from that formula that $\int^{\mathscr{K}} u b_{n} \mathrm{~d} E_{\mathrm{T}}=\widetilde{u b_{n}}[\mathrm{~T}]$ for any $n$. It may be easily checked that $\widetilde{u b_{n}}$ coincides with $\mathfrak{j}_{\mathscr{U}} .\left(\mathscr{B}_{n} \cap \mathscr{K}\right) \tilde{u}$ (where, as before, $\tilde{u}$ is a unique function $f \in \mathscr{F}_{\ell, 1}$ such that for any $\mathrm{X} \in \mathscr{K} \cap \mathscr{M}_{n}^{\ell}, f(\mathrm{X})$ is the $n$th entry of $\left.u(\mathrm{X})\right)$. So, $\widetilde{u b_{n}}[\mathrm{~T}]=\left(\mathbf{j}_{\mathscr{U}}\left(\mathscr{B}_{n} \cap \mathscr{K}\right) \tilde{u}\right)[\mathrm{T}] \cdot \mathbf{j}_{\mathscr{U}} \cdot\left(\mathscr{B}_{n} \cap \mathscr{K}\right)[\mathrm{T}]$. Observing that the functions $\mathbf{j}_{\mathscr{U}}\left(\mathscr{B}_{n} \cap \mathscr{K}\right)$ are mutually disjoint and sum up to the unit matrix of a respective degree at each irreducible $\ell$-tuple of matrices, we infer from the very definition of $\tilde{u}[\mathbf{T}]$ that

$$
\int^{\mathscr{K}} u \mathrm{~d} E_{\mathrm{T}}=\sum_{n=1}^{\infty}\left(\mathrm{j}_{\mathscr{U} .\left(\mathscr{B}_{n} \cap \mathscr{K}\right)} \tilde{u}\right)[\mathrm{T}] \mathrm{j}_{\mathscr{U} .\left(\mathscr{B}_{n} \cap \mathscr{K}\right)}[\mathrm{T}]=\tilde{u}[\mathrm{~T}] .
$$

In particular, the above integral is well defined, (4-4) holds for any Borel function $u: \mathscr{M}^{(\ell)} \rightarrow \mathscr{M}^{(*)}$ and, consequently, (4-5) holds for any $u \in \mathscr{F}_{\ell, 1}$. 
Finally, if, for a Borel function $u: \mathscr{M}^{(\ell)} \rightarrow\left(\mathscr{M}^{(*)}\right)^{\ell^{\prime}}$, we define $\int^{\mathscr{K}} u \mathrm{~d} E_{\mathrm{\top}}$ coordinatewise, that is,

$$
\int^{\mathscr{K}} u \mathrm{~d} E_{\mathrm{T}} \stackrel{\text { def }}{=}\left(\int^{\mathscr{K}} \pi_{1} \circ u \mathrm{~d} E_{\mathrm{T}}, \ldots, \int^{\mathscr{K}} \pi_{\ell^{\prime}} \circ u \mathrm{~d} E_{\mathrm{T}}\right)
$$

(where $\pi_{k}:\left(\mathscr{M}^{(*)}\right)^{\ell^{\prime}} \rightarrow \mathscr{M}^{(*)}$ is the natural projection onto the $k$ th coordinate), then we obtain

4.11. Theorem (Spectral Theorem). Let T be an FTI $\ell$-tuple of operators. For any Borel kernel $\mathscr{K}$ of $\mathscr{M}^{(\ell)}$,

$$
\int^{\mathscr{K}} \mathrm{X} \mathrm{d} E_{\mathrm{T}}(\mathrm{X})=\mathrm{T}
$$

Now we shall discuss how the multiplication "•" (by elements of $\mathscr{M}^{(*)}$ ) changes when $\mathscr{K}$ varies. To distinguish between such multiplications induced by different Borel kernels, below we write $\boldsymbol{X} \bullet_{\mathscr{K}} h$ to denote $\pi_{\mathscr{K}}(\boldsymbol{X}) h$.

4.12. Proposition. Let $\mathscr{K}$ and $\mathscr{K}^{\prime}$ be two Borel kernels of $\mathscr{M}^{(\ell)}$. Then there exists a function $u \in \mathscr{F}_{\ell, 1}^{b d}$ such that:

- $u$ is a unitary element of $\mathscr{F}_{\ell, 1}^{b d}$, that is, $u^{*} u=u u^{*}=\mathfrak{j}$; and

- the function $\phi: \mathscr{M}^{(\ell)} \ni \mathrm{X} \mapsto u(\mathrm{X}) . \mathrm{X} \in \mathscr{M}^{(\ell)}$ is a well defined unitary element of $\mathscr{F}_{\ell, \ell}^{\text {loc }}$ as well as a Borel isomorphism that sends $\mathscr{K}$ onto $\mathscr{K}^{\prime}$;

and for any $\mathrm{FTI} \ell$-tuple $\mathrm{T}$ of operators in $\mathcal{H}$ :

- $E_{\mathrm{T}}^{\mathscr{K}^{\prime}}(\sigma)=E_{\mathrm{T}}^{\mathscr{K}}\left(\phi^{-1}(\sigma)\right)$ for each $\sigma \in \mathfrak{B}\left(\mathscr{M}^{(\ell)}\right)$ (that is, $E_{\mathrm{T}}^{\mathscr{K}^{\prime}}$ is the transport of $E_{\top}^{\mathscr{K}}$ under $\left.\phi\right)$; and

- $\boldsymbol{X} \bullet \mathscr{K}^{\prime} h=u[\mathrm{~T}]\left(\boldsymbol{X} \cdot \mathscr{K}\left(u^{*}[\mathrm{~T}] h\right)\right)$ for any $\boldsymbol{X} \in \mathscr{M}^{(*)}$ and $h \in \mathcal{H}$ (that is, the multiplication by $\boldsymbol{X}$ induced by $\mathscr{K}^{\prime}$ is unitarily equivalent to that induced by $\mathscr{K})$.

It is worth noting here that the connection between the integrals $\int^{\mathscr{K}} u \mathrm{~d} E_{\mathrm{T}}$ and $\int^{\mathscr{K}^{\prime}} u \mathrm{~d} E_{\mathrm{\top}}$ for a Borel function $u: \mathscr{M}^{(\ell)} \rightarrow \mathscr{M}^{(*)}$ is, in general, hard to describe (but, for $u \in \mathscr{F}_{\ell, 1}$, both these integrals coincide, which follows from Proposition 4.10 and the remarks preceding Theorem 4.11).

Proof. As shown in the proof of Lemma 2.8, there is a Borel function $g: \mathscr{K} \rightarrow \mathscr{M}$ such that $g(\mathrm{X}) \in \mathscr{U}_{d(\mathrm{X})}$ and $g(\mathrm{X}) . \mathrm{X} \in \mathscr{K}^{\prime}$ for any $\mathrm{X} \in \mathscr{K}$. It follows from Lemma 2.8 that $g$ extends to a function $u \in \mathscr{F}_{\ell, 1}^{b d}$. Then one deduces that $u(\mathrm{X}) \in \mathscr{U}_{d(\mathrm{X})}$ for each $\mathrm{X} \in \mathscr{M}^{(\ell)}$ and, consequently, $u^{*} u=u u^{*}=\mathrm{j}$. Further, straightforward calculations show that the function $\phi: \mathscr{M}^{(\ell)} \rightarrow \mathscr{M}^{(\ell)}$ defined by $\phi(\mathrm{X}) \stackrel{\text { def }}{=} u(\mathrm{X}) . \mathrm{X}$ is compatible. It is also easily seen that $\phi$ is locally bounded and Borel, and $\phi(\mathscr{K})=\mathscr{K}^{\prime}$. So, we infer from the compatibility of $\phi$ that $\phi\left(\mathscr{M}^{(\ell)}\right)=\mathscr{M}^{(\ell)}$. Thus, to conclude that $\phi$ is a Borel isomorphism, it suffices to check that it is one-to-one (again, by Suslin's theorem). To this end, assume $\phi(\mathrm{X})=\phi(\mathrm{Y})$. Then $d(\mathrm{X})=d(\mathrm{Y})$ and

$$
u(\mathrm{X}) \cdot \mathrm{X}=u(\mathrm{Y}) \cdot \mathrm{Y} \text {. }
$$

Taking into account the facts that $u$ is compatible and both $u(\mathbf{X})$ and $u(\mathrm{Y})$ are unitary, (4-6) yields that $(u(\mathbf{X})=) u(\mathbf{X}) \cdot u(\mathbf{X})=u(u(\mathbf{X}) \cdot \mathbf{X})=u(u(\mathbf{Y}) \cdot \mathrm{Y})=u(\mathbf{Y}) \cdot u(\mathbf{Y})(=$ $u(\mathrm{Y}))$, and hence $\mathrm{X}=\mathrm{Y}($ by (4-6) $)$.

Now let T be an FTI $\ell$-tuple of operators in $\mathcal{H}$. For any $\sigma \in \mathfrak{B}\left(\mathscr{M}^{(\ell)}\right)$, the compatibility of $\phi$ implies that $\mathscr{U} .\left(\sigma \cap \mathscr{K}^{\prime}\right)=\mathscr{U} \cdot \phi^{-1}\left(\sigma \cap \mathscr{K}^{\prime}\right)=\mathscr{U} \cdot\left(\phi^{-1}(\sigma) \cap \mathscr{K}\right)$ and hence $E_{\mathrm{T}}^{\mathscr{K}^{\prime}}(\sigma)=E_{\mathrm{T}}^{\mathscr{K}}\left(\phi^{-1}(\sigma)\right)$. Finally, to establish the connection between the multiplications (by elements of $\mathscr{M}^{(*)}$ ) induced by $\mathscr{K}$ and $\mathscr{K}^{\prime}$, for any $\boldsymbol{X} \in$ $\mathscr{M}^{(*)}$, we shall use $\hat{c}_{\boldsymbol{X}}$ and $\hat{c}_{\boldsymbol{X}}^{\prime}$ to denote the functions in $\mathscr{F}_{\ell, 1}^{b d}$ that are constantly 
equal to the $n$th coordinate of $\boldsymbol{X}$ on, respectively, $\mathscr{K} \cap \mathscr{M}_{n}^{\ell}$ and $\mathscr{K}^{\prime} \cap \mathscr{M}_{n}^{\ell}$ (see part (B) of Lemma 4.9). Then, for any $Y \in \mathscr{K}$ and $\boldsymbol{X}=\left(X_{n}\right)_{n=1}^{\infty} \in \mathscr{M}^{(*)}$, $\hat{c}_{\boldsymbol{X}}^{\prime}(\phi(\mathrm{Y}))=X_{d(\mathrm{Y})}=\hat{c}_{\boldsymbol{X}}(\mathrm{Y})$. So, since both $\hat{c}_{\boldsymbol{X}}^{\prime} \circ \phi$ and $\hat{c}_{\boldsymbol{X}}$ belong to $\mathscr{F}_{\ell, 1}$ and coincide on $\mathscr{K}$, we conclude that $\hat{c}_{\boldsymbol{X}}^{\prime} \circ \phi=\hat{c}_{\boldsymbol{X}}$. Consequently, for any $h \in \mathcal{H}$, $\boldsymbol{X} \cdot \mathscr{K}^{\prime} h=\pi_{\mathscr{K}^{\prime}}(\boldsymbol{X}) h=\left(\hat{c}_{\boldsymbol{X}} \circ \phi\right)[\mathrm{T}] h=\hat{c}_{\boldsymbol{X}}[\phi[\mathrm{T}]] h$. Further, observe that (by definition) $\phi=\left(u \pi_{1}^{(\ell)}(u)^{-1}, \ldots, u \pi_{\ell}^{(\ell)}(u)^{-1}\right)$ and, consequently,

$$
\phi[\mathrm{T}]=\left(u[\mathrm{~T}] \pi_{1}^{(\ell)}[\mathrm{T}](u)^{-1}[\mathrm{~T}], u[\mathrm{~T}] \pi_{\ell}^{(\ell)}[\mathrm{T}](u)^{-1}[\mathrm{~T}]\right)=u[\mathrm{~T}] . \mathrm{T}
$$

(recall that $u[\mathrm{~T}]$ is a unitary operator). Further, we infer from Corollary 3.12 that $\hat{c}_{\boldsymbol{X}}[u[\mathrm{~T}] . \mathrm{T}]=u[\mathrm{~T}] . \hat{c}_{\boldsymbol{X}}[\mathrm{T}]=u[\mathrm{~T}] \hat{c}_{\boldsymbol{X}}[\mathrm{T}] u^{*}[\mathrm{~T}]$. All these remarks yield $\boldsymbol{X} \bullet_{\mathscr{K}^{\prime}} h=$ $u[\mathrm{~T}] \hat{c}_{\boldsymbol{X}} u^{*}[\mathrm{~T}] h=u[\mathrm{~T}]\left(\boldsymbol{X} \bullet \mathscr{K}\left(u^{*}[\mathrm{~T}] h\right)\right)$ and we are done.

The above result asserts that all operators of multiplication by a fixed element of $\mathscr{M}^{(*)}$ induced by Borel kernels of $\mathscr{M}^{(\ell)}$ are mutually unitarily equivalent. In general, there is no reasonable way to distinguish one of them as best fitting to a fixed FTI $\ell$-tuple. This causes that many attributes (such as principal spectrum) of such tuples have to be unitarily invariant. This is one of basic differences between general FTI tuples and normal operators. For the latters, their principal spectra coincide with usual ("algebraic"). The next result establishes a counterpart of that property for general FTI tuples, where the operator of multiplication of vectors in $\mathcal{H}$ by $\boldsymbol{X} \in \mathscr{M}^{(*)}$ induced by a Borel kernel $\mathscr{K}$ shall be suggestively denoted by $\boldsymbol{X} \bullet I_{\mathcal{H}}$ (here and below, $I_{\mathcal{H}}$ is the identity operator on $\mathcal{H}$ ).

4.13. Proposition. Let $\mathrm{T}=\left(T_{1}, \ldots, T_{\ell}\right)$ be an $\mathrm{FTI} \ell$-tuple of operators in a separable Hilbert space $\mathcal{H}$ and $\mathrm{X}=\left(X_{1}, \ldots, X_{\ell}\right)$ be an irreducible $\ell$-tuple in $\mathscr{M}_{p}^{\ell}$. The following conditions are equivalent:

(i) $\mathrm{X}$ belongs to the principal spectrum of $\mathrm{T}$;

(ii) there exists a Borel kernel $\mathscr{K}$ of $\mathscr{M}^{(\ell)}$ such that for any $\varepsilon>0$ there is a nonzero projection $Z \in \mathcal{Z}\left(\mathcal{W}^{\prime \prime}(\mathrm{T})\right)$ with $Z \mathcal{W}^{\prime \prime}(\mathrm{T})$ type $\mathrm{I}_{p}$ and

$$
\left\|\left(T_{k}-X_{k} \cdot I_{\mathcal{H}}\right) \cdot Z\right\| \leqslant \varepsilon \quad(k=1, \ldots, \ell) .
$$

Proof. Assume $\mathscr{K}$ is as specified in (ii). To prove (i), we need to check that $E_{\mathrm{T}}(\mathscr{B}) \neq 0$ for any set $\mathscr{B} \in \mathfrak{B}^{(\ell)}$ which contains $\mathrm{X}$ and is relatively open in $\mathscr{I}^{(\ell)}$. To this end, fix such a set $\mathscr{B}$. Then there is a number $\delta>0$ such that each $\mathrm{Y} \in \mathscr{M}_{d(\mathrm{X})}^{\ell}$ with $\|\mathrm{Y}-\mathrm{X}\| \leqslant \delta$ belongs to $\mathscr{B}$ (we use here the property that $\mathscr{I}^{(\ell)}$ is open in $\left.\mathscr{M}^{(\ell)}\right)$. Using (ii), take a nonzero projection $Z \in \mathcal{Z}\left(\mathcal{W}^{\prime \prime}(\mathrm{T})\right.$ ) for which $Z \mathcal{W}^{\prime \prime}(\mathrm{T})$ is type $\mathrm{I}_{p}$ and (4-7) holds with $\varepsilon=\delta$. One deduces from Proposition 3.6 and Corollary 4.6 that there is $b \in \mathcal{Z}\left(\mathscr{F}_{\ell, 1}^{b d}\right)$ with $b^{*} b=\mathfrak{j}$ and $b[\mathrm{~T}]=Z$. Moreover, since $Z \mathcal{W}^{\prime \prime}(\mathrm{T})$ is type $\mathrm{I}_{p}$, we may and do assume that $b$ vanishes at each point of $\mathscr{I}^{(\ell)} \backslash \mathscr{M}_{p}^{\ell}$ (thanks to Proposition 4.1).

Recall that $X_{k} \cdot I_{\mathcal{H}}=\hat{c}_{X_{k}}[\mathrm{~T}]$ and hence $\left(T_{k}-X_{k} \cdot I_{\mathcal{H}}\right) \cdot Z=w_{k}[\mathrm{~T}]$ where $w_{k} \stackrel{\text { def }}{=}\left(\pi_{k}^{(\ell)}-\hat{c}_{X_{k}}\right) b$. Further, Proposition 3.6 and (4-7) imply that $\left(T_{k}-X_{k}\right.$ • $\left.I_{\mathcal{H}}\right) \cdot Z=v_{k}[\mathrm{~T}]$ for some $v_{k} \in \mathscr{F}_{\ell, 1}^{b d}$ with $\left\|v_{k}\right\| \leqslant \delta$. So, $w_{k}[\mathrm{~T}]=v_{k}[\mathrm{~T}]$; and Corollary 4.6 asserts that $E_{\mathrm{T}}\left(D\left(v_{k}, w_{k}\right)\right)=0$. We conclude that $E_{\mathrm{T}}\left(\mathscr{Z}_{k}\right)=I_{\mathcal{H}}$ where $\mathscr{Z}_{k} \stackrel{\text { def }}{=}\left\{\mathrm{Y} \in \mathscr{I}^{(\ell)}:\left\|w_{k}(\mathrm{Y})\right\| \leqslant \delta\right\}$. Further, since $Z=b[\mathrm{~T}]$ is nonzero, the value of $E_{\mathrm{T}}$ at $\mathscr{Z}_{0} \stackrel{\text { def }}{=}\left\{\mathrm{Y} \in \mathscr{I}^{(\ell)}: b(\mathrm{Y})=I_{d(\mathrm{Y})}\right\}$ is nonzero as well. Consequently, $E_{\mathrm{T}}(\mathscr{Z}) \neq 0$ where $\mathscr{Z} \stackrel{\text { def }}{=} \bigcap_{k=0}^{\ell} \mathscr{Z}_{k}$. Now let $\mathrm{Y} \in \mathscr{Z} \cap \mathscr{K}$ and $k \in\{1, \ldots, \ell\}$. Then $\left\|\pi_{k}^{(\ell)}(\mathrm{Y})-\hat{c}_{X_{k}}(\mathrm{Y})\right\|=\left\|w_{k}(\mathrm{Y})\right\| \leqslant \delta$. But $\mathscr{Z}_{0} \subset \mathscr{M}_{p}^{\ell}$ and therefore $\hat{c}_{X_{k}}(\mathrm{Y})=X_{k}$. So, $\|\mathrm{Y}-\mathrm{X}\| \leqslant \delta$, which gives $\mathrm{Y} \in \mathscr{B}$ (for any $\mathrm{Y} \in \mathscr{Z} \cap \mathscr{K}$ ). We conclude that $\mathscr{U} .(\mathscr{Z} \cap \mathscr{K}) \subset \mathscr{B}$. Finally, since $\mathscr{Z}$ is unitarily invariant, we see that $\mathscr{U}$. $(\mathscr{Z} \cap \mathscr{K})=$ $\mathscr{Z}$ and hence $E_{\mathrm{T}}(\mathscr{B}) \neq 0$. 
Now assume $\boldsymbol{X} \in \operatorname{supp}\left(E_{\mathrm{T}}\right)$. We shall construct a Borel kernel $\mathscr{K}$ for which (ii) holds. To this end, we fix an arbitrary Borel kernel $\mathscr{K}^{\prime}$ and define the function $\xi: \mathscr{K}^{\prime} \cap \mathscr{M}_{p}^{\ell} \rightarrow \mathbb{R}$ by $\xi(\mathrm{Y}) \stackrel{\text { def }}{=} \min \left\{\|\mathrm{X}-U . \mathrm{Y}\|: U \in \mathscr{U}_{p}\right\}$. It is easy to check that $\xi$ is continuous. Further, let $\Xi$ be a multifunction on $\mathscr{K}^{\prime} \cap \mathscr{M}_{p}^{\ell}$ defined by $\Xi(\mathrm{Y}) \stackrel{\text { def }}{=}\left\{U \in \mathscr{U}_{p}:\|\mathrm{X}-U . \mathrm{Y}\|=\xi(\mathrm{Y})\right\}$ (for $Y \in \mathscr{K}^{\prime} \cap \mathscr{M}_{p}^{\ell}$ ). Notice that for any compact set $\mathscr{L} \subset \mathscr{U}_{p}$, the set $\left\{\mathrm{Y} \in \mathscr{K}^{\prime} \cap \mathscr{M}_{p}^{\ell}: \Xi(\mathrm{Y}) \cap \mathscr{L} \neq \varnothing\right\}$ is relatively closed in $\mathscr{K}^{\prime} \cap \mathscr{M}_{p}^{\ell}$ and, consequently, $\left\{\mathrm{Y} \in \mathscr{K}^{\prime} \cap \mathscr{M}_{p}^{\ell}: \Xi(\mathrm{Y}) \cap \mathscr{G} \neq \varnothing\right\} \in \mathfrak{B}\left(\mathscr{K}^{\prime} \cap \mathscr{M}_{p}^{\ell}\right)$ for any set $\mathscr{G} \subset \mathscr{U}_{p}$ that is relatively open in $\mathscr{U}_{p}$. So, it follows from the KuratowskiRyll-Nardzewski selection theorem [31] (see also Theorem 1 in $\S 1$ of Chapter XIV in [30]) that there exists a Borel function $\tau: \mathscr{K}^{\prime} \cap \mathscr{M}_{p}^{\ell} \rightarrow \mathscr{U}_{p}$ such that $\tau(\mathrm{Y}) \in \Xi(\mathrm{Y})$ for any $\mathrm{Y} \in \mathscr{K}^{\prime} \cap \mathscr{M}_{p}^{\ell}$. This means that

$$
\|\mathrm{X}-\tau(\mathrm{Y}) . \mathrm{Y}\|=\min \left\{\|\mathrm{X}-U . \mathrm{Y}\|: U \in \mathscr{U}_{p}\right\} \quad\left(\mathrm{Y} \in \mathscr{K}^{\prime} \cap \mathscr{M}_{p}^{\ell}\right) .
$$

We define $\mathscr{K}$ as $\left(\mathscr{K}^{\prime} \backslash \mathscr{M}_{p}^{\ell}\right) \cup\left\{\tau(\mathrm{Y}) . \mathrm{Y}: \mathrm{Y} \in \mathscr{K}^{\prime} \cap \mathscr{M}_{p}^{\ell}\right\}$. It is immediate that $\mathscr{K}$ is a kernel of $\mathscr{M}^{\ell}$. The property that $\mathscr{K}$ is Borel follows from the Suslin theorem (on the ranges of one-to-one Borel functions). We shall now check that $\mathscr{K}$ is the kernel we searched for. To this end, fix $\varepsilon>0$ and denote by $\mathscr{B}_{0}$ the set of all $\mathrm{Y} \in \mathscr{K} \cap \mathscr{M}_{p}^{\ell}$ with $\|\mathrm{X}-\mathrm{Y}\| \leqslant \varepsilon$. We claim that $\mathscr{B} \stackrel{\text { def }}{=} \mathscr{U} \cdot \mathscr{B}_{0}\left(\in \mathfrak{B}^{(\ell)}\right)$ is a neighbourhood of $\mathrm{X}$ (not necessarily open). Indeed, if $\delta<\varepsilon$ is a positive real number such that the open ball $\mathscr{S}$ around $\mathrm{X}$ of radius $\delta$ is contained in $\mathscr{I}^{(\ell)}$, then $\mathscr{S} \subset \mathscr{B}$, which readily follows from (4-8). So, since $X \in \operatorname{supp}\left(E_{\mathrm{T}}\right)$, we infer that $E_{\mathrm{T}}(\mathscr{B}) \neq 0$, and hence $Z \stackrel{\text { def }}{=} \mathrm{j}_{\mathscr{B}}[\mathrm{T}]$ is nonzero, by Corollary 4.6 . We know that $Z \in \mathcal{Z}\left(\mathcal{W}^{\prime \prime}(\mathrm{T})\right)$ is a projection such that $Z \mathcal{W}^{\prime \prime}(\mathrm{T})$ is type $\mathrm{I}_{p}$ (thanks to Proposition 4.1). Thus, it remains to verify (4-7). To this end, fix $k \in\{1, \ldots, \ell\}$, put $w \stackrel{\text { def }}{=}\left(\pi_{k}^{(\ell)}-\hat{c}_{X_{k}}\right) j_{\mathscr{B}}$ and note that $\left(T_{k}-X_{k} \bullet I_{\mathcal{H}}\right) \cdot Z=w[\mathrm{~T}]$. So, to convince oneself that (4-7) holds, it is enough to show that $\|w\| \leqslant \varepsilon$ or, equivalently, that $\|w(\mathrm{Y})\| \leqslant \varepsilon$ for any $\mathrm{Y} \in \mathscr{K}$ (see Corollary 2.5). Fix such $\mathrm{Y}$. Since $j_{\mathscr{B}}(\mathrm{Y})=0$ for $\mathrm{Y} \notin \mathscr{B}$, we may assume $\mathrm{Y} \in \mathscr{B}$. Observe that $\mathscr{B} \cap \mathscr{K}=\mathscr{B}_{0}$ and hence $\|\mathrm{X}-\mathrm{Y}\| \leqslant \varepsilon$. Further, $\hat{c}_{X_{k}}(\mathrm{Y})=X_{k}$ and therefore $\|w(\mathbf{Y})\|=\left\|\pi_{k}^{(\ell)}(\mathbf{Y})-X_{k}\right\| \leqslant\|\mathbf{Y}-\mathbf{X}\| \leqslant \varepsilon$, and we are done.

We conclude the paper with the next result, which describes a method of producing FTI tuples. For its purposes, let us agree that whenever $T_{j k}$, for $j, k=1, \ldots, N$, are operators in a common Hilbert space $\mathcal{H}$, then $\left[T_{j k}\right]$ will denote the operator $S$ in $\mathcal{H}^{N}$ defined as follows. The domain of $S$ consists of all vectors $\left(h_{1}, \ldots, h_{N}\right) \in \mathcal{H}^{N}$ such that $h_{k}$ belongs to the domain of $T_{j k}$ for any $j$ and $k$; and for $h=\left(h_{1}, \ldots, h_{N}\right)$ in the domain of $S, S h$ is computed in a standard manner, as the multiplication of two matrices (of course, one needs to write $h$ as a column). The operator $\left[T_{j k}\right]$ may not be neither closed nor densely defined.

4.14. Proposition. If all operators $T_{j k}^{(p)}$ (where $p=1, \ldots, \ell$ and $\left.j, k=1, \ldots, N\right)$ are affiliated with a common finite type I von Neumann algebra, then the operators $S_{p} \stackrel{\text { def }}{=}\left[T_{j k}^{(p)}\right](p=1, \ldots, \ell)$ are densely defined and closable and the $\ell$-tuple of their closures is $\mathrm{FTI}$.

Proof. Let $\mathcal{H}$ and $\mathcal{M}$ denote the Hilbert space which all operators $T_{j k}^{(p)}$ act in and, respectively, a finite type I von Neumann algebra which all these operators are affiliated with. Let $\mathcal{W}$ denote the von Neumann algebra of all operators on $\mathcal{H}^{N}$ of the form $\left[S_{j k}\right]$ where all $S_{j k}$ belong to $\mathcal{M}$. Since $\mathcal{W}$ is naturally $*$-isomorphic to $\mathcal{M} \bar{\otimes} \mathscr{M}_{N}$, it follows (e.g. from Propositions 2.6.1 and 2.6.2 in [48]) that $\mathcal{W}$ is finite and type I. Further, the results due to Murray and von Neumann [36] (consult also 34] or [40]) imply that the intersection of the domains of all $T_{j k}^{(p)}$ is dense in $\mathcal{H}$, from 
which we infer that each of $S_{p}$ is densely defined. Now if we put $\tilde{T}_{j k}^{(p)} \stackrel{\text { def }}{=}\left(T_{k j}^{(p)}\right)^{*}$, the above argument proves that each of the operators $\tilde{S}_{p} \stackrel{\text { def }}{=}\left[\tilde{T}_{j k}^{(p)}\right]$ is also densely defined. But, a straightforward calculation shows that $\tilde{S}_{p}$ is contained in the adjoint of $S_{p}$, which implies that $S_{p}$ is closable. Denote by $\bar{S}_{p}$ its closure. To conclude that $\left(\bar{S}_{1}, \ldots, \bar{S}_{\ell}\right)$ is FTI, it is sufficient (thanks to Lemma 3.2) that $U S_{p} U^{-1}=S_{p}$ for any unitary operator $U \in \mathcal{W}^{\prime}$. But each such an operator $U$ is of the form $\left[V_{j k}\right]$ where $V_{j k}=0$ for $k \neq j$ and $V_{j j}=V$ for any $j$ where $V$ is a unitary operator in $\mathcal{M}^{\prime}$. Then, since all $T_{j k}^{(p)}$ are affiliated with $\mathcal{M}$, we see that $V T_{j k}^{(p)} V^{-1}=T_{j k}^{(p)}$, from which one readily deduces that $U S_{p} U^{-1}=S_{p}$, and we are done.

\section{REFERENCES}

[1] R.G. Bartle, A general bilinear vector integral, Studia Math. 15 (1956), 337-352.

[2] H. Becker and A.S. Kechris, The Descriptive Set Theory of Polish Group Actions (London Math. Soc. Lecture Note Series, vol. 232), University Press, Cambridge, 1996.

[3] B. Blackadar, Operator Algebras. Theory of $C^{*}$-algebras and von Neumann algebras (Encyclopaedia of Mathematical Sciences, vol. 122: Operator Algebras and Non-Commutative Geometry III), Springer-Verlag, Berlin-Heidelberg, 2006.

[4] A. Brown, The unitary equivalence of binormal operators, Amer. J. Math. 76 (1954), 414-434.

[5] A. Brown, C.-K. Fong, D.W. Hadwin, Parts of operators on Hilbert space, Illinois J. Math. 22 (1978), 306-314.

[6] J.W. Bunce and J.A. Deddens, Irreducible representations of the $C^{*}$-algebra generated by an n-normal operator, Trans. Amer. Math. Soc. 171 (1972), 301-307.

[7] C. Castaing, Quelques problèmes de mesurabilité liées à la théorie de la commande, C. R. Paris 262 (1966), 409-411.

[8] J.B. Conway, A Course in Functional Analysis (Graduate Texts in Mathematics, vol. 96), Springer, New York, 1990.

[9] J. Diestel and J.J. Uhl Jr., Vector Measures, Amer. Math. Soc., Providence, 1977.

[10] N. Dinculeanu, Vector Measures, Pergamon Press Ltd., Oxford, 1967.

[11] J. Dixmier, $C^{*}$-algebras, North-Holland Publ. Co., Amsterdam, 1977.

[12] N. Dunford and J.T. Schwartz, Linear Operators. Part I: General Theory, Interscience Publishers, New York, 1958.

[13] E.G. Effros, The Borel space of von Neumann algebras on a separable Hilbert space, Pacific J. Math. 15 (1965), 1153-1164.

[14] E.G. Effros, Global structure in von Neumann algebras, Trans. Amer. Math. Soc. 121 (1966), $434-454$.

[15] J. Ernest, Charting the operator terrain, Mem. Amer. Math. Soc. 171 (1976), 207 pp.

[16] J.M.G. Fell, The structure of algebras of operator fields, Acta Math. 106 (1961), 233-280.

[17] J. Glimm, A Stone-Weierstrass theorem for $C^{*}$-algebras, Ann. Math. 72 (1960), 216-244.

[18] R.K. Goodrich, A Riesz representation theorem in the setting of locally convex spaces, Trans. Amer. Math. Soc. 131 (1968), 246-258.

[19] R.K. Goodrich, A Riesz representation theorem, Proc. Amer. Math. Soc. 24 (1970), 629-636.

[20] H. Gonshor, Spectral theory for a class of non-normal operators, Canad. J. Math. 8 (1956), 449-461.

[21] D.W. Hadwin, An operator-valued spectrum, Notices Amer. Math. Soc. 23 (1976), A-163.

[22] D.W. Hadwin, An operator-valued spectrum, Indiana Univ. Math. J. 26 (1977), 329-340.

[23] D.W. Hadwin, Continuous functions of operators: a functional calculus, Indiana Univ. Math. J. 27 (1978), 113-125.

[24] D.W. Hadwin, An asymptotic double commutant theorem for $C^{*}$-algebras, Trans. Amer. Math. Soc. 244 (1978), 273-297.

[25] R.V. Kadison and J.R. Ringrose, Fundamentals of the Theory of Operator Algebras. Volume I: Elementary Theory, Academic Press, Inc., New York-London, 1983.

[26] R.V. Kadison and J.R. Ringrose, Fundamentals of the Theory of Operator Algebras. Volume II: Advanced Theory, Academic Press, Inc., Orlando-London, 1986.

[27] D.S. Kaliuzhnyi-Verbovetskyi and V. Vinnikov, Foundations of noncommutative function theory, preprint, arXiv:1212.6345.

[28] I. Kaplansky, A theorem on rings of operators, Pacific J. Math. 1 (1951), 227-232.

[29] J.S. Kim, Ch.R. Kim, S.G. Lee, Reducing operator valued spectra of a Hilbert space operator, J. Korean Math. Soc. 17 (1980), 123-129. 
[30] K. Kuratowski and A. Mostowski, Set Theory with an Introduction to Descriptive Set Theory, PWN - Polish Scientific Publishers, Warszawa, 1976.

[31] K. Kuratowski and C. Ryll-Nardzewski, A general theorem on selectors, Bull. Acad. Pol. Sci. Sér. Sci. Math. 13 (1965), 397-403.

[32] S.G. Lee, Remarks on reducing operator valued spectrum, J. Korean Math. Soc. 16 (1980), $131-136$.

[33] D.R. Lewis, Integration with respect to vector measures, Pacific J. Math. 33 (1970), 157-165.

[34] Z. Liu, On some mathematical aspects of the Heisenberg relation, Sci. China 54 (2011), $2427-2452$.

[35] R. Longo, Solution of the factorial Stone-Weierstrass conjecture. An application of the theory of standard split $W^{*}$-inclusions, Invent. Math. 76 (1984), 145-155.

[36] F. Murray and J. von Neumann, On rings of operators, Ann. Math. 37 (1936), 116-229.

[37] P. Niemiec, Unitary equivalence and decompositions of finite systems of closed densely defined operators in Hilbert spaces, Dissertationes Math. (Rozprawy Mat.) 482 (2012), 1-106.

[38] P. Niemiec, Elementary approach to homogeneous $C^{*}$-algebras, submitted, arXiv:1203.0857.

[39] P. Niemiec, Direct integrals of matrices, submitted, arXiv:1308.2510.

[40] P. Niemiec and A. Wegert, Algebra of operators affiliated with a finite type I von Neumann algebra, submitted, arXiv:1307.4696.

[41] P. Niemiec, Bounded convergence theorems, submitted, arXiv:1309.2612.

[42] P. Niemiec, Models for subhomogeneous $C^{*}$-algebras, submitted, arXiv:????.????.

[43] C. Pearcy, A complete set of unitary invariants for operators generating finite $W^{*}$-algebras of type I, Pacific J. Math. 12 (1962), 1405-1416.

[44] B.J. Pettis, On integration in vector spaces, Trans. Amer. Math. Soc. 44 (1938), $277-304$.

[45] S. Popa, Semiregular maximal abelian *-subalgebras and the solution to the factor state Stone-Weierstrass problem, Invent. Math. 76 (1984), 157-161.

[46] T. Quinn, Factorisation of n-normal operators: products of positive operators, Proc. Royal Irish Acad. 95A (1995), 89-108.

[47] H. Radjavi, On unitary equivalence of arbitrary matrices, Trans. Amer. Math. Soc. 104 (1962), 363-373.

[48] S. Sakai, $C^{*}$-Algebras and $W^{*}$-Algebras, Springer-Verlag, Berlin, 1971.

[49] W.V. Smith and D.H. Tucker, Weak integral convergence theorems and operator measures, Pacific J. Math. 111 (1984), 243-256.

[50] M. Takesaki, Theory of Operator Algebras I (Encyclopaedia of Mathematical Sciences, Volume 124), Springer-Verlag, Berlin-Heidelberg-New York, 2002.

[51] M. Takesaki, Theory of Operator Algebras II (Encyclopaedia of Mathematical Sciences, Volume 125), Springer-Verlag, Berlin-Heidelberg-New York, 2003.

[52] D.H. Tucker and S.G. Wayment, Absolute continuity and the Radon-Nikodym theorem, J. Reine Angew. Math. 244 (1970), 1-19.

[53] J. von Neumann, On Rings of Operators. Reduction Theory, Ann. Math. 50 (1949), 401-485.

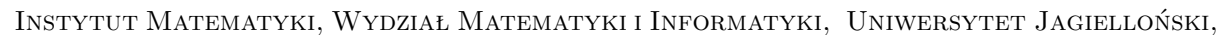
Ul. LojAsiewicza 6, 30-348 Kraków, Poland

E-mail address: piotr.niemiec@uj.edu.pl 